\title{
CTCF chromatin residence time controls 3D genome organization, gene expression and DNA methylation in pluripotent cells
}

Widia Soochit ${ }^{1,8}$, Frank Sleutels ${ }^{1,8}$, Gregoire Stik ${ }^{2,8}$, Marek Bartkuhn ${ }^{3}$, Sreya Basu ${ }^{1}$, Silvia C. Hernandez $^{1}$, Sarra Merzouk ${ }^{4}$, Enrique Vidal ${ }^{2}$, Ruben Boers ${ }^{4}$, Joachim Boers ${ }^{4}$, Michael van der Reijden ${ }^{1}$, Bart Geverts ${ }^{5}$, Wiggert A. van Cappellen ${ }^{5}$, Mirjam van den Hout ${ }^{1,6}$, Zeliha Ozgur $^{1,6}$, Wilfred F. J. van IJcken ${ }^{1,6}$, Joost Gribnau ${ }^{4}$, Rainer Renkawitz ${ }^{3}$, Thomas Graf ${ }^{2}$,

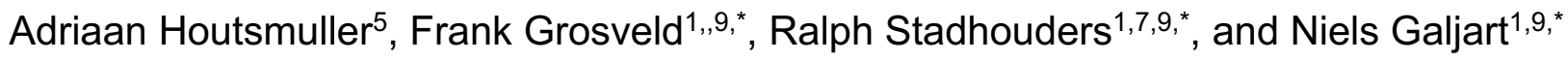

1: Department of Cell Biology, Erasmus MC, University Medical Center, Rotterdam, The Netherlands;

2: Centre for Genomic Regulation (CRG), The Barcelona Institute of Science and Technology (BIST), Barcelona 08003, Spain;

3: Institute for Genetics, Justus-Liebig-University Giessen, Heinrich-Buff-Ring 58-62, 35392, Giessen, Germany;

4: Department of Developmental Biology; Erasmus MC, University Medical Center, Rotterdam, The Netherlands;

5: Department of Pathology, Josephine Nefkens Institute, Erasmus MC, University Medical Center, Rotterdam, The Netherlands;

6: Center for Biomics, Erasmus MC, University Medical Center, The Netherlands;

7: Department of Pulmonary Medicine, Erasmus MC, University Medical Center, Rotterdam, The Netherlands.

8: These authors contributed equally to this work.

9. Co-senior authors.

*: Corresponding authors:

f.grosveld@erasmusmc.nl

r.stadhouders@erasmusmc.nl

n.galjart@erasmusmc.nl 


\begin{abstract}
The eleven zinc finger (ZF) protein CTCF regulates topologically associating domain (TAD) formation and transcription through selective binding to thousands of genomic sites. We replaced endogenous CTCF in mouse embryonic stem cells with GFP-tagged wildtype or mutant proteins lacking individual ZFs to identify additional determinants of CTCF positioning and function. While ZF1 and ZF8-11 are not essential for cell survival, ZF8 deletion strikingly increases the DNA binding off-rate of mutant CTCF, resulting in reduced CTCF chromatin residence time. Loss of ZF8 results in widespread weakening of TADs, aberrant gene expression and increased genome-wide DNA methylation. Thus, important chromatintemplated processes rely on accurate CTCF chromatin residence time, which we propose depends on local sequence and chromatin context as well as global CTCF protein concentration.
\end{abstract}


Nuclear genomes are folded in three dimensions (3D) in a temporally controlled manner that facilitates essential chromatin-templated processes such as transcription, DNA repair, and replication ${ }^{1-3}$. Chromosomal regions segregate into transcriptionally active euchromatic (A) and inactive heterochromatic $(B)$ compartments ${ }^{4,5}$. Inside compartments, there are submegabase sized regions called topologically associating domains $(T A D s)^{6-8}$. Genomic regions inside the same TAD show increased interaction frequencies, creating spatially insulated neighborhoods in the genome. Both compartments and TADs play a role in transcriptional control ${ }^{3}$, with TADs attracting significant attention as a major contributor to the specificity of promoter-enhancer interactions ${ }^{9}$. Notably, disrupted TAD organization has been implicated in developmental disorders and cancer ${ }^{10,11}$.

CTCF is a highly conserved multifunctional eleven zinc finger (ZF) protein that binds thousands of uniquely oriented sites in the genome, each containing a core (C) motif of $\sim 15$ nucleotides $^{12,13}$. Structural analysis of CTCF bound to such a motif revealed that ZFs 3-7 are positioned in the major groove, where each ZF contacts a few bases ${ }^{14}$. By contrast, ZF8 is positioned in the minor groove and does not contribute to binding specificity ${ }^{14}$. Interestingly, a subset of genomic CTCF sites have a small upstream $(U)$ motif, which is separated from the core by a spacer of 5-6 nucleotides ${ }^{12,13,15}$. This extended ' $U C$ ' motif is conserved across species and was proposed to represent a high affinity binding site for CTCF ${ }^{13}$. ZFs 9-11 were shown to bind the $U$ motif $^{15}$, and hence ZF8 is thought to act as a linker between ZFs 4-7 and 9-11, not playing an active role in DNA binding.

Selective binding to its cognate sites in the genome allows CTCF to control gene expression, for example by modulating promoter-enhancer interactions ${ }^{16}$. CTCF also regulates TAD formation together with the cohesin complex ${ }^{17-19}$ in a process termed loop extrusion ${ }^{20}$, where the ring-shaped cohesin complex attaches to chromatin strands either pseudo-topologically or non-topologically ${ }^{21}$ to actively extrude these and generate a loop. Loop dimensions are determined by convergently-oriented CTCF sites ${ }^{5,22,23}$, which block cohesin, allowing it to form a TAD or loop-anchor. Acute degradation of cohesin proteins erases TADs ${ }^{24-27}$, and removing CTCF dissolves TAD borders and increases interactions across borders ${ }^{28,29}$. Thus, both cohesin and CTCF play critical roles in TAD formation. Recently, RNA-binding regions (RBRs) covering ZF1 and a region C-terminal to ZF11 were shown to be involved in CTCF function, also in the context of 3D genome organization ${ }^{30,31}$.

CTCF is required for cell proliferation ${ }^{32,33}$ and is essential in vivo ${ }^{34}$. CTCF depletion studies are therefore limited in that they can only document acute effects after CTCF removal in mitotic cells $\mathbf{s}^{28,29}$ or effects in non-dividing cells ${ }^{35}$. Hence, it remains unclear how CTCF 
controls 3D genome organization and transcription in dividing cells and whether binding specificity is the only determinant of CTCF function. To address these issues, we replaced endogenous CTCF in mouse embryonic stem (ES) cells with fluorescently tagged wild type CTCF or mutant CTCF proteins carrying deletions of individual ZFs. Combining live-cell imaging, biophysics, -omics techniques, and chromosome conformation capture approaches, we show that ZF8 determines CTCF chromatin residence time, which regulates the maintenance of proper 3D genome organization, genome-wide DNA methylation and gene expression in pluripotent cells.

\section{Results}

Generation of embryonic stem cells expressing fluorescently tagged mutant CTCF proteins CTCF contains a central tandem array of eleven ZFs (Extended data Fig.1a). To examine their cellular function we generated cDNAs encoding GFP-tagged wildtype and mutant CTCF with deletions of individual ZFs (Extended data Fig.1b). We cloned the cDNAs together with an IRES-driven Cre into a neomycin resistant lentiviral vector and infected mouse ES cells carrying a floxed Ctcf allele ${ }^{34}$ with the different constructs ${ }^{32}$. As CTCF is essential for ES cells $^{32,33}$, deletion of the floxed Ctcf allele will only give rise to viable ES cells if these are rescued by exogenous CTCF. After lentiviral infection, GFP-positive and neomycin-resistant clones were picked and characterized to confirm individual ZF deletions (data not shown). We did not obtain ES cell clones expressing GFP-CTCF proteins with deletions of ZFs 2-7, indicating that these ZFs are essential for ES cell viability. We did obtain ES cells expressing CTCF mutants lacking ZFs 1 (del1), 8 (del8), 9 (del9), 10 (del10), or 11 (del11) (Fig.1a-b, Extended data Fig.1c). We detected GFP-tagged CTCF, the pluripotency markers Oct4, Sox2 and alkaline phosphatase in all rescued ES cell lines (Fig.1b and data not shown).

Chromatin immunoprecipitation followed by next-generation sequencing (ChIP-Seq) revealed that the binding patterns of wildtype and mutant proteins overlap with published datasets (Fig.1c), demonstrating that the mutant proteins recognize cognate CTCF sites in the mouse genome. Subsequent $k$-means clustering analysis revealed six groups of differentially bound sites, in which most sites were bound less efficiently by one or more of the mutant CTCF proteins (Fig.1d). The del8 mutant displayed the poorest binding to DNA (Extended data Fig.1d). Motif analysis revealed the $U$ motif in sites showing reduced del8del11 binding (Fig.1e). For ZF9-11 the data are consistent with previous reports ${ }^{15,36}$. The fact that ZF8 deletion also revealed this motif indicates that ZF8 indeed acts as a linker for Umotif binding by ZF9-11. Interestingly, we also observed infrequent events of enhanced 
binding by the del8 mutant, revealing the $U$ motif next to the 'core' (Extended data Fig.1e, arrows). These data suggest that ZF9-11 are fully functional in CTCF-del8, but they now contact nucleotides in a nine base-pair region that would normally be the spacer region occupied by ZF8. Finally, in sites where del1 binding was affected we observed appearance of nucleotides downstream of the core motif. Taken together, our data show an extended binding motif for CTCF, consisting of previously described $C$ and $U$ motifs, and a region of weaker consensus on the opposite site of the core, which we term the D (downstream) motif. We note that our D motif differs from a downstream motif identified in an earlier study ${ }^{15}$, both in primary sequence, distance from the core, and function. It does somewhat resemble a module ( "module 4") discovered using the high resolution ChIP-Exo approach ${ }^{12}$. This module was found in approximately $25 \%$ of all CTCF binding sites, with about one third forming an extended UCD site, and two-third forming CD sites. Since there is no endogenous CTCF in the mutant ES cells, no competition can occur between wild type and mutant CTCF. This raises the question why sites with extended motifs, which do contain the core sequence, are less well occupied by mutant CTCF proteins. We hypothesize that these sites are present in regions of the genome where high affinity binding by CTCF is required to counteract strong competitive processes.

Since mutant CTCF proteins displayed selective binding impairments we analyzed the growth and cell cycle properties of the various ES cell lines. While cell proliferation was only slightly reduced in ES cells expressing del9-11, the del1- and del8-expressing ES cells showed significantly slower growth than controls (Extended data Fig.2a). For the del8 mutant, the cell cycle time doubled (Extended data Fig.2a), without impairing a specific phase of the cell cycle (Extended data Fig.2b). Thus, individual deletion of CTCF ZFs 1, 8, 9, 10, or 11 allows pluripotent cell survival, and these ZF mutants represent functional CTCF proteins, albeit with altered DNA binding properties.

\section{Dynamic behaviour of GFP-tagged wild type and mutant CTCF in interphase}

We next examined the intracellular distribution and dynamic behaviour of wild type and mutant GFP-tagged CTCF using various fluorescence-based microscopy approaches. Consistent with other studies in live cells ${ }^{37}$ or in fixed cells using antibodies against CTCF ${ }^{33,38}$, we observed GFP-CTCF in a speckled pattern throughout the interphase nucleus with less signal present in nucleoli, which were readily identified by SiR-Hoechst (Fig.2a, upper panel). While del1 and del9-del11 showed a cellular localization similar to GFP-CTCF (Extended 
data Fig.2c and data not shown), del8 displayed a more diffuse distribution throughout the nucleus and nucleolus (Fig.2a, lower panel).

We define the residence time, or dwell time, of CTCF on DNA as the time that a single CTCF molecule is bound to a cognate site in the genome. The binding frequency of CTCF is defined as the percentage of time a certain site is bound by CTCF molecules. Finally, occupancy of a CTCF site is defined as the efficiency with which a given site is occupied by CTCF in a population of cells. While ChIP-Seq experiments report on CTCF occupancy, fluorescence-based microscopy approaches are required to analyze CTCF residence time and frequency of binding. We first used fluorescence recovery after photobleaching (FRAP), which allows the description of bulk biophysical properties of CTCF. We observed that GFPCTCF was relatively immobile, recovering to $50 \%$ of the initial fluorescence in $\sim 2$ minutes. Initial GFP-CTCF recovery was rapid, but subsequent recovery was slower (Fig.2b), indicating that GFP-CTCF consists of soluble and DNA-bound fractions. Mutants del9, del10, and del11 recovered with similar kinetics, although the DNA-bound fraction in these mutants was somewhat decreased (Fig.2b), in line with our observations that these mutants do not bind UC sites, which form about $10 \%$ of all CTCF sites. In contrast to the del9-del11 mutants, del1 and del8 recovered significantly faster than GFP-CTCF (Fig.2b). The del1 behaviour was not explored in more detail, because it was present at higher levels in ES cells when compared to GFP-CTCF (Extended data Fig.2d). However, del8 was present in similar quantities (Extended data Fig.2d), suggesting that its faster recovery reflects a reduced affinity for DNA.

We applied Monte Carlo simulations to fit the experimentally obtained GFP-CTCF and del8 FRAP curves, using a model that simulates diffusion of molecules and binding of CTCF to immobile elements (representing chromatin) in an ellipsoidal volume (representing the nucleus) $)^{39}$. We obtained relatively good fits for the recoveries of GFP-CTCF and del8 by assuming that these proteins exist in a single conformation and associate with binding sites in the same manner (Fig.2b). These simulations allowed deduction of effective on and offrates, immobile protein fractions and residence (dwell) times (Fig.2c). GFP-CTCF residence time is $\sim 2$ minutes, in line with data obtained using different methodologies ${ }^{37}$. Interestingly, GFP-CTCF and del8 bind with similar on-rates (0.014 versus $0.012 \mathrm{~s}^{-1}$ for GFP-CTCF and del8, respectively) but del8 is released from the DNA with an 8-fold higher off-rate (0.056 versus $0.0071 \mathrm{~s}^{-1}$ for del8 and GFP-CTCF, respectively). Thus, deletion of ZF8 does not affect binding capability but induces a faster release from DNA, leading to higher mobile fractions and a decreased residence time of del8 on DNA as compared to GFP-CTCF 
(Fig.2c). The nuclear localization patterns of GFP-CTCF and del8 (Fig.2a) actually reflect dynamic behaviour. For del8, the mobile fraction is substantially higher than the chromatinbound one (ratio $\sim$, see Fig.2c). We therefore detect mostly freely diffusable del8 protein that traverses the nucleolus. By contrast, GFP-CTCF is largely chromatin-bound (ratio mobile versus immobile fraction of $\sim 0.5$, see Fig.2c) and we therefore mainly detect chromatinbound protein.

Fluorescence correlation spectroscopy (FCS) provides information on fluorescent protein behaviour at a faster temporal scale and with higher sensitivity than FRAP. However, since wild type CTCF protein bound to a cognate site is bleached in the first FCS iteration due to its long residence time on DNA (see online Methods for detailed explanation), we could only use FCS to examine the behaviour of diffuse GFP-CTCF molecules. These measurements revealed a nuclear concentration of $95 \mathrm{nM}$ for GFP-CTCF (Fig.2d). For del8 we found a concentration of $264 \mathrm{nM}$ (Fig.2d), suggesting a 3-fold increase in diffuse del8 compared to GFP-CTCF. These results are in agreement with our modeling data (Fig. 2c) and with an orthogonal approach aimed at measuring the concentrations of nuclear GFPCTCF and del8 by comparing cellular fluorescence intensities to a standard curve of known concentration (Extended data Fig.2e).

To confirm that GFP-CTCF and del8 behaviour are consistent across individual ES cell lines, we investigated one more independently generated GFP-CTCF-expressing clone and two more del8-expressing ES cell clones for cell proliferation, fluorescence localization (i.e diffuse for del8, nucleolus-excluding for GFP-CTCF), and fluorescence intensity as well as recovery after bleaching. We found that all three del8 lines behaved similar and were different from the GFP-CTCF-expressing line in the expected manner (Extended data Fig.3), confirming our earlier observations. Altogether, these experiments suggest that ZF8 mediates stable association of CTCF with chromatin. Importantly, the del8 mutant allows the uncoupling of CTCF binding selectivity from residence time on chromatin.

\section{Imaging of GFP-tagged wild type and mutant CTCF during mitosis}

We next analyzed GFP-CTCF behaviour in mitotic ES cells. We found that fluorescence intensity decreased as cells entered mitosis, with metaphase chromosomes containing the least amount of GFP-CTCF (Fig.3a-b, Extended data Movie 1). After cytokinesis, CTCF was swiftly imported into newly formed daughter nuclei (Fig.3a-b, Extended data Movie 1). This dynamic mitotic behaviour of GFP-CTCF in ES cells is similar to that in HeLa cells, where $\sim 17,000$ CTCF molecules remain DNA-associated in mitosis ${ }^{40}$, an order of magnitude lower 
than the number of CTCF molecules bound to interphase ES cell chromatin ${ }^{41}$. Thus, the number of DNA-bound CTCF molecules is drastically reduced during ES cell mitosis. Interestingly, we observed a specific depletion of GFP-CTCF signal on chromosomal regions with the highest SiR-Hoechst content in prophase cells, indicating that CTCF binding is reduced concomitant with the compaction of mitotic chromatin (Extended data Fig.4).

To compare mitotic and interphase CTCF behaviour, we performed a second set of FRAP experiments. Whereas interphase GFP-CTCF recovered slowly but relatively evenly to $50 \%$ of the initial fluorescence in $\sim 2$ minutes (Fig.3c, see also Fig.2b), metaphase bleaching experiments revealed a rapid initial recovery of GFP-CTCF to approximately $50 \%$ of the pre-bleach value, likely due to the increased numbers of diffuse GFP-CTCF molecules in mitosis, followed by virtual immobility during the remainder of the FRAP experiment (Fig.3c). These results indicate that during mitosis CTCF remains bound to a subset of high affinity binding sites, reminiscent of a role as a mitotic bookmarking factor ${ }^{42}$. Del9, which fails to bind UC sites (see Fig.1), enriched similarly at metaphase chromosomes as compared to GFP-CTCF (Fig.3d,e), indicating that UC sites do not form a significant fraction of high affinity mitotic CTCF sites. By contrast, no del8 enrichment was observed on metaphase chromatin (Fig. 3d,e), suggesting that CTCF binding to mitotic chromosomes is largely controlled by CTCF-DNA interactions.

Impact of reduced CTCF chromatin residence time on genome topology

To examine how the unique behaviour of del8 affects CTCF's role in 3D genome folding and transcriptional regulation, we performed in-situ $\mathrm{Hi}^{-} \mathrm{C}^{5}$ and RNA-sequencing (RNA-Seq) experiments. The latter were performed on the two independently obtained GFP-CTCF lines and the three del8 clones described above. Principal component analysis (PCA) revealed a clear clustering of biological replicate experiments based on the CTCF protein variant expressed, indicating similar transcriptional programs within independent GFP-CTCF or del8 lines (Extended data Fig.5a). In-situ Hi-C experiments were therefore carried out in triplicate but using only one GFP-CTCF and one del8 line. Generated chromatin interaction data showed good quality metrics (Extended data Table 2) and were highly reproducible across triplicates for ES cells expressing GFP-CTCF or del8 (Extended data Fig.5b). No differences in the scaling of contact frequencies as a function of genomic distance were observed in the pooled datasets (Fig.4a, Extended data Fig.5c, Extended data Table 2), indicating that the overall 3D genome conformation is preserved in del8-expressing cells. Likewise, genome segregation into active euchromatic $A$ and inactive heterochromatin $B$ compartments (as 
revealed by PC1 of a principal component analysis of $\mathrm{Hi}-\mathrm{C}$ correlation matrices ${ }^{4}$ ) was similar in GFP-CTCF and del8-expressing cells (Fig.4b,c). Nevertheless, del8-expressing ES cells displayed somewhat weaker segregation of $A$ compartment regions (Fig.4d) and we observed compartment switching across $6 \%$ of the genome in regions of low PC1 score (Fig.4e, Extended data Fig.5d) ${ }^{43}$. RNA-Seq confirmed a modest upregulation of genes in genomic regions that had switched from $B$ to $A$ and the converse for genes that switched from $A$ to $B$ (Extended data Fig.5e). Systematic comparison of overall changes to $A / B$ compartmentalization induced by the ZF8 deletion and those observed after acute CTCF depletion ${ }^{29}$ were very similar (Extended Data Fig.5f,g).

Super-enhancers (SEs) are assemblies of multiple transcription factor binding sites and potent regulators of key cell type-specific gene expression programs ${ }^{44}$, bearing strong resemblance to locus control regions ${ }^{45}$. While individual SEs often reside in different TADs, SEs were shown to cluster into long-range regulatory hubs (Fig.4f $)^{46,47}$, presumably to ensure robust transcriptional activity of target genes ${ }^{3}$. We found that inter-TAD SE clustering was reduced in ES cells expressing del8 (Fig.4g), and that SE-associated genes were more often deregulated than non-SE genes (Fig.4h,i). Consistent with this, expression of several core pluripotency genes was altered in del8-expressing ES cells (Fig.4j). Together, these data indicate that although large-scale genome conformation is maintained in ES cells with weakened CTCF-DNA interactions, loss of high-affinity binding results in changes to $A / B$ compartmentalization, SE clustering and transcriptional regulation of core pluripotency genes.

We next examined genome folding at the level of TADs. Visual inspection of Hi-C maps revealed 'fuzzier' TAD borders in del8-expressing ES cells (Fig.5a). Meta-border plots indeed showed weaker TAD border definitions in del8-expressing cells (Fig.5b), accompanied by a decrease of the inside/outside TAD contact enrichment (Fig.5c). Although border positioning and TAD size was maintained in del8 ES cells (Extended data Fig.6a,b), the ability of del8 to restrict interactions between two adjacent domains (the 'insulation score ${ }^{\prime 48}$ ) was reduced for the majority of TADs. $52 \%$ of TAD borders showed a substantial loss of insulation score ( $>20 \%$ reduction, the 'loss' border category), while only $5 \%$ displayed a robust gain of insulation score ( $>20 \%$ increase, the 'gain' border category) in ES cells expressing del8 (Fig.5d, Extended data Fig.6c). TAD borders affected by the del8 mutation were slightly enriched for $A / B$ compartment junctions and largely overlapped with $T A D$ borders lost after acute CTCF depletion ${ }^{29}$ (Extended data Fig.6d,e). Although the average loss of insulation per border was more pronounced after complete CTCF depletion, ZF8 deletion had a substantial negative impact on TAD border strength (Extended data Fig.6f). 
Interestingly, the 'loss' TAD border category displayed a significantly stronger insulation score (i.e. lower values) in wildtype ES cells than 'stable' or 'gain' borders (Fig.5e). In agreement, borders occupied by CTCF showed more pronounced reductions in insulation score than those not bound by CTCF (Extended data Fig.6g). Borders weakened in del8expressing cells showed the strongest CTCF enrichment in wildtype cells (Fig.5e) but also displayed a significantly larger loss of del8 binding in ChIP-Seq experiments (Fig.5f). Combined, these data show that strong TAD borders depend on sufficient CTCF residence time and occupancy to maintain insulation potential.

The capacity of CTCF to block loop extrusion by cohesin is reflected by the formation and intensity of chromatin loops, which often reside at the apex of TADs in Hi-C maps ${ }^{5}$. De novo loop calling revealed that the del8 mutation reduced loop intensity (Fig.5g), which was confirmed by meta-analysis on a larger set of validated ES cell loops ${ }^{43,49}$ (Extended data Fig.6i). These data indicate that chromatin residence time is important for blocking loop extrusion by CTCF. Nevertheless, TADs that show evidence of loop extrusion (i.e. loop formation) are not preferentially affected, as border strength changes were similar for TADs formed by cohesin-mediated loops ${ }^{50}$ compared to those that are not (Extended data Fig.6h). We conclude that TAD border strength is determined by the number of CTCF molecules actually residing at the border at a given moment in time, which in turn is determined by both the number of binding sites and the residence time, irrespective of whether TADs appear as loop extrusion domains or domains formed by alternative mechanisms (e.g. compartment domains $\left.{ }^{2,51}\right)$.

In female cells, the inactive $X$ chromosome adopts a unique 3D conformation driven by a strong border element at the $D x z 4$ locus, resulting in the formation of two mega-domains 5,52-54. Interestingly, we observed the de novo formation of a large domain on the $X$ chromosome in the female del8-expressing ES cells, which was centered on the Dxz4 locus (Extended data Fig.6j,k). Formation of this mega-domain (covering $\sim 22 \%$ of the $X$ chromosome) was not accompanied by the appearance of novel CTCF binding sites at its borders (Extended data Fig.6k), implicating a CTCF-independent mechanism in forming this structure.

Transcription elongation is linked to impaired chromatin residence time of del8 CTCF CTCF sites at TAD borders tend to retain important nucleotides in the 'core' motif that mediate binding to ZFs 3-7 of CTCF 28,55 . These sites should therefore also be bound relatively well by del8. To understand why this is not the case at the 'lost' border category (33\% overall 
reduction in signal versus $16 \%$ at stable border CTCF peaks, Fig.5h), we investigated the chromatin and transcriptional landscape of these TAD borders in pluripotent cells. Notably, we observed higher transcriptional output at border regions weakened by the del8 mutation (Fig.5i), suggesting that aspects of transcriptional control correlate with the local loss of del8 binding. While genes at borders that lost insulation potential were more often deregulated in del8-expressing ES cells than genes at stable borders (Fig.5j), the combined average transcriptional output from all border categories did not significantly change (Extended data Fig.6I). Chromatin state analysis using publicly available ES cell datasets revealed increased H3K79Me2 and H3K36Me3 levels at CTCF sites within strong borders that lose insulation strength in del8-expressing cells (Fig.5k). These modifications are associated with actively transcribed genes and specifically with elongating RNA polymerase $\|^{56}$. In agreement, H3K27Me3, a histone mark that signals Polycomb-repressed chromatin ${ }^{57}$, was relatively depleted at these borders (Fig.5k). In addition, we found that UC motifs were absent a few hundred bases downstream of the transcription start site (Extended data Fig.6m), suggesting that initiation of transcription elongation is incompatible with high-affinity binding of CTCF, and hence prolonged CTCF residence time on DNA. Combined, these results indicate that transcription elongation and CTCF binding are competitive processes and that actively transcribed chromatin at strong TAD borders negatively influences del8 binding.

Reduced CTCF chromatin residence time results in transcriptional deregulation and widespread DNA methylation defects

We next further investigated the transcriptional consequences of the ZF8 deletion in pluripotent stem cells. Differential gene expression analysis identified 1030 downregulated and 948 upregulated genes in del8-expressing ES cells (Fig.6a, Extended data Table I). These genes showed a relatively small overlap with genes found deregulated 48 hours after acute CTCF depletion ${ }^{29}$, or in ES cells containing an RNA-binding region (RBR) mutant of $\mathrm{CTCF}^{30}$ (Extended data Fig.7a,b). Hence, disrupting CTCF residence time elicits unique transcriptional defects that affects a substantial number of genes.

Among the differentially expressed genes (DEGs) in del8, the downregulated ones more often contained a CTCF site near their promoter (Fig.6b). In addition, in GFP-CTCFexpressing cells these genes displayed substantially higher expression levels than upregulated genes (Fig.6c). These results are in line with transcriptional stimulation by CTCF via promoter occupancy ${ }^{28,29}$. Interestingly, we observed that del8 binding was generally less affected on promoter sites than on non-promoter sites (Fig.6d). In wildtype ES cells promoter 
CTCF sites were nucleosome-depleted, highly accessible and transcriptionally active, while non-promoter sites were embedded in nucleosomal DNA, showed low accessibility and were devoid of active chromatin (Fig.6e). Thus, chromatin accessibility on promoter sites may alleviate the impact of ZF8 deletion on CTCF chromatin occupancy.

Gene ontology enrichment analysis using Metascape ${ }^{58}$ was next used to explore possible biological pathways deregulated in del8-expressing ES cells. Genes affected were involved in metabolic processes, cell adhesion and cellular signaling, suggesting that highaffinity CTCF binding is important for maintaining the pluripotent gene expression program (Extended data Fig.7c) - in line with the deregulation of SE-associated genes (Fig.4). Further inspection of DEGs (Extended data Table I) revealed that one of the upregulated genes in del8-expressing ES cells is the de novo DNA methyltransferase Dnmt3a (Fig.7a). Western blot and immunofluorescence experiments confirmed increased Dnmt3a levels in multiple del8-expressing ES cell lines (Fig.7b, Extended data Fig.8a,b). Furthermore, we also observed increased expression of Dnmt3b in del8-expressing ES cells (Extended data Fig.8c). In line with elevated de novo methyltransferase activity, nuclear 5-methylcytosine $(5 \mathrm{mC})$ levels were increased in del8-expressing ES cells (Extended data Fig.8d). To assess DNA methylation changes at higher resolution we performed MeD-Seq, a recently developed high-throughput method that interrogates $>50 \%$ of all methylated sites in the genome ${ }^{59}$. PCA analysis indicated similar DNA methylation patterns in the three independent del8-expressing ES cell clones analyzed (Extended data Fig.8e). We called differentially methylated regions (DMRs) between wildtype and del8-expressing ES cells using stringent criteria (see Methods) and observed 290 hypermethylated DMRs and 82 hypomethylated DMRs in del8-expressing ES cells (Fig.7c), consistent with elevated Dnmt3a activity. These DMRs localized to promoters, gene bodies and intergenic regions (Extended data Fig.8f). DNA methylation has previously been linked to reduced CTCF binding ${ }^{60-63}$. However, in del8-expressing cells DNA hypermethylation did not correlate with decreased CTCF occupancy (Extended data Fig.8g) and only very few CTCF sites located in weakened TAD borders or that showed reduced occupancy colocalized with hypermethylated DMRs ( 0.5\%, Fig.7d). Thus, ZF8 deletion causes aberrant DNA methylation, presumably through inadvertent activation of de novo methyltransferases, although DNA hypermethylation cannot explain weakened del8 binding.

We next examined CTCF binding at the Dnmt3a locus and observed a selective loss of several CTCF peaks in the del8 mutant, including one near the promoter of the long Dnmt3a isoform (Fig.7e). In addition, the 5' border of the Dnmt3a TAD was weakened in 
del8-expressing ES cells (Fig.7f), and high-resolution analysis within the Dnmt3a locus revealed increased interactions between the promoters of the long and short Dnmt3a isoforms in del8-expressing cells, as well as elevated interactions of the short isoform promoter with multiple nearby $\mathrm{H} 3 \mathrm{~K} 27 \mathrm{Ac}+$ putative regulatory regions (Fig.7g). Together, these data suggest that CTCF chromatin residence time controls Dnmt3a expression by shaping local 3D genome topology.

\section{Discussion}

In this study we engineered pluripotent stem cell lines in which we replaced endogenous CTCF by fluorescently labeled wildtype or ZF mutant CTCF proteins expressed at physiological levels. We focused on the deletion of ZF8, because this particular mutation allows uncoupling of CTCF binding selectivity from its chromatin residence time. While twothird of wildtype GFP-CTCF is DNA-bound, only $20 \%$ of the del 8 mutant protein is DNAbound due to a higher off-rate. Although this results in an increased nuclear concentration which stimulates del8 binding frequency and binding site occupancy, on many binding sites this does not compensate for the substantially lower residence time of del8 on chromatin.

Our FCS experiments suggest that diffuse GFP-CTCF partitions into two nonmonomeric fractions. Approximately $80 \%$ of diffuse CTCF is found in Fraction 1 (F1, see Fig.2e), which could represent high molecular weight CTCF-RNA complexes ${ }^{64,65}$. Of note, the diffusion coefficient D1 of del8 was found to be lower than D1 of GFP-CTCF, suggesting that del8 might be present in even higher molecular weight CTCF-RNA complexes. Fraction 2 of GFP-CTCF molecules (F2, $20 \%$ of CTCF molecules) has a coefficient consistent with a diffusion time of $\sim 130 \mathrm{~ms}$. This value is similar to the value $(\sim 0.2$ seconds) found in single molecule experiments for CTCF molecules binding to chromatin aspecifically and transiently ${ }^{66}$. We did not observe an F2 fraction in our del8 experiments, suggesting that ZF8 is important for transient CTCF-DNA interactions. Interestingly, FRAP experiments using overexpressed CTCF proteins show recovery half lives in the order of seconds ${ }^{15}$, instead of minutes as found by us and others ${ }^{37,65}$. These differences can be explained by assuming that overexpressed fluorescent CTCF is present in large excess and competes with binding for normal CTCF sites with endogenous protein; the fast recovery times would therefore mainly reflect random interactions of overexpressed fluorescent CTCF with DNA. If this assumption is correct, the FRAP results with overexpressed GFP-CTCF suggest that most ZFs contribute to transient DNA interactions ${ }^{15}$. 
In agreement with other studies ${ }^{40,67}$, our imaging data suggest that CTCF remains bound to mitotic chromosomes but only to a small subset of high-affinity binding sites. Results with the del8 mutant, which does not associate with metaphase chromosomes, indicate that the association of CTCF with mitotic chromosomes is largely DNA-mediated. The subset of mitotic high-affinity CTCF binding sites may serve to facilitate rapid re-establishment of genome folding patterns after mitosis in normal ES cells. However, since del8-expressing ES cells do progress through mitosis and establish TADs, we conclude that mitotic CTCF-DNA interactions are not strictly required for mitosis or for TAD reformation after mitosis.

Ample evidence supports the view that most TADs are formed by a cohesin-CTCF interplay. The dynamic loop maintenance complex model ${ }^{37,68}$, which is an extension of the loop extrusion model ${ }^{69}$, proposes that CTCF, with an average residence time of about 2 minutes on cognate sites, acts as a barrier for cohesin-mediated loop extrusion by constantly binding and unbinding at loop-convergent sites. Our data support this model and suggest that partial loss of this barrier function in the del8 mutant results in "fuzzier" TADs due to less efficient trapping of cohesin. TAD borders often contain clusters of CTCF sites ${ }^{55}$. Our finding that insulation strength of such strong borders with multiple CTCF sites depends on an intact ZF8, indicates that while selective CTCF recruitment to convergent sites may determine TAD border positioning, high affinity binding of CTCF to multiple sites in a border is required to maintain TAD border strength.

Inside TADs, CTCF has been shown to regulate interactions between gene regulatory elements and contribute to transcriptional control ${ }^{16,70}$. Our data indicate that lowering CTCF residence time can disrupt local regulatory interactions as illustrated by the Dnmt3a locus. Here, the del8 mutation results in the upregulation of both the long and short isoforms of Dnmt3a. CTCF normally binds the Dnmt3a long isoform promoter, and binding is abrogated upon ZF8 deletion. As a consequence, both Dnmt3a promoters show increased interactions with nearby $\mathrm{H} 3 \mathrm{~K} 27 \mathrm{Ac}+$ putative regulatory elements, explaining the upregulation of both isoforms in del8-expressing cells. Thus, CTCF might restrict intra-TAD interactions of the Dnmt3a promoters with active regulatory regions to ensure appropriate levels of Dnmt3a enzymatic activity in pluripotent cells. In addition, CTCF could also function as a direct transcriptional repressor of the long isoform.

The mechanisms underlying $A / B$ compartmentalization and the formation of compartment domains, a class of TADs whose borders align with $A / B$ compartment transitions ${ }^{51}$, remain poorly understood. ZF8 deletion does not destabilize A/B compartmentalization, even when assessed over many rounds of cell division. However, 
long-range SE clustering at the compartment level is negatively affected by the del8 mutation, indicating that proper CTCF residence time facilitates the maintenance of long-range interTAD interactions. Increased promiscuous local interactions across TAD borders in the del8 mutant may come at the expense of long-range inter-TAD interaction frequencies of SEs, leading to a deregulated transcriptional output of SE-associated genes ${ }^{71}$. Reduced CTCF residence time also allowed for the formation of a novel megadomain on the $\mathrm{X}$ chromosome, with at its center the Dxz4 locus that was shown to form a border that separates the two megadomains of the inactive $X$ chromosome in ES cells ${ }^{52}$. While the significance of this phenomenon is unclear, del8 CTCF does not appear to play a direct role in de novo formation of this megadomain, as CTCF binding was similarly reduced in its border regions.

Uncoupling CTCF binding capacity from residence time revealed chromatin features that modulate CTCF dwell time on DNA. We found, for example, that binding of the del8 mutant was more affected at highly transcribed TAD borders within a chromatin environment associated with transcription elongation. These data suggest that TAD border insulation strength and transcription elongation compete with each other by regulating CTCF chromatin occupancy, in line with a recent study showing that transcription can displace CTCF from chromatin ${ }^{72}$. These findings can also explain the observation that CTCF binding sites at TAD borders are conserved and of relatively high affinity ${ }^{28,55}$, as they need to withstand strong transcription elongation forces ${ }^{73}$. By contrast, comparing CTCF sites at promoter and nonpromoter regions revealed that loss of del8 binding was generally less pronounced at promoters. An open chromatin environment could allow del8, which is present in higher nuclear concentrations than wild type CTCF, to rapidly re-bind after premature dissociation. In agreement, CTCF binding sites near promoters were found to be less conserved with respect to nucleotides that mediate CTCF-DNA major groove interactions ${ }^{31}$, suggesting that an open chromatin environment is compatible with a somewhat lower affinity of CTCF binding. However, the rapid re-binding of del8 at promoter sites is not enough to prevent downregulation of expression of genes with del8 bound to their promoters, raising the interesting possibility that a proper CTCF residence time at promoter sites is required for efficient transcription initiation.

Several recent studies have added important nuance to the role of CTCF and TADs in transcriptional control, revealing that CTCF-mediated genome topology plays a contextspecific instead of pleiotropic role in regulating gene expression ${ }^{24-26,29,70}$. Our findings corroborate this concept, and also extend it by demonstrating that impairing CTCF function across several cell divisions affects cell type-specific transcriptional programs. While 
compatible with ES cell survival and cell division, reduced CTCF chromatin residence time results in lower proliferation rates and transcriptional defects, including the deregulation of SE-associated genes and key regulators of DNA methylation. In this respect, the effects of ZF8 deletion on the gene expression program of pluripotent cells are largely unique, as compared to acute CTCF depletion ${ }^{29}$ or recently described CTCF mutants defective in RNA binding ${ }^{30,31}$. One explanation for this phenomenon is that reduced CTCF residence time affects a distinct set of CTCF binding sites, which in turn leads to unique transcriptional changes. Additionally, the de novo DNA methylation observed in del8-expressing cells due to aberrant upregulation of $D n m t 3 a$ likely further augments gene expression defects ${ }^{74}$. Hence, optimal CTCF residence time is required for maintaining the transcriptional and phenotypic identity of pluripotent stem cells. Somatic CTCF mutations in human cancer genomes have been reported before ${ }^{75}$ and were recently mapped in great detail ${ }^{76}$. Missense mutations in the broader ZF domain were shown to occur more frequently than expected, suggesting that the $\mathrm{ZF}$ region is frequently altered in cancer. Missense mutations were also observed in $\mathrm{ZF8}^{76}$, indicating that this ZF contributes to CTCF function in vivo.

In conclusion, we have shown that chromatin residence time is an important parameter of CTCF binding and function. Based on our data and on structural studies of $\mathrm{CTCF}^{14}$, we propose that at core-only (C) sites ZF8 functions as a clamp that clasps CTCF to DNA (Fig.7h), thereby increasing residence time. In contrast, on UC sites ZF8 acts as a linker ${ }^{15}$, bridging the $U$ and $C$ motifs, which together form a high affinity binding site that increases CTCF residence time (Fig.7h). The dual role of ZF8 as clamp or linker explains the selective loss of del8 from UC sites and its decreased binding efficiency on core-only sites. Our ChIPSeq data furthermore indicate that ZF1 participates in binding to $D$ sites downstream of the core motif (Fig.7h). On UCD sites CTCF is fully stretched out on DNA which might have consequences for cohesin-blocking. Of note, as ZF1 was also shown to interact with RNA ${ }^{31}$, it may also have a dual role as an RNA- and DNA-binding module of CTCF. CTCF residence time is locally further determined by chromatin environment and transcription elongation in addition to sequence context, and globally by the concentration of diffusible CTCF that is continuously 'absorbed' by competing CTCF binding sites (Fig.7h). Functionally, a proper CTCF residence time enables pluripotent stem cells to i) maintain proper TAD organization and SE clustering, ii) restrict local interactions between regulatory elements, iii) oppose aberrant DNA methylation and iv) sustain a normal gene expression program. 


\section{Acknowledgements}

We would like to thank Isabella Zampeta for performing the Dnmt3a western blots, and Reinier van der Linden, Rick Janssens, and Marjolein de Bruijn for performing flow cytometry. This work was supported by grants from the Netherlands Organisation for Scientific Research (ALW 822.02.018) and the Dutch Cancer Society (KWF EMCR 2008-4109). G.S. was supported by a Marie Skłodowska-Curie fellowship (H2020-MSCA-IF-2016, miRStem) and by the 'Fundación Científica de la Asociación Española Contra el Cáncer'. R.S. is supported by the Netherlands Organization for Scientific Research (VENI 91617114) and an Erasmus MC Fellowship.

\section{Author Contributions}

WS, FS, and MvdR generated and characterized the GFP-tagged ES cell lines; WS, FS, MvdH, ZO, WFJvIJ, SB, SCH, RS, NG, and MB performed and analyzed RNA-Seq and ChIPSeq experiments; $\mathrm{SCH}, \mathrm{SB}, \mathrm{BG}, \mathrm{WavC}, \mathrm{AH}$, and $\mathrm{NG}$ performed and analyzed imaging experiments; SB, GS, EV, and RS performed and analyzed $\mathrm{HiC}$ experiments; SB, RB, JB, JG, NG, and RS performed and analyzed DNA methylation experiments; SB, SM, JG, RS, and NG performed and analyzed FISH experiments; RR, TG, AH, FG, RS, and NG conceived many of the experiments presented in this work and analyzed results; all authors contributed to the writing of this manuscript.

\section{Competing Interests statement.}

R.B., J.B., W.V.I. and J.G. report being shareholder in Methylomics B.V., a commercial company that applies MeD-seq to develop methylation markers for cancer staging. The other authors declare no conflict of interest or financial interests.

\section{Data Availability.}

The datasets generated during the current study are available in the GEO data repository under the GEO Accession number GSE154009. All data generated during this study are included in this published article (and its supplementary information files). 


\section{References}

1. Bonev, B. \& Cavalli, G. Organization and function of the 3D genome. Nat Rev Genet 17, 772 (2016).

2. Rowley, M.J. \& Corces, V.G. Organizational principles of 3D genome architecture. Nat Rev Genet 19, 789-800 (2018).

3. Stadhouders, R., Filion, G.J. \& Graf, T. Transcription factors and 3D genome conformation in cell-fate decisions. Nature 569, 345-354 (2019).

4. Lieberman-Aiden, E., van Berkum, N.L., Williams, L., Imakaev, M., Ragoczy, T., Telling, A., Amit, I., Lajoie, B.R., Sabo, P.J., Dorschner, M.O., Sandstrom, R., Bernstein, B., Bender, M.A., Groudine, M., Gnirke, A., Stamatoyannopoulos, J., Mirny, L.A., Lander, E.S. \& Dekker, J. Comprehensive mapping of long-range interactions reveals folding principles of the human genome. Science 326, 289-93 (2009).

5. Rao, S.S., Huntley, M.H., Durand, N.C., Stamenova, E.K., Bochkov, I.D., Robinson, J.T., Sanborn, A.L., Machol, I., Omer, A.D., Lander, E.S. \& Aiden, E.L. A 3D map of the human genome at kilobase resolution reveals principles of chromatin looping. Cell 159, 1665-80 (2014).

6. Dixon, J.R., Selvaraj, S., Yue, F., Kim, A., Li, Y., Shen, Y., Hu, M., Liu, J.S. \& Ren, B. Topological domains in mammalian genomes identified by analysis of chromatin interactions. Nature 485, 376-80 (2012).

7. Nora, E.P., Lajoie, B.R., Schulz, E.G., Giorgetti, L., Okamoto, I., Servant, N., Piolot, T., van Berkum, N.L., Meisig, J., Sedat, J., Gribnau, J., Barillot, E., Bluthgen, N., Dekker, J. \& Heard, E. Spatial partitioning of the regulatory landscape of the X-inactivation centre. Nature 485, 381-5 (2012).

8. Sexton, T., Yaffe, E., Kenigsberg, E., Bantignies, F., Leblanc, B., Hoichman, M., Parrinello, H., Tanay, A. \& Cavalli, G. Three-dimensional folding and functional organization principles of the Drosophila genome. Cell 148, 458-72 (2012).

9. Schoenfelder, S. \& Fraser, P. Long-range enhancer-promoter contacts in gene expression control. Nat Rev Genet 20, 437-455 (2019).

10. Groschel, S., Sanders, M.A., Hoogenboezem, R., de Wit, E., Bouwman, B.A.M., Erpelinck, C., van der Velden, V.H.J., Havermans, M., Avellino, R., van Lom, K., Rombouts, E.J., van Duin, M., Dohner, K., Beverloo, H.B., Bradner, J.E., Dohner, H., Lowenberg, B., Valk, P.J.M., Bindels, E.M.J., de Laat, W. \& Delwel, R. A single oncogenic enhancer rearrangement causes concomitant EVI1 and GATA2 deregulation in leukemia. Cell 157, 369-381 (2014).

11. Lupianez, D.G., Kraft, K., Heinrich, V., Krawitz, P., Brancati, F., Klopocki, E., Horn, D., Kayserili, H., Opitz, J.M., Laxova, R., Santos-Simarro, F., Gilbert-Dussardier, B., Wittler, L., Borschiwer, M., Haas, S.A., Osterwalder, M., Franke, M., Timmermann, B., Hecht, J., Spielmann, M., Visel, A. \& Mundlos, S. Disruptions of topological chromatin domains cause pathogenic rewiring of gene-enhancer interactions. Cell 161, 1012-1025 (2015).

12. Rhee, H.S. \& Pugh, B.F. Comprehensive genome-wide protein-DNA interactions detected at singlenucleotide resolution. Cell 147, 1408-19 (2011). 
13. Schmidt, D., Schwalie, P.C., Wilson, M.D., Ballester, B., Goncalves, A., Kutter, C., Brown, G.D., Marshall, A., Flicek, P. \& Odom, D.T. Waves of retrotransposon expansion remodel genome organization and CTCF binding in multiple mammalian lineages. Cell 148, 335-48 (2012).

14. Hashimoto, H., Wang, D., Horton, J.R., Zhang, X., Corces, V.G. \& Cheng, X. Structural Basis for the Versatile and Methylation-Dependent Binding of CTCF to DNA. Mol Cell 66, 711-720 e3 (2017).

15. Nakahashi, H., Kwon, K.R., Resch, W., Vian, L., Dose, M., Stavreva, D., Hakim, O., Pruett, N., Nelson, S., Yamane, A., Qian, J., Dubois, W., Welsh, S., Phair, R.D., Pugh, B.F., Lobanenkov, V., Hager, G.L. \& Casellas, R. A genome-wide map of CTCF multivalency redefines the CTCF code. Cell Rep 3, 1678-89 (2013).

16. Holwerda, S.J. \& de Laat, W. CTCF: the protein, the binding partners, the binding sites and their chromatin loops. Philos Trans R Soc Lond B Biol Sci 368, 20120369 (2013).

17. Dekker, J. \& Mirny, L. The 3D Genome as Moderator of Chromosomal Communication. Cell 164, 1110-1121 (2016).

18. Merkenschlager, M. \& Nora, E.P. CTCF and Cohesin in Genome Folding and Transcriptional Gene Regulation. Annu Rev Genomics Hum Genet 17, 17-43 (2016).

19. Sanborn, A.L., Rao, S.S., Huang, S.C., Durand, N.C., Huntley, M.H., Jewett, A.I., Bochkov, I.D., Chinnappan, D., Cutkosky, A., Li, J., Geeting, K.P., Gnirke, A., Melnikov, A., McKenna, D., Stamenova, E.K., Lander, E.S. \& Aiden, E.L. Chromatin extrusion explains key features of loop and domain formation in wild-type and engineered genomes. Proc Natl Acad Sci U S A 112, E6456-65 (2015).

20. Fudenberg, G., Abdennur, N., Imakaev, M., Goloborodko, A. \& Mirny, L.A. Emerging Evidence of Chromosome Folding by Loop Extrusion. Cold Spring Harb Symp Quant Biol 82, 45-55 (2017).

21. Davidson, I.F., Bauer, B., Goetz, D., Tang, W., Wutz, G. \& Peters, J.M. DNA loop extrusion by human cohesin. Science 366, 1338-1345 (2019).

22. Gomez-Marin, C., Tena, J.J., Acemel, R.D., Lopez-Mayorga, M., Naranjo, S., de la Calle-Mustienes, E., Maeso, I., Beccari, L., Aneas, I., Vielmas, E., Bovolenta, P., Nobrega, M.A., Carvajal, J. \& GomezSkarmeta, J.L. Evolutionary comparison reveals that diverging CTCF sites are signatures of ancestral topological associating domains borders. Proc Natl Acad Sci U S A 112, $7542-7$ (2015).

23. Vietri Rudan, M., Barrington, C., Henderson, S., Ernst, C., Odom, D.T., Tanay, A. \& Hadjur, S. Comparative Hi-C reveals that CTCF underlies evolution of chromosomal domain architecture. Cell Rep 10, 1297-309 (2015).

24. Haarhuis, J.H.I., van der Weide, R.H., Blomen, V.A., Yanez-Cuna, J.O., Amendola, M., van Ruiten, M.S., Krijger, P.H.L., Teunissen, H., Medema, R.H., van Steensel, B., Brummelkamp, T.R., de Wit, E. \& Rowland, B.D. The Cohesin Release Factor WAPL Restricts Chromatin Loop Extension. Cell 169, 693-707 e14 (2017).

25. Rao, S.S.P., Huang, S.C., Glenn St Hilaire, B., Engreitz, J.M., Perez, E.M., Kieffer-Kwon, K.R., Sanborn, A.L., Johnstone, S.E., Bascom, G.D., Bochkov, I.D., Huang, X., Shamim, M.S., Shin, J., Turner, D., Ye, Z., Omer, A.D., Robinson, J.T., Schlick, T., Bernstein, B.E., Casellas, R., Lander, E.S. \& Aiden, E.L. Cohesin Loss Eliminates All Loop Domains. Cell 171, 305-320 e24 (2017). 
26. Schwarzer, W., Abdennur, N., Goloborodko, A., Pekowska, A., Fudenberg, G., Loe-Mie, Y., Fonseca, N.A., Huber, W., C, H.H., Mirny, L. \& Spitz, F. Two independent modes of chromatin organization revealed by cohesin removal. Nature 551, 51-56 (2017).

27. Wutz, G., Varnai, C., Nagasaka, K., Cisneros, D.A., Stocsits, R.R., Tang, W., Schoenfelder, S., Jessberger, G., Muhar, M., Hossain, M.J., Walther, N., Koch, B., Kueblbeck, M., Ellenberg, J., Zuber, J., Fraser, P. \& Peters, J.M. Topologically associating domains and chromatin loops depend on cohesin and are regulated by CTCF, WAPL, and PDS5 proteins. EMBO J 36, 3573-3599 (2017).

28. Zuin, J., Dixon, J.R., van der Reijden, M.I., Ye, Z., Kolovos, P., Brouwer, R.W., van de Corput, M.P., van de Werken, H.J., Knoch, T.A., van, I.W.F., Grosveld, F.G., Ren, B. \& Wendt, K.S. Cohesin and CTCF differentially affect chromatin architecture and gene expression in human cells. Proc Natl Acad Sci U S A 111, 996-1001 (2014).

29. Nora, E.P., Goloborodko, A., Valton, A.L., Gibcus, J.H., Uebersohn, A., Abdennur, N., Dekker, J., Mirny, L.A. \& Bruneau, B.G. Targeted Degradation of CTCF Decouples Local Insulation of Chromosome Domains from Genomic Compartmentalization. Cell 169, 930-944 e22 (2017).

30. Hansen, A.S., Hsieh, T.S., Cattoglio, C., Pustova, I., Saldana-Meyer, R., Reinberg, D., Darzacq, X. \& Tjian, R. Distinct Classes of Chromatin Loops Revealed by Deletion of an RNA-Binding Region in CTCF. Mol Cell (2019).

31. Saldana-Meyer, R., Rodriguez-Hernaez, J., Escobar, T., Nishana, M., Jacome-Lopez, K., Nora, E.P., Bruneau, B.G., Tsirigos, A., Furlan-Magaril, M., Skok, J. \& Reinberg, D. RNA Interactions Are Essential for CTCF-Mediated Genome Organization. Mol Cell (2019).

32. Sleutels, F., Soochit, W., Bartkuhn, M., Heath, H., Dienstbach, S., Bergmaier, P., Franke, V., RosaGarrido, M., van de Nobelen, S., Caesar, L., van der Reijden, M., Bryne, J.C., van Ijcken, W., Grootegoed, J.A., Delgado, M.D., Lenhard, B., Renkawitz, R., Grosveld, F. \& Galjart, N. The male germ cell gene regulator CTCFL is functionally different from CTCF and binds CTCF-like consensus sites in a nucleosome composition-dependent manner. Epigenetics Chromatin 5, 8 (2012).

33. van de Nobelen, S., Rosa-Garrido, M., Leers, J., Heath, H., Soochit, W., Joosen, L., Jonkers, I., Demmers, J., van der Reijden, M., Torrano, V., Grosveld, F., Delgado, M.D., Renkawitz, R., Galjart, N. \& Sleutels, F. CTCF regulates the local epigenetic state of ribosomal DNA repeats. Epigenetics Chromatin 3, 19 (2010).

34. Heath, H., Ribeiro de Almeida, C., Sleutels, F., Dingjan, G., van de Nobelen, S., Jonkers, I., Ling, K.W., Gribnau, J., Renkawitz, R., Grosveld, F., Hendriks, R.W. \& Galjart, N. CTCF regulates cell cycle progression of alphabeta T cells in the thymus. Embo J 27, 2839-50 (2008).

35. Stik, G., Vidal, E., Barrero, M., Cuartero, S., Vila-Casadesus, M., Mendieta-Esteban, J., Tian, T.V., Choi, J., Berenguer, C., Abad, A., Borsari, B., le Dily, F., Cramer, P., Marti-Renom, M.A., Stadhouders, R. \& Graf, T. CTCF is dispensable for immune cell transdifferentiation but facilitates an acute inflammatory response. Nat Genet (2020).

36. Vian, L., Pekowska, A., Rao, S.S.P., Kieffer-Kwon, K.R., Jung, S., Baranello, L., Huang, S.C., El Khattabi, L., Dose, M., Pruett, N., Sanborn, A.L., Canela, A., Maman, Y., Oksanen, A., Resch, W., Li, X., Lee, B., Kovalchuk, A.L., Tang, Z., Nelson, S., Di Pierro, M., Cheng, R.R., Machol, I., St Hilaire, B.G., Durand, N.C., Shamim, M.S., Stamenova, E.K., Onuchic, J.N., Ruan, Y., Nussenzweig, A., 
Levens, D., Aiden, E.L. \& Casellas, R. The Energetics and Physiological Impact of Cohesin Extrusion. Cell 173, 1165-1178 e20 (2018).

37. Hansen, A.S., Pustova, I., Cattoglio, C., Tjian, R. \& Darzacq, X. CTCF and cohesin regulate chromatin loop stability with distinct dynamics. Elife 6(2017).

38. Burke, L.J., Zhang, R., Bartkuhn, M., Tiwari, V.K., Tavoosidana, G., Kurukuti, S., Weth, C., Leers, J., Galjart, N., Ohlsson, R. \& Renkawitz, R. CTCF binding and higher order chromatin structure of the H19 locus are maintained in mitotic chromatin. Embo J 24, 3291-300 (2005).

39. Geverts, B., van Royen, M.E. \& Houtsmuller, A.B. Analysis of biomolecular dynamics by FRAP and computer simulation. Methods Mol Biol 1251, 109-33 (2015).

40. Cai, Y., Hossain, M.J., Heriche, J.K., Politi, A.Z., Walther, N., Koch, B., Wachsmuth, M., Nijmeijer, B., Kueblbeck, M., Martinic-Kavur, M., Ladurner, R., Alexander, S., Peters, J.M. \& Ellenberg, J.

Experimental and computational framework for a dynamic protein atlas of human cell division. Nature 561, 411-415 (2018).

41. Cattoglio, C., Pustova, I., Walther, N., Ho, J.J., Hantsche-Grininger, M., Inouye, C.J., Hossain, M.J., Daily, G.M., Ellenberg, J., Darzacq, X., Tjian, R. \& Hansen, A.S. Determining cellular CTCF and cohesin abundances to constrain 3D genome models. BioRxiv (2018).

42. Owens, N., Papadopoulou, T., Festuccia, N., Tachtsidi, A., Gonzalez, I., Dubois, A., VandormaelPournin, S., Nora, E.P., Bruneau, B.G., Cohen-Tannoudji, M. \& Navarro, P. CTCF confers local nucleosome resiliency after DNA replication and during mitosis. Elife 8(2019).

43. Stadhouders, R., Vidal, E., Serra, F., Di Stefano, B., Le Dily, F., Quilez, J., Gomez, A., Collombet, S., Berenguer, C., Cuartero, Y., Hecht, J., Filion, G.J., Beato, M., Marti-Renom, M.A. \& Graf, T. Transcription factors orchestrate dynamic interplay between genome topology and gene regulation during cell reprogramming. Nat Genet 50, 238-249 (2018).

44. Whyte, W.A., Orlando, D.A., Hnisz, D., Abraham, B.J., Lin, C.Y., Kagey, M.H., Rahl, P.B., Lee, T.I. \& Young, R.A. Master transcription factors and mediator establish super-enhancers at key cell identity genes. Cell 153, 307-19 (2013).

45. Grosveld, F., van Assendelft, G.B., Greaves, D.R. \& Kollias, G. Position-independent, high-level expression of the human beta-globin gene in transgenic mice. Cell 51, 975-85 (1987).

46. Ing-Simmons, E., Seitan, V.C., Faure, A.J., Flicek, P., Carroll, T., Dekker, J., Fisher, A.G., Lenhard, B. \& Merkenschlager, M. Spatial enhancer clustering and regulation of enhancer-proximal genes by cohesin. Genome Res 25, 504-13 (2015).

47. Beagrie, R.A., Scialdone, A., Schueler, M., Kraemer, D.C., Chotalia, M., Xie, S.Q., Barbieri, M., de Santiago, I., Lavitas, L.M., Branco, M.R., Fraser, J., Dostie, J., Game, L., Dillon, N., Edwards, P.A., Nicodemi, M. \& Pombo, A. Complex multi-enhancer contacts captured by genome architecture mapping. Nature 543, 519-524 (2017).

48. Crane, E., Bian, Q., McCord, R.P., Lajoie, B.R., Wheeler, B.S., Ralston, E.J., Uzawa, S., Dekker, J. \& Meyer, B.J. Condensin-driven remodelling of $X$ chromosome topology during dosage compensation. Nature 523, 240-4 (2015). 
49. Krijger, P.H., Di Stefano, B., de Wit, E., Limone, F., van Oevelen, C., de Laat, W. \& Graf, T. Cell-ofOrigin-Specific 3D Genome Structure Acquired during Somatic Cell Reprogramming. Cell Stem Cell 18, 597-610 (2016).

50. Mumbach, M.R., Rubin, A.J., Flynn, R.A., Dai, C., Khavari, P.A., Greenleaf, W.J. \& Chang, H.Y. HiChIP: efficient and sensitive analysis of protein-directed genome architecture. Nat Methods 13, 919922 (2016).

51. Beagan, J.A. \& Phillips-Cremins, J.E. On the existence and functionality of topologically associating domains. Nat Genet 52, 8-16 (2020).

52. Giorgetti, L., Lajoie, B.R., Carter, A.C., Attia, M., Zhan, Y., Xu, J., Chen, C.J., Kaplan, N., Chang, H.Y., Heard, E. \& Dekker, J. Structural organization of the inactive $X$ chromosome in the mouse. Nature 535, 575-9 (2016).

53. Darrow, E.M., Huntley, M.H., Dudchenko, O., Stamenova, E.K., Durand, N.C., Sun, Z., Huang, S.C., Sanborn, A.L., Machol, I., Shamim, M., Seberg, A.P., Lander, E.S., Chadwick, B.P. \& Aiden, E.L. Deletion of DXZ4 on the human inactive $\mathrm{X}$ chromosome alters higher-order genome architecture. Proc Natl Acad Sci U S A 113, E4504-12 (2016).

54. Deng, X., Ma, W., Ramani, V., Hill, A., Yang, F., Ay, F., Berletch, J.B., Blau, C.A., Shendure, J., Duan, Z., Noble, W.S. \& Disteche, C.M. Bipartite structure of the inactive mouse X chromosome. Genome Biol 16, 152 (2015).

55. Kentepozidou, E., Aitken, S.J., Feig, C., Stefflova, K., Ibarra-Soria, X., Odom, D.T., Roller, M. \& Flicek, P. Clustered CTCF binding is an evolutionary mechanism to maintain topologically associating domains. BioRxiv (2019).

56. Gates, L.A., Foulds, C.E. \& O'Malley, B.W. Histone Marks in the 'Driver's Seat': Functional Roles in Steering the Transcription Cycle. Trends Biochem Sci 42, 977-989 (2017).

57. Aranda, S., Mas, G. \& Di Croce, L. Regulation of gene transcription by Polycomb proteins. Sci Adv 1, e1500737 (2015).

58. Zhou, Y., Zhou, B., Pache, L., Chang, M., Khodabakhshi, A.H., Tanaseichuk, O., Benner, C. \& Chanda, S.K. Metascape provides a biologist-oriented resource for the analysis of systems-level datasets. Nat Commun 10, 1523 (2019).

59. Boers, R., Boers, J., de Hoon, B., Kockx, C., Ozgur, Z., Molijn, A., van, I.W., Laven, J. \& Gribnau, J. Genome-wide DNA methylation profiling using the methylation-dependent restriction enzyme LpnPI. Genome Res 28, 88-99 (2018).

60. Bell, A.C. \& Felsenfeld, G. Methylation of a CTCF-dependent boundary controls imprinted expression of the lgf2 gene. Nature 405, 482-5 (2000).

61. Hark, A.T., Schoenherr, C.J., Katz, D.J., Ingram, R.S., Levorse, J.M. \& Tilghman, S.M. CTCF mediates methylation-sensitive enhancer-blocking activity at the H19/lgf2 locus. Nature 405, 486-9 (2000).

62. Wang, H., Maurano, M.T., Qu, H., Varley, K.E., Gertz, J., Pauli, F., Lee, K., Canfield, T., Weaver, M., Sandstrom, R., Thurman, R.E., Kaul, R., Myers, R.M. \& Stamatoyannopoulos, J.A. Widespread plasticity in CTCF occupancy linked to DNA methylation. Genome Res 22, 1680-8 (2012). 
63. Wiehle, L., Thorn, G.J., Raddatz, G., Clarkson, C.T., Rippe, K., Lyko, F., Breiling, A. \& Teif, V.B. DNA (de)methylation in embryonic stem cells controls CTCF-dependent chromatin boundaries. Genome Res 29, 750-761 (2019).

64. Caudron-Herger, M., Rusin, S.F., Adamo, M.E., Seiler, J., Schmid, V.K., Barreau, E., Kettenbach, A.N. \& Diederichs, S. R-DeeP: Proteome-wide and Quantitative Identification of RNA-Dependent Proteins by Density Gradient Ultracentrifugation. Mol Cell 75, 184-199 e10 (2019).

65. Hansen, A.S., Amitai, A., Cattoglio, C., Tjian, R. \& Darzacq, X. Guided nuclear exploration increases CTCF target search efficiency. Nat Chem Biol (2019).

66. Agarwal, H., Reisser, M., Wortmann, C. \& Gebhardt, J.C.M. Direct Observation of Cell-CycleDependent Interactions between CTCF and Chromatin. Biophys J 112, 2051-2055 (2017).

67. Oomen, M.E., Hansen, A.S., Liu, Y., Darzacq, X. \& Dekker, J. CTCF sites display cell cycledependent dynamics in factor binding and nucleosome positioning. Genome Res 29, 236-249 (2019).

68. Hansen, A.S., Cattoglio, C., Darzacq, X. \& Tjian, R. Recent evidence that TADs and chromatin loops are dynamic structures. Nucleus 9, 20-32 (2018).

69. Fudenberg, G., Imakaev, M., Lu, C., Goloborodko, A., Abdennur, N. \& Mirny, L.A. Formation of Chromosomal Domains by Loop Extrusion. Cell Rep 15, 2038-49 (2016).

70. Stik, G., Vidal, E., Barrero, M., Cuartero, S., Vila-Casadesus, M., Mendieta-Esteban, J., Tian, T.V., Choi, J., Berenguer, C., Abad, A., Borsari, B., leDily, F., Cramer, P., Marti-Renom, M.A., Stadhouders, R. \& Graf, T. CTCF is dispensable for immune cell transdifferentiation but facilitates an acute inflammatory response. Nature Genetics In press(2020).

71. Sabari, B.R., Dall'Agnese, A., Boija, A., Klein, I.A., Coffey, E.L., Shrinivas, K., Abraham, B.J., Hannett, N.M., Zamudio, A.V., Manteiga, J.C., Li, C.H., Guo, Y.E., Day, D.S., Schuijers, J., Vasile, E., Malik, S., Hnisz, D., Lee, T.I., Cisse, II, Roeder, R.G., Sharp, P.A., Chakraborty, A.K. \& Young, R.A. Coactivator condensation at super-enhancers links phase separation and gene control. Science 361(2018).

72. Heinz, S., Texari, L., Hayes, M.G.B., Urbanowski, M., Chang, M.W., Givarkes, N., Rialdi, A., White, K.M., Albrecht, R.A., Pache, L., Marazzi, I., Garcia-Sastre, A., Shaw, M.L. \& Benner, C. Transcription Elongation Can Affect Genome 3D Structure. Cell 174, 1522-1536 e22 (2018).

73. Busslinger, G.A., Stocsits, R.R., van der Lelij, P., Axelsson, E., Tedeschi, A., Galjart, N. \& Peters, J.M. Cohesin is positioned in mammalian genomes by transcription, CTCF and Wapl. Nature 544, 503-507 (2017).

74. Smith, Z.D. \& Meissner, A. DNA methylation: roles in mammalian development. Nat Rev Genet 14, 204-20 (2013).

75. Filippova, G.N., Qi, C.F., Ulmer, J.E., Moore, J.M., Ward, M.D., Hu, Y.J., Loukinov, D.I., Pugacheva, E.M., Klenova, E.M., Grundy, P.E., Feinberg, A.P., Cleton-Jansen, A.M., Moerland, E.W., Cornelisse, C.J., Suzuki, H., Komiya, A., Lindblom, A., Dorion-Bonnet, F., Neiman, P.E., Morse, H.C., 3rd, Collins, S.J. \& Lobanenkov, V.V. Tumor-associated zinc finger mutations in the CTCF transcription factor selectively alter tts DNA-binding specificity. Cancer Res 62, 48-52 (2002).

76. Bailey, C.G., Gupta, S., Metierre, C., Amarasekera, P.M.S., O’Young, P., Kyaw, W., Laletin, T., Francis, H., Semaan, C., Singh, K.P., Mullighan, C.G., Wolkenhauer, O., Schmitz, U. \& Rasko, J.E. 
Somatic mutations in CTCF zinc fingers produce cellular phenotypes explained by structure- function relationships. BioRxiv (2021).

77. Chen, X., Xu, H., Yuan, P., Fang, F., Huss, M., Vega, V.B., Wong, E., Orlov, Y.L., Zhang, W., Jiang, J., Loh, Y.H., Yeo, H.C., Yeo, Z.X., Narang, V., Govindarajan, K.R., Leong, B., Shahab, A., Ruan, Y., Bourque, G., Sung, W.K., Clarke, N.D., Wei, C.L. \& Ng, H.H. Integration of external signaling pathways with the core transcriptional network in embryonic stem cells. Cell 133, 1106-17 (2008). 


\section{Figure Legends}

Figure 1. Generation of CTCF ZF deletion ES cell lines and genomic binding profile of mutant CTCF proteins.

a) Amino acids deleted in CTCF zinc finger (ZF) deletions 1, and 8-11. Cysteines (C) and histidines $(\mathrm{H})$ important for zinc coordination in ZFs 1 and 8-11 are underlined. The red coloured amino acids (K, D -> E) in the del1 (d1) mutant indicate an unintended extra amino acid deletion $(\mathrm{K})$ at the $\mathrm{N}$-terminus of the intended deletion, and the unintended presence of a mutated amino acid (E) instead of the intended deletion (D), located at the C-terminus of the deleted amino acid stretch (see also Extended data Fig. 1b).

b) Western blot analysis of wild type (WT) and rescued ES cell lines expressing GFP-CTCF (GC), or GFP-CTCF-del8, -del9, -del10, -del11, or -del1. Blots were incubated with anti-CTCF (upper panel), anti-GFP (middle panel), or anti-UBF (lower panel) antibodies.

c) ChIP-Seq analysis of wild type and mutant CTCF binding in ES cells expressing the indicated CTCF proteins (left). Binding scale is shown to the right. Previously published CTCF ChIP-Seq peaks and binding sites (rectangles) in ES cells are shown in the 'ES' track ${ }^{77}$.

d) K-means clustering of CTCF binding sites showing at least a four-fold change in binding for at least one mutant protein, revealing 6 subgroups. Scale represents log2-fold change.

e) Motif analysis of the GFP-CTCF consensus binding site (top row), and of sites with more than 8 fold reduced binding in the indicated ZF mutants. Core (C), upstream (U), and downstream (D) motifs are indicated, as are the ZFs that may bind these regions.

Figure 2. Interphase localization and dynamic behaviour of CTCF proteins.

a) Intracellular distribution of fluorescent CTCF (green) in ES cells expressing GFP-CTCF or del8. DNA was visualized using SiR-Hoechst (red). Scale bar : 8 micron.

b) Fluorescence recovery after photobleaching (FRAP) experiments in ES cells and Monte Carlo simulations of FRAP data. Left panels show a representative FRAP experiment, with still images of a colony of GFP-CTCF-expressing ES cells before (Pre-bleach), immediately after (Bleach) and at the end of the FRAP experiment (Post-bleach). White circles indicate bleached regions of interest. Scale bar: 10 micron. The right upper panel shows the results of FRAP experiments in ES cell lines expressing GFP-CTCF (GC, black) or the indicated mutants (del1: red, del8: green, del9: blue, del10: orange, del11: yellow). Average values of 12-23 FRAP experiments are shown. For clarity we did not indicate SEMs. The right lower panel shows Monte Carlo simulations of FRAP data (GC, experimentally determined curve 
in light blue; simulated curve in yellow) and for del8 (experimentally determined curve in blue; simulated curve in green).

c) Biophysical properties of GFP-CTCF and GFP-CTCF-del8 as determined by Monte Carlo simulations. Note that the $K_{o n}$ is the effective on-rate; i. f.: immobile fraction, r.t. : residence time (or dwell time) of CTCF on DNA.

d) Fluorescence correlation spectroscopy (FCS) experiments in ES cells expressing GFPtagged CTCF. Upper left panels show still images of ES cells expressing GFP-CTCF (GC) or del8 (d8), cross hair indicates the point of FCS measurement. Scale bar: 3 micron. Fitting of the autocorrelation curves revealed two populations of diffuse nuclear GFP-CTCF and one for del8. $\mathrm{D}$ : diffusion coefficient, $\mathrm{F}$ : fraction of total population. The box plot displays concentrations of soluble (= non DNA bound) GFP-CTCF and del8. Boxplots are shown with median, 25/75\% quartiles, and SD (GC: $n=75$ individual curves from 20 cells, $d 8: n=30$ individual curves from 8 cells).

Figure 3. Mitotic localization and dynamic behaviour of CTCF proteins.

a) Still images of time lapse movie of GFP-CTCF-expressing ES cell colony, in which two cells undergo mitosis. NEB = nuclear envelope breakdown, $\mathrm{M}=$ metaphase, $\mathrm{A}=$ anaphase , $\mathrm{C}=$ cytokinesis, $\mathrm{d} 1, \mathrm{~d} 2=$ daughter cell 1 , and 2 . Scale bar : 10 micron. See Extended data Movie 1 for the complete time lapse experiment. Scale bar : 10 micron.

b) Fluorescence intensity (F.I.) in arbitray units (A.U.) of chromosomal regions of interest (ROls) over time in cell 1 , and cell 2.

c) Fluorescence recovery after photobleaching (FRAP) experiments on ES cells expressing GFP-CTCF. Still images are of a GFP-CTCF-expressing ES cell in metaphase analyzed by FRAP. A metaphase chromosome, with bound GFP-CTCF, is shown before (Pre-bleach), immediately after (Bleach) and at the end of the FRAP experiment. The white dotted circle indicates the bleached ROI. Scale bar : 3 micron. The line graph shows the results of FRAP experiments in metaphase (black, $n=13$ ) and interphase (red, $n=7$ ) ES cell lines expressing GFP-CTCF. Average values +/- SEM are shown.

d, e) Binding of GFP-CTCF, del9 and del8 to mitotic chromosomes. Panel (d) shows still images of cells expressing the indicated proteins (GFP-CTCF : GC) in prophase (upper panels) or metaphase (lower panels). Scale bar : 4 micron. Panel (e) displays the ratio of the fluorescence intensity $(\mathrm{FI})$ on metaphase DNA versus the cytoplasm, as an indicator of DNA binding. T-test reveals a statistically significant difference in FI ratio between GFP-CTCF and del8 ${ }^{* *}: \mathrm{P}<0.005$, TTEST). 
Figure 4. $A / B$ compartmentalization and long-range superenhancer clustering in ES cells expressing wildtype or del8 CTCF

a) Distance decay curves of chromosome-wide interactions for wildtype (GC) and del8 (d8) expressing ES cells.

b, c) Representative genomic region showing unperturbed A/B compartmentalization in del8expressing cells as visualized by the checkboard pattern in Hi-C maps (panel b, 100kb resolution) and the first principal component (PC1) of a PCA on the Hi-C correlation matrix (panel c, 100kb resolution).

d) Absolute PC1 values of genome-wide A and B compartment regions (100kb bins) for wildtype (GC) and del8 (d8) expressing cells.

e) Genomic regions (100kb bin size) that switch compartment ( $6 \%$ of the genome) due to the del8 mutation. Bar indicates distribution of A-to-B and B-to-A switches.

f) Cartoon illustrating long-range interactions between several superenhancers (SE, A-D) located in different topological associating domains (TADs).

g) 2D (upper left inset) and 3D meta-plot quantifying inter-TAD interaction frequencies between superenhancers in wildtype or del8-expressing cells.

h) Percentages of genes transcriptionally deregulated (up or downregulated, absolute log2fold change $>0.5, F D R<0.05$ ) in del8 versus wildtype CTCF-expressing cells for indicated gene sets. SE gene sets were obtained from two independent studies ${ }^{43,44}$.

i) Expression levels of $245 \mathrm{SE}$ genes in ES cells expressing GFP-CTCF (GC) or del8 (d8). Data are based on RNA-Seq from two independently generated GC lines (analyzed in triplicate) and three del8 lines (analyzed in duplicate or triplicates).

j) Expression ( $\log 2$ RPKM) of the four indicated core pluripotency genes ${ }^{43}$ in ES cells expressing GFP-CTCF (GC) or del8 (d8). ${ }^{*} \mathrm{P}<0.05$, ${ }^{* * * *} \mathrm{P}<0.0001$ (Wald test corrected for multiple testing).

Figure 5. ZF8 deletion destabilizes TAD organization in ES cells.

a) Hi-C interaction map (20kb resolution) of a selected TAD border (indicated by dashed lines) weakened in del8-expressing (d8) ES cells compared to cells expressing GFP-CTCF (GC). Line graph below shows insulation scores across the region, with lower scores indicating stronger insulation.

b, c) Meta-plot of all TAD borders (panel b) and average contact enrichment for interactions inside/outside TADs (panel c) in GC- and d8- expressing ES cells. 
d) Genome-wide comparison of TAD border insulation scores in GC- and d8-expressing cells.

e) Average insulation scores (left) and CTCF peaks per border (right) in GC-expressing ES cells for TAD borders that are minimally affected ('stable'), lose ('loss') or gain ('gain') insulation score in del8-expressing cells. ${ }^{* *} \mathrm{P}<0.01,{ }^{* \star * *} \mathrm{P}<0.0001$ (Mann Whitney $\mathrm{U}$ test)

f) Log2-fold change in CTCF ChIP-Seq signal coverage in del8-expressing ES cells (as compared to GC cells) at CTCF sites in the three TAD border categories. ${ }^{*} \mathrm{P}<0.01$, ${ }^{* * * *} \mathrm{P}<0.0001$ (Mann Whitney $U$ test)

g) Meta-plot of Hi-C interaction signal in GC- and d8-expressing ES cells at loops ( $n=267$ ) called in GC-expressing cells..

h) Meta-plot of CTCF ChIP-Seq signal for CTCF binding sites in 'stable' and 'loss' category TAD borders.

i) Violin plot of gene expression output (sum of all RPKM values from genes within a border) by the three TAD border categories in GC-expressing ES cells. ${ }^{* * *} \mathrm{P}<0.0001$ (Mann Whitney U test)

j) Percentages of genes transcriptionally deregulated (up or downregulated, absolute log2fold change $>0.5, F D R<0.05$ ) in del8- versus GC-expressing ES cells for indicated genes at 'stable' and 'loss' category TAD borders. ${ }^{*} P<0.05$, Fisher's exact test.

k) Meta-plots of ChIP-Seq data for selected histone modifications associated with transcription elongation (H3K79Me2, $\mathrm{H} 3 \mathrm{~K} 36 \mathrm{Me} 3)$ and transcriptional repression (H3K27Me3) at CTCF binding sites within 'stable' and 'loss' category TAD borders.

Figure 6. CTCF ZF8 deletion results in transcriptional defects.

a) Differentially expressed genes (absolute log2-fold change $>0.5$, FDR $<0.05$ ) in ES cells expressing GFP-CTCF (GC) or del8 (d8). Data are based on RNA-Seq from two independently generated GC lines (analyzed in triplicate) and three del8 lines (analyzed in duplicate or triplicates).

b) Percentage of genes bound by CTCF at their promoter in wildtype ES cells shown for all genes (gray bar), or the upregulated genes (orange bar) and downregulated genes (blue bar) of panel a. ${ }^{* * *} \mathrm{P}<0.0001$; Fisher's exact test

c) Boxplot of absolute gene expression levels in ES cells expressing GFP-CTCF (GC) for the up or downregulated genes of panel $\mathbf{a} .{ }^{* * * *} \mathrm{P}<0.0001$; Mann Whitney $U$ test

d) Meta-plots of CTCF ChIP-Seq signal in GC- and del8-expressing cells for CTCF binding sites at promoters or non-promoter regions. 
e) Meta-plots of ATAC-Seq (chromatin accessibility), MNase-Seq (nucleosome positioning) and indicated ChIP-Seq data in wildtype ES cells at CTCF sites within promoters or outside promoter ('non-promoter') regions.

Figure 7. CTCF ZF8 deletion results in aberrant global DNA methylation.

a) Expression levels of Dnmt3a in GC and d8-expressing ES cells as determined by RNASeq. ${ }^{* * * *} P<0.0001$ (Wald test corrected for multiple testing)

b) Western blot analysis of cell lysates from GC or d8-expressing ES cells. Western blots were probed with antibodies against Dnmt3a or tubulin. "I" and "s" depict long and short Dnmt3a isoforms, respectively.

c) MeD-Seq analysis of DNA methylation in ES cells expressing GFP-CTCF (GC) or del8 (d8). Heatmap shows Z-scored DNA methylation levels at differentially methylated regions (DMRs) with a length of at least 100 nucleotides, containing at least $10 \mathrm{Lpnpl}$ sites and with at least a 3-fold difference in methylation. Two or three independently generated GC and del8 clones were analyzed, each clone was examined in duplicate.

d) Venn diagrams depicting the overlap between hyper-methylated DMRs and CTCF peaks in TAD borders that are lost or weakened in del8 expressing cells (top), or the overlap between hyper-methylated DMRs and CTCF peaks that are lost or reduced $(>20 \%$ ChIP-Seq signal decrease) in del8 expressing cells (bottom).

e) CTCF ChIP-Seq signals at the Dnmt3a locus in ES cells expressing GFP-CTCF (GC) or del8 (d8). H3K27Ac ChIP-Seq from wildtype ES cells is shown to highlight gene regulatory activity. Yellow shading indicates CTCF peaks that are lost in del8 expressing cells.

f) Hi-C interaction matrix (40kb resolution) showing normalized interaction frequencies around the Dnmt3a locus. TAD border calls in GC and del8-expressing ES cells are indicated by arrows and yellow dashed lines.

g) Virtual $4 \mathrm{C}$ analysis extracted from in-situ $\mathrm{Hi}-\mathrm{C}$ data showing normalized interaction counts with the promoter of the Dnmt3a short isoform in ES cells expressing GFP-CTCF (GC) and del8 (d8). H3K27Ac signals are shown on top to indicate active gene regulatory elements; arrowheads denote location of CTCF peaks that are lost upon ZF8 deletion. Yellow shading indicates $\mathrm{H} 3 \mathrm{~K} 27 \mathrm{Ac}+$ regions with increased promoter interaction frequencies in del8expressing ES cells.

h) Model for CTCF-chromatin interactions. The left-hand panels depict CTCF ZF binding versatility depending on whether only the core motif $(C)$ is present (upper panel), or whether the binding site is extended with $U$ and/or $D$ motifs (lower panel). When only the $C$ motif is 
present, ZF8 functions as an aspecific clamp through minor groove binding ${ }^{14}$. Binding to the UC or UCD motifs involves ZFs 9-11, or ZFs 1-3 and 9-11, respectively, and in these cases ZF8 acts as a linker. CTCF residence time is predicted to increase upon extension of the motif. CTCF binding to the different motifs may involve conformational changes in the protein, which could affect Cohesin blocking potential during loop extrusion. The right-hand panel depicts three levels (1 and 2: local, 3: global) at which CTCF binding efficiency is determined; 1: sequence context, 2: chromatin environment (e.g. accessibility), 3: nuclear CTCF concentration. 
a
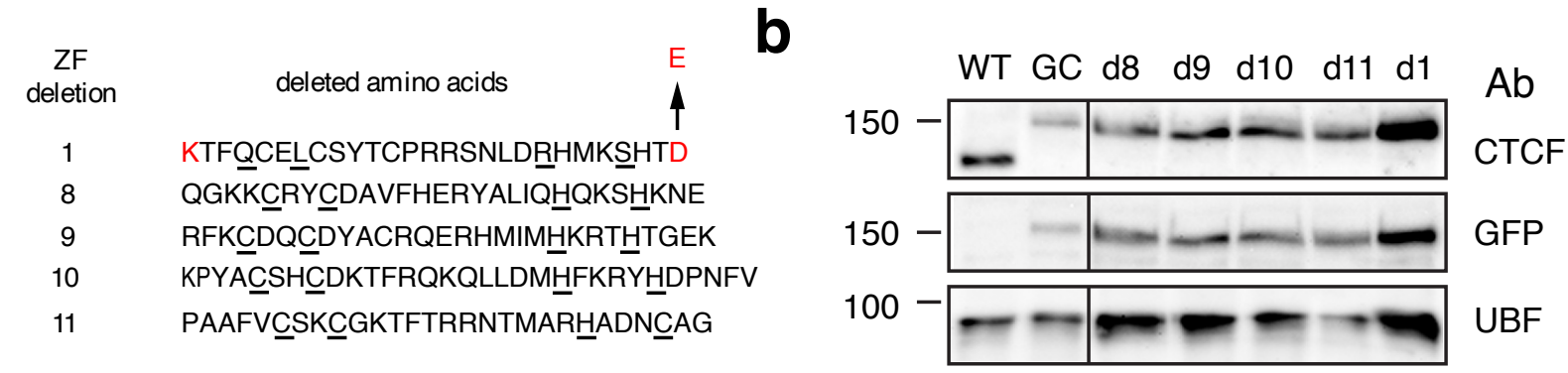

C

$153 \mathrm{~kb}$

read counts

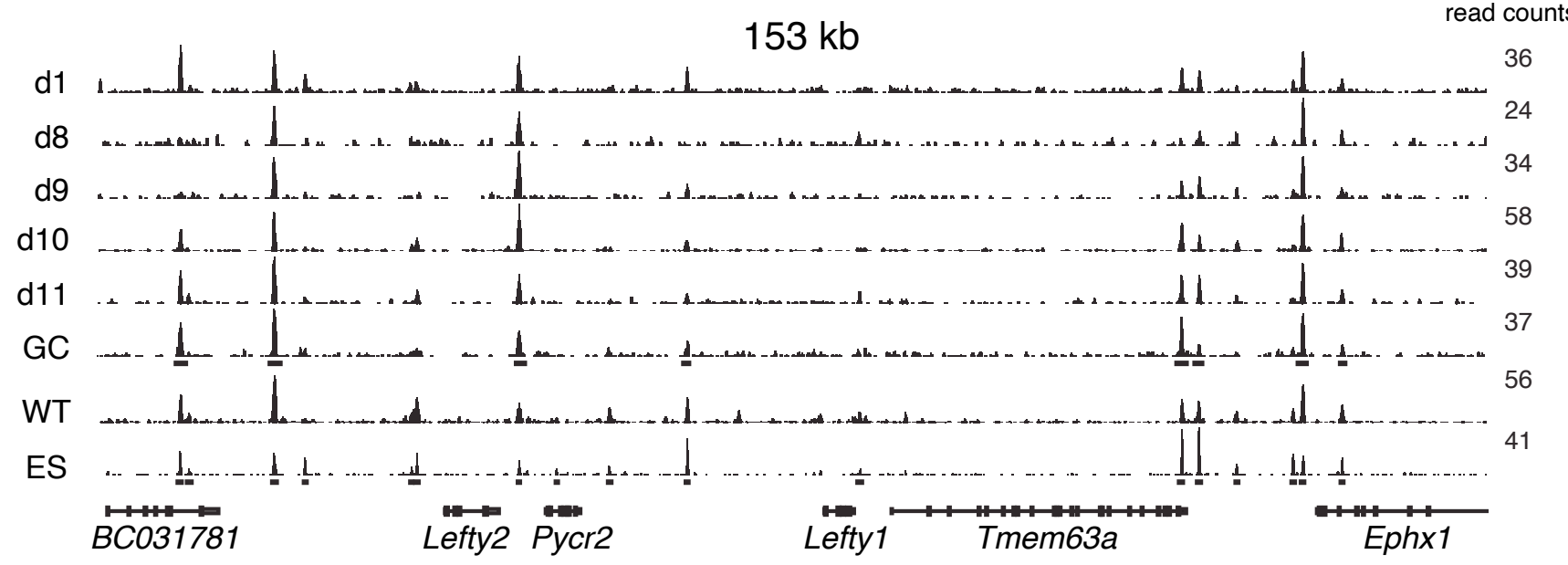

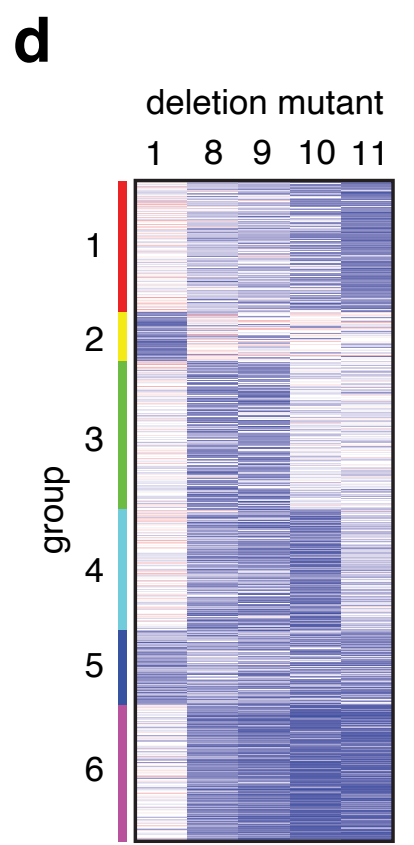

Log2

(ZF mutant vs. GFP-CTCF) e

2

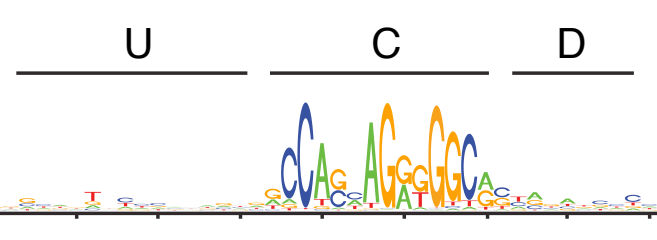

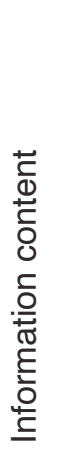

2

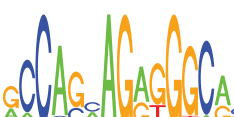

del 1

GFP-CTCF

del 8

del 9

del 10

del 11

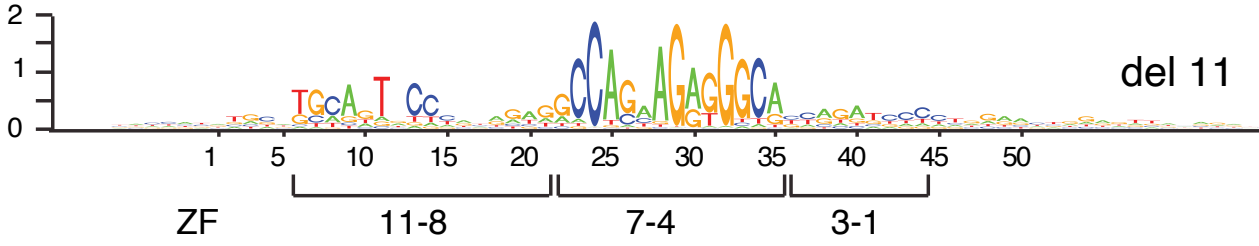

Figure 1 - Soochit 
a

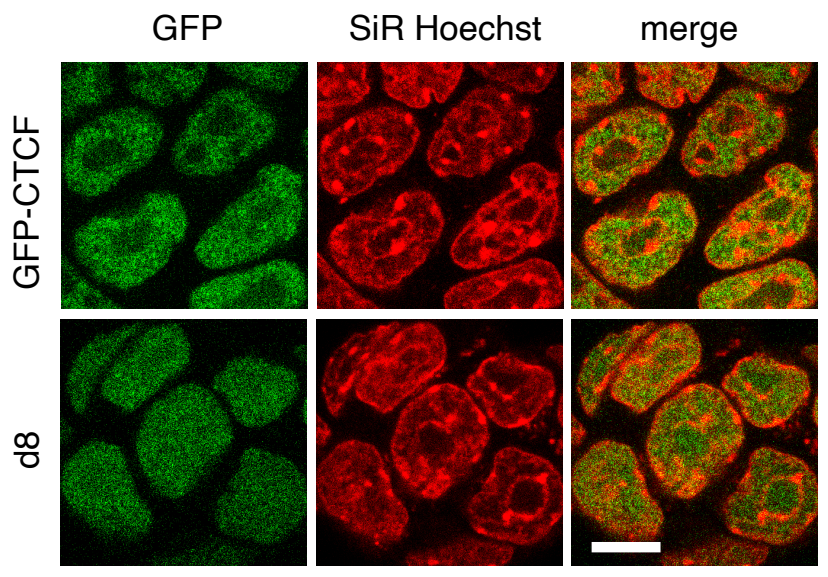

b GFP-CTCF
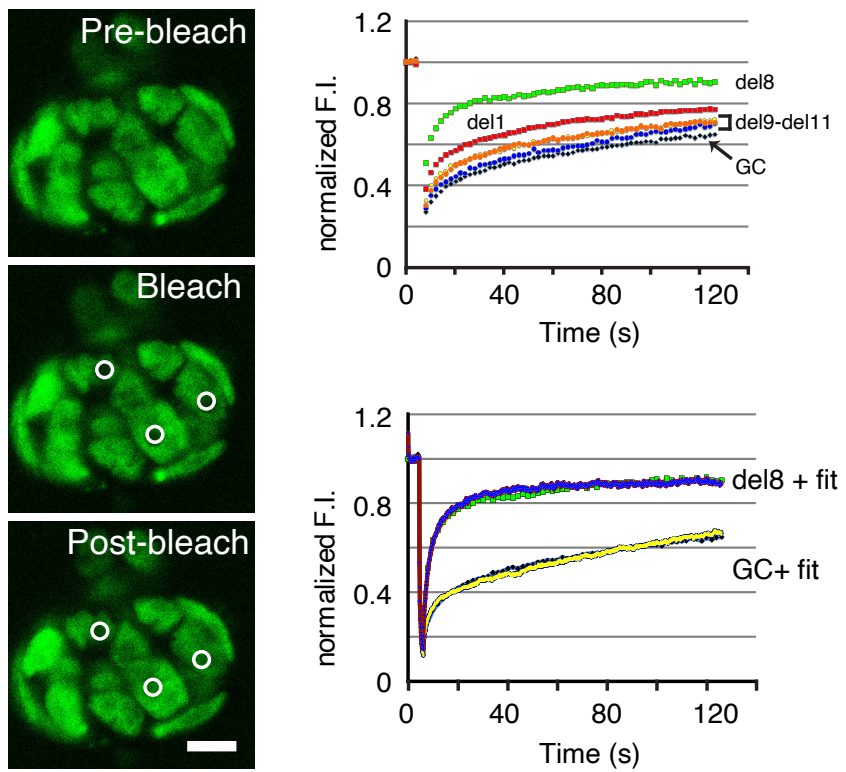

C

\begin{tabular}{|c|c|c|c|c|}
\cline { 2 - 5 } \multicolumn{1}{c|}{} & $\mathrm{k}_{\text {on }}\left(\mathrm{s}^{-1}\right)$ & $\mathrm{k}_{\text {off }}\left(\mathrm{s}^{-1}\right)$ & i.f. & r.t. $(\mathrm{s})$ \\
\hline $\mathrm{GC}$ & 0.014 & 0.0071 & 0.66 & 140 \\
del8 & 0.012 & 0.056 & 0.18 & 17.8 \\
\hline
\end{tabular}
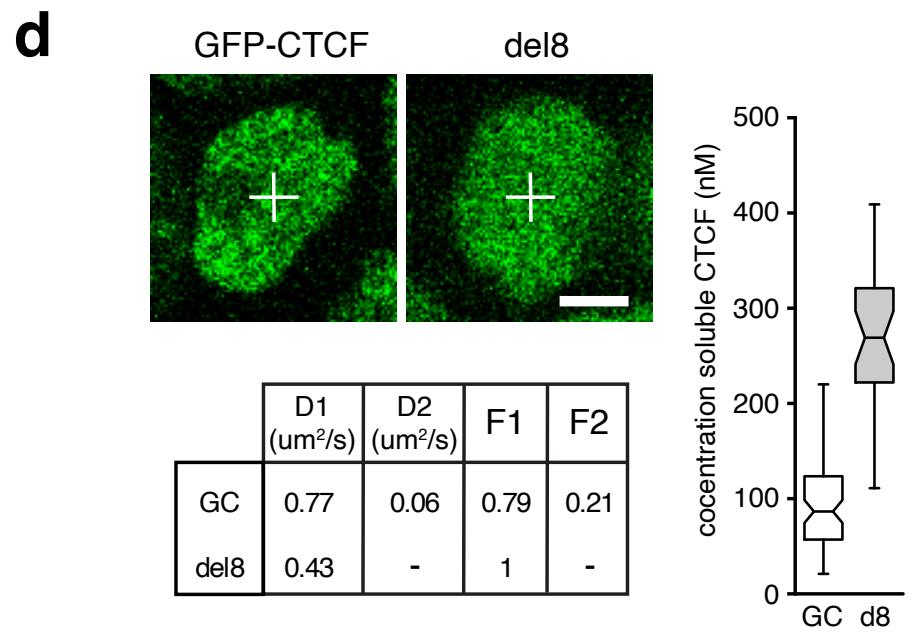
a
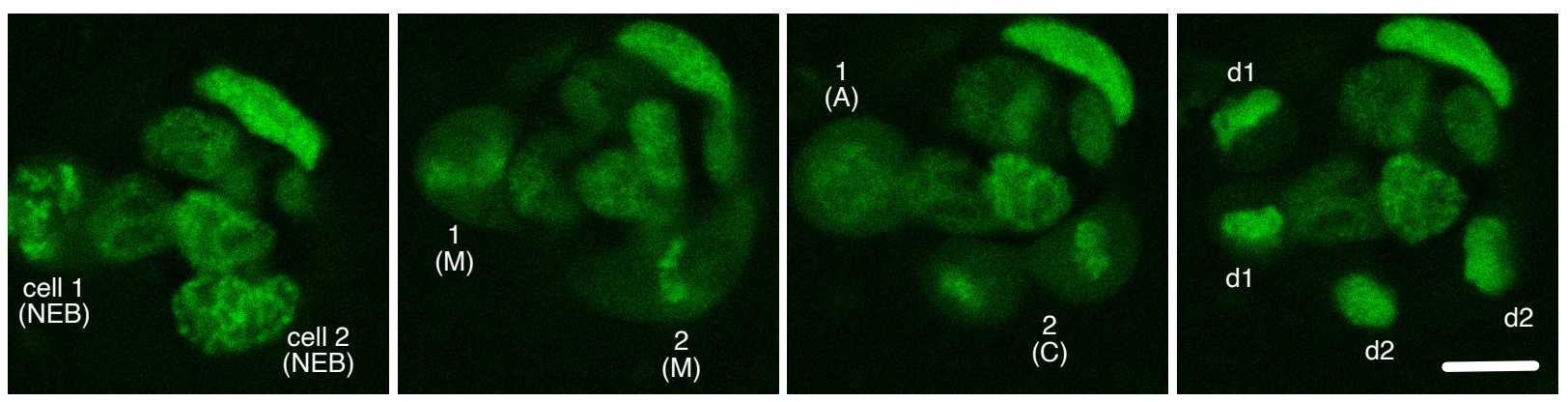

b

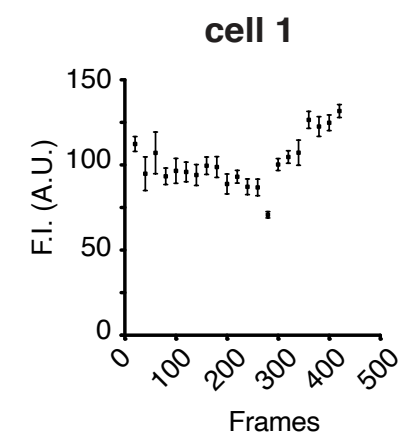

d

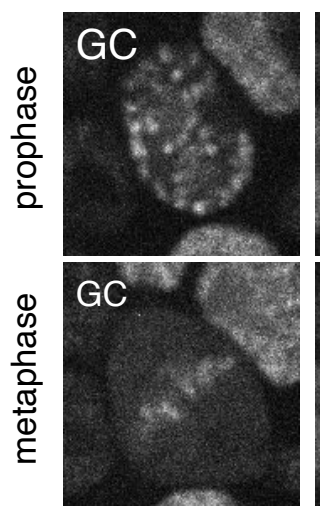

C
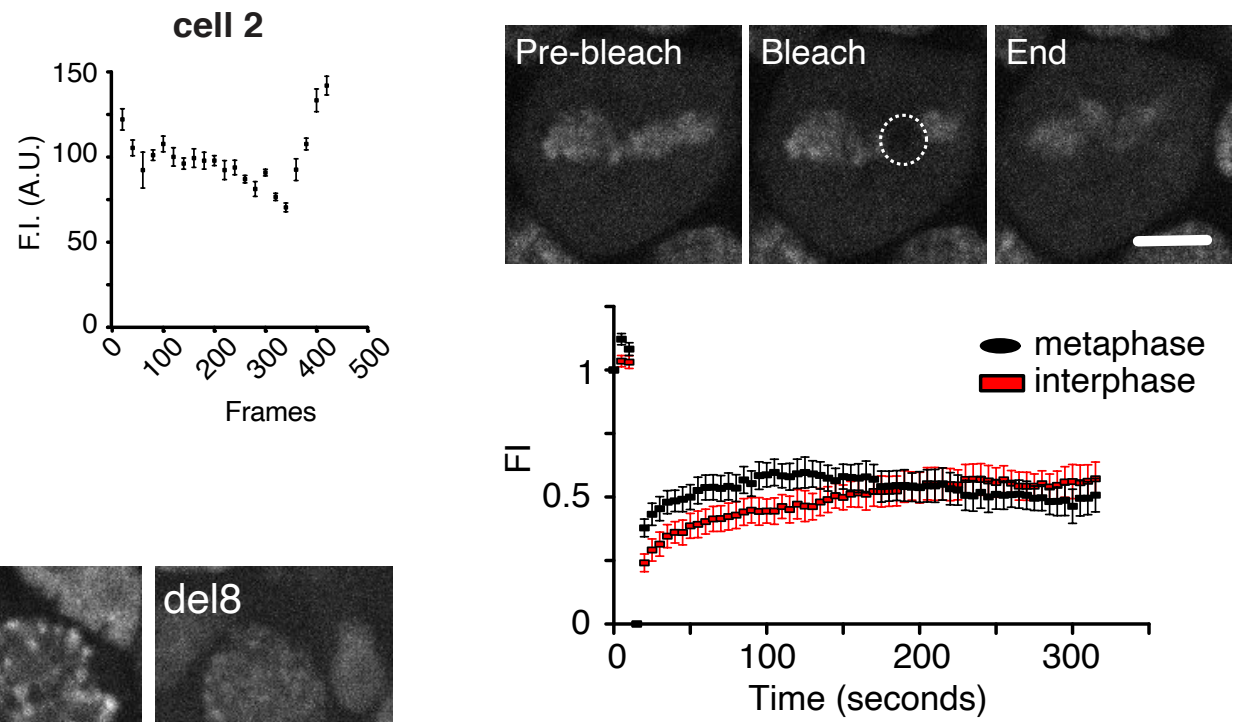

e
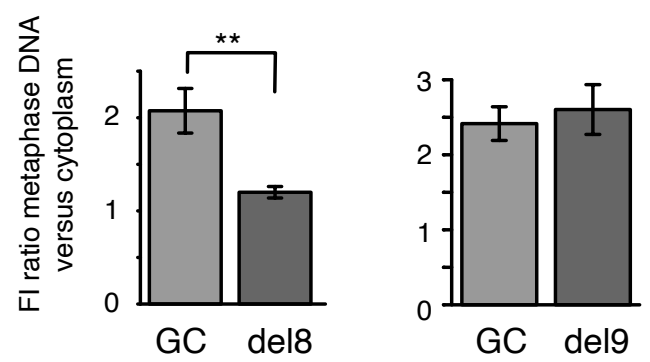
a

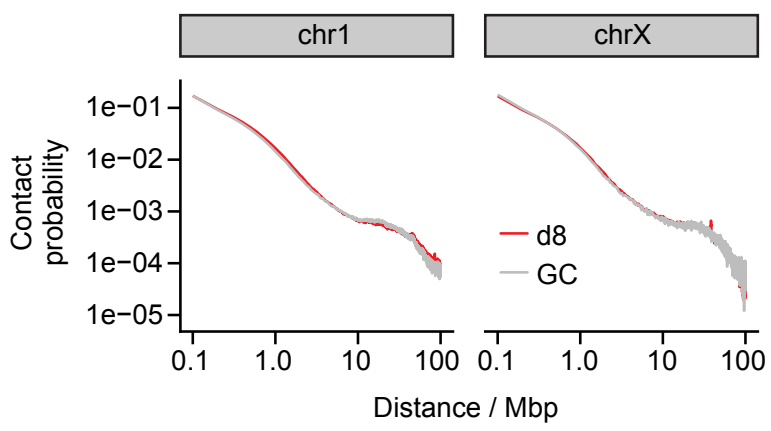

b

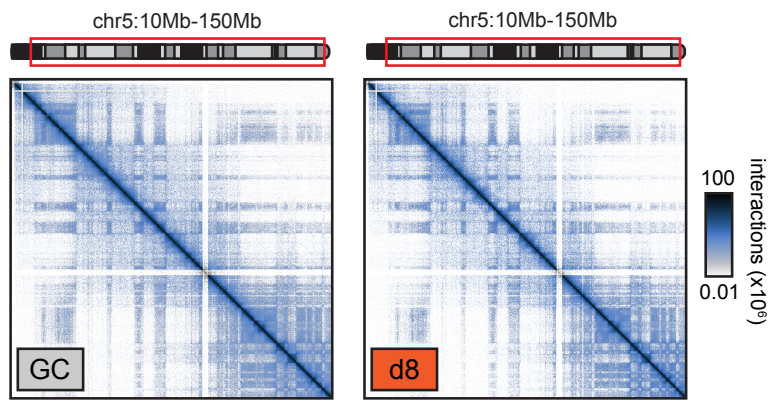

C

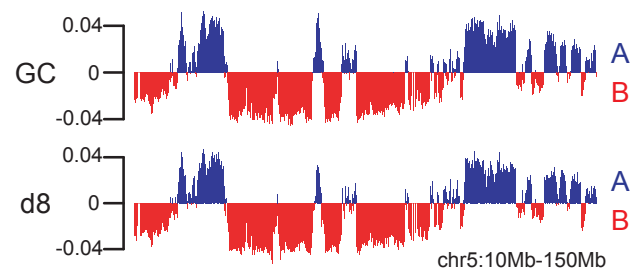

f

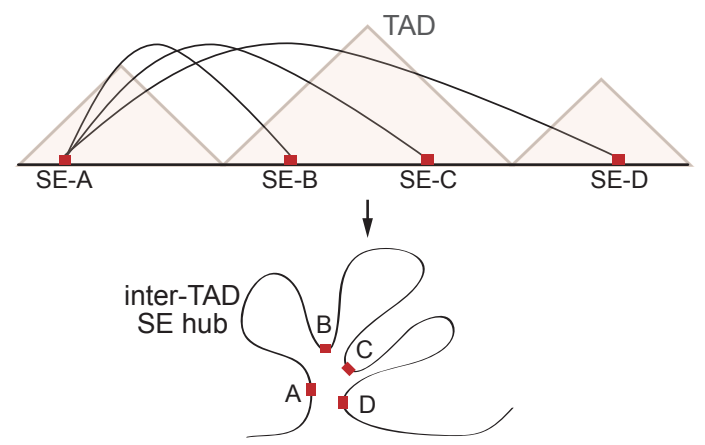

h

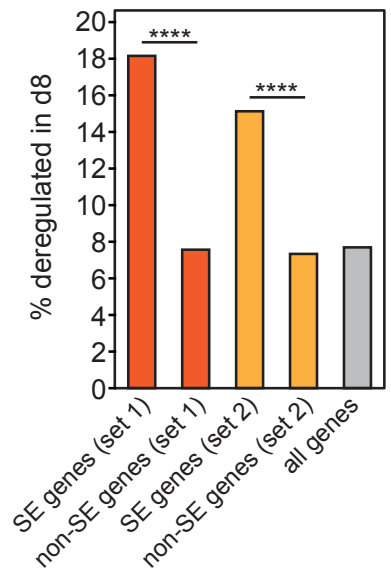

d

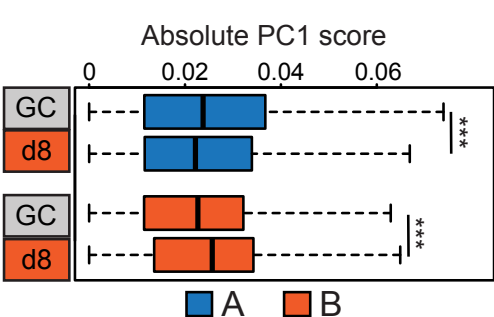

e

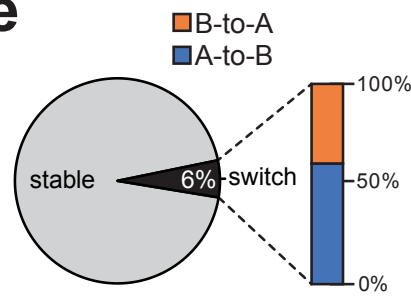

g
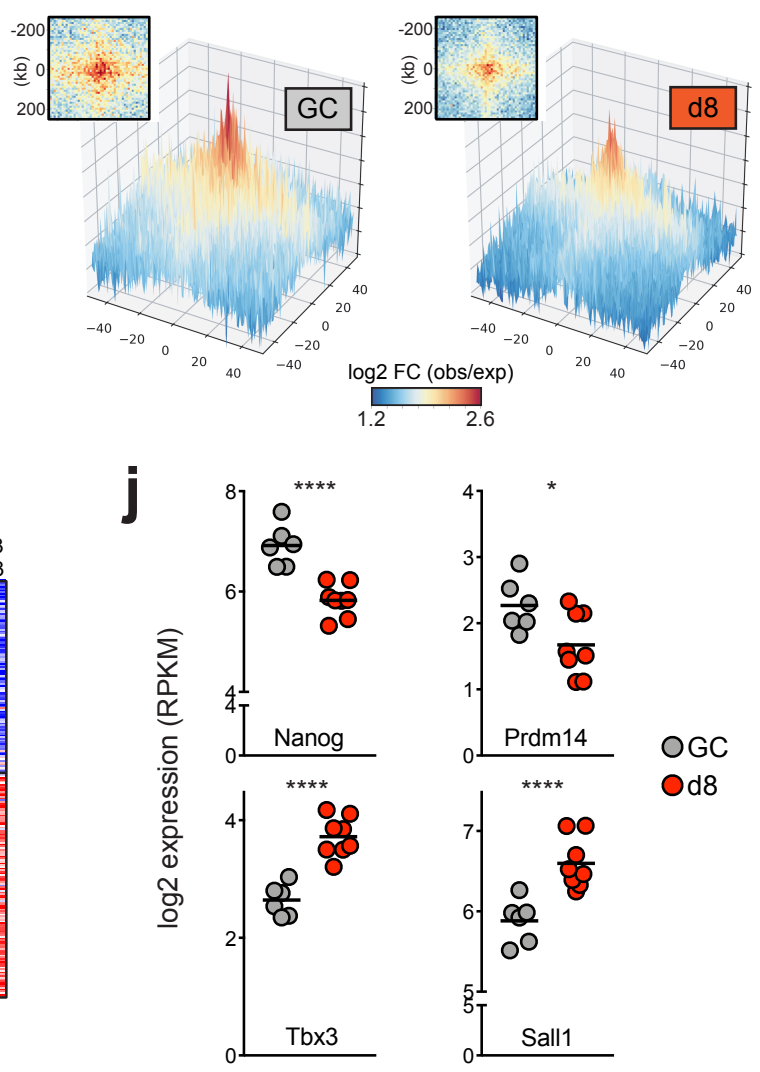
a

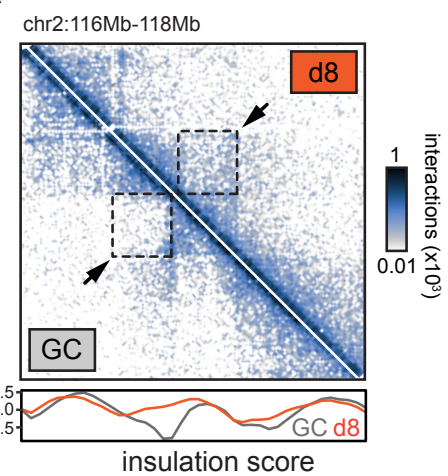

e

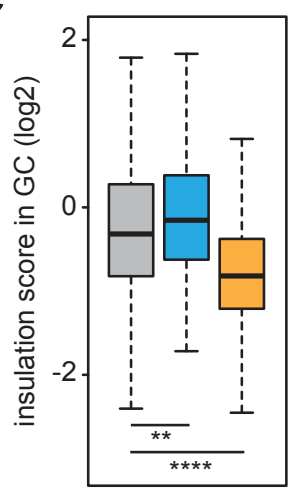

h

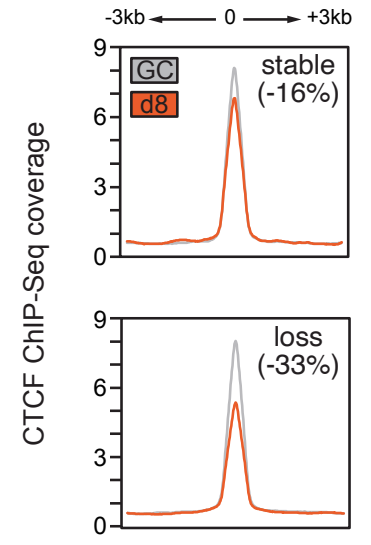

b

d8

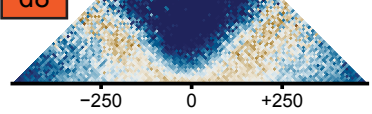

Position (relative to TAD border) / Kbp

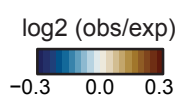

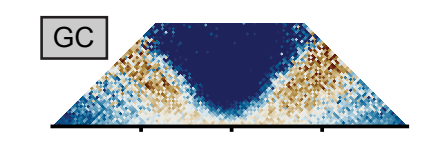

C

Contact enrichment inside/outside TAD

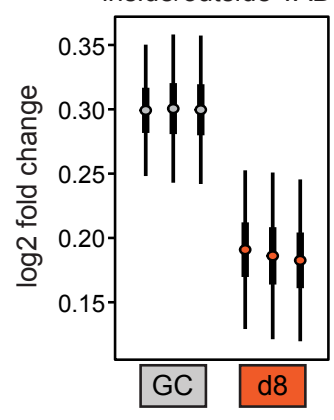

f

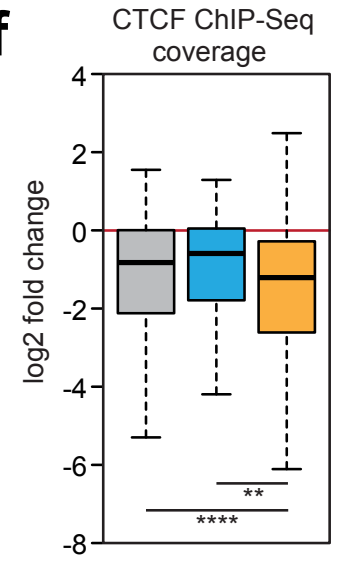

i

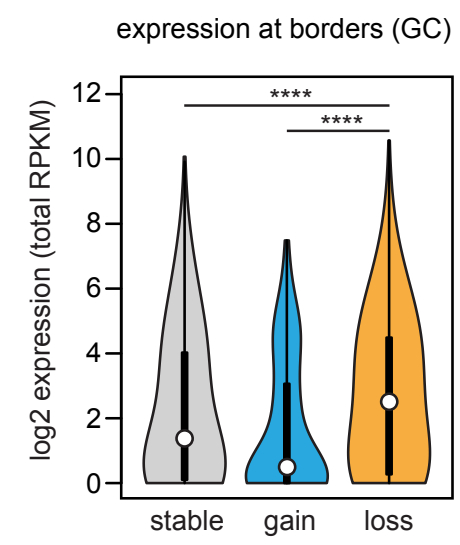

d

TAD border insulation scores

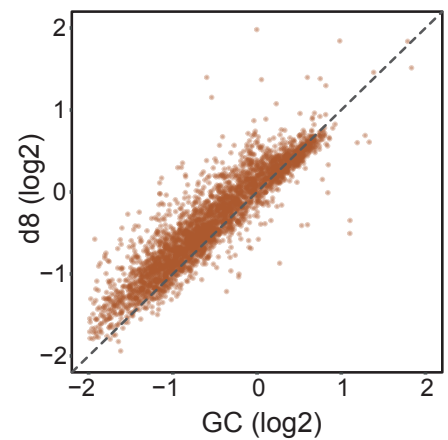

g

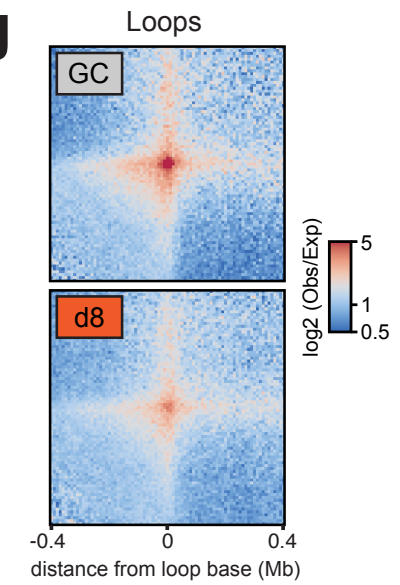

k chromatin landscape at border CTCF binding sites
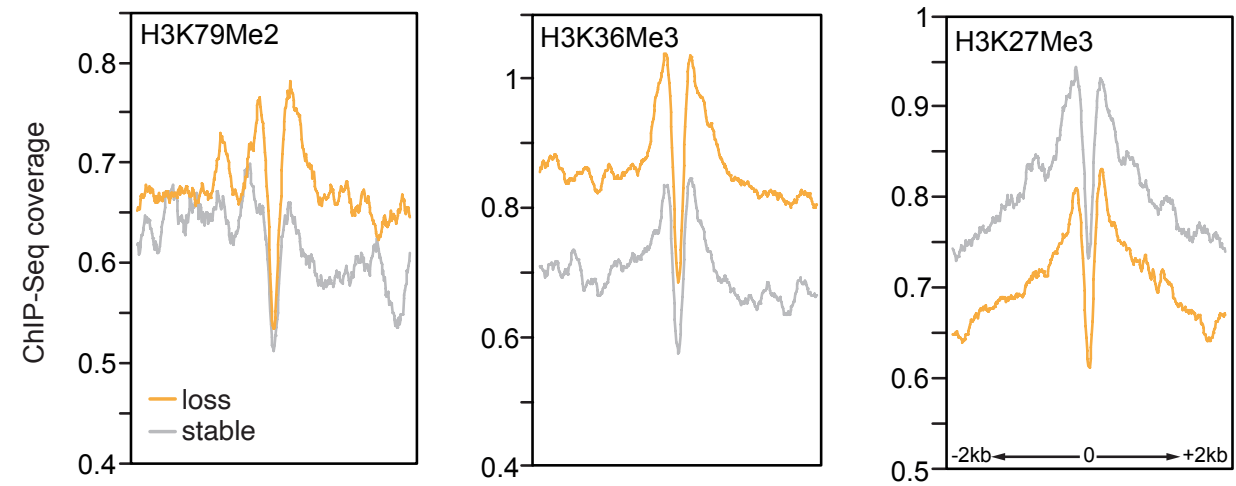

Figure 5 - Soochit 
a

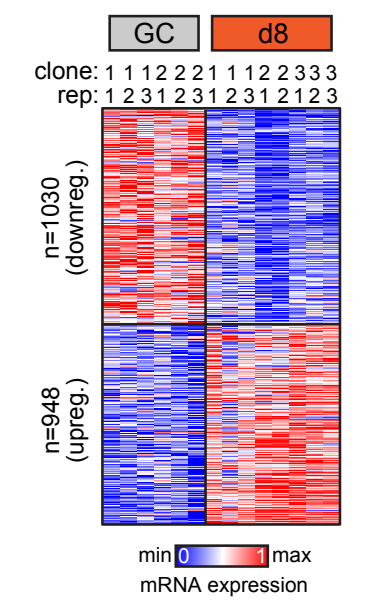

d

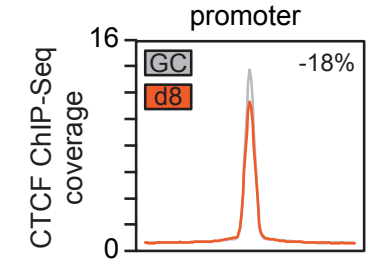

e
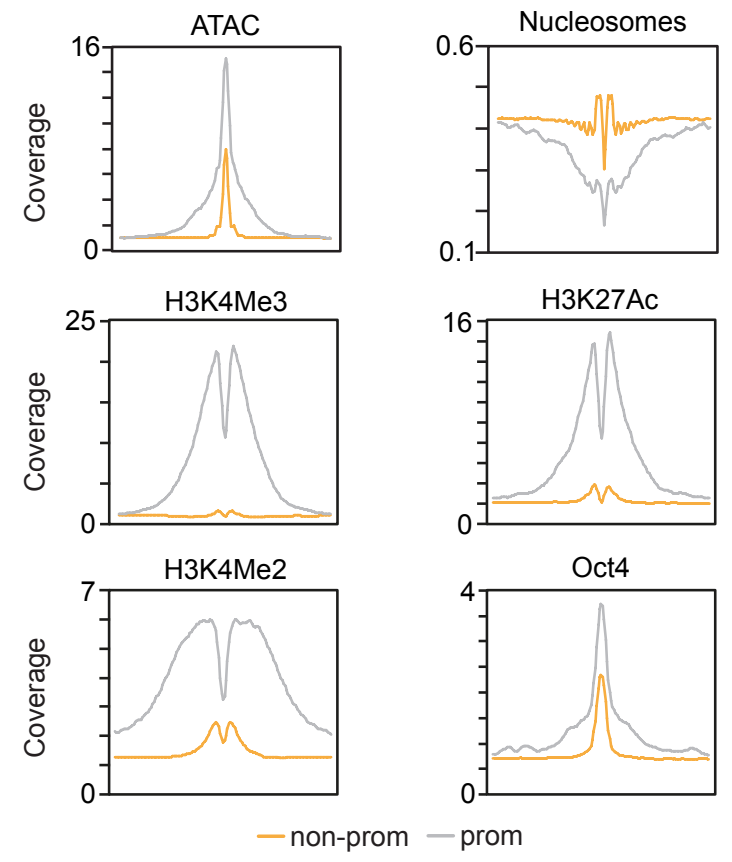

b

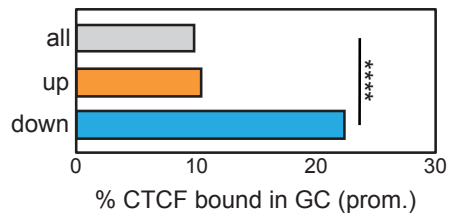

C

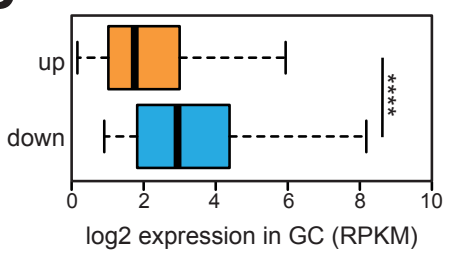

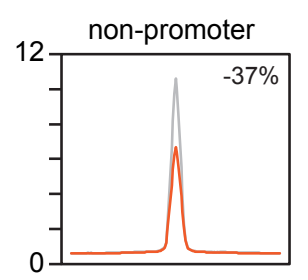
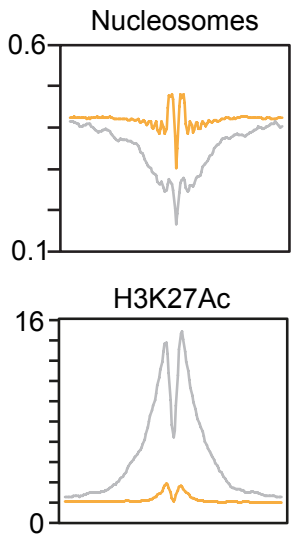

Figure 6 - Soochit 
a

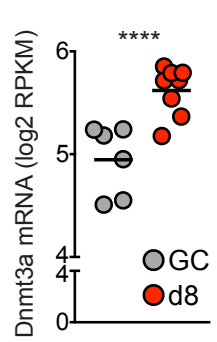

b

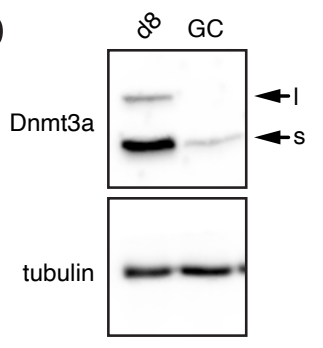

e

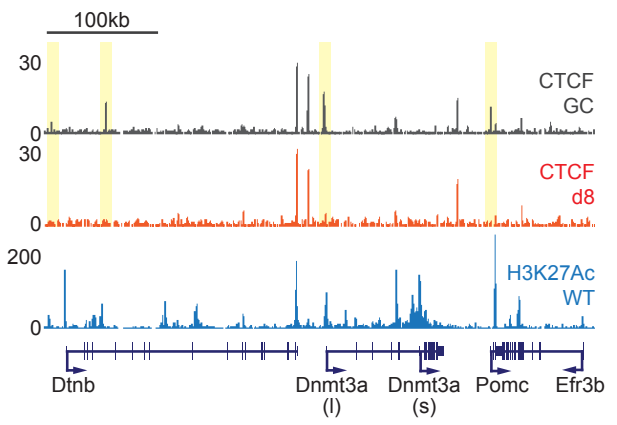

f

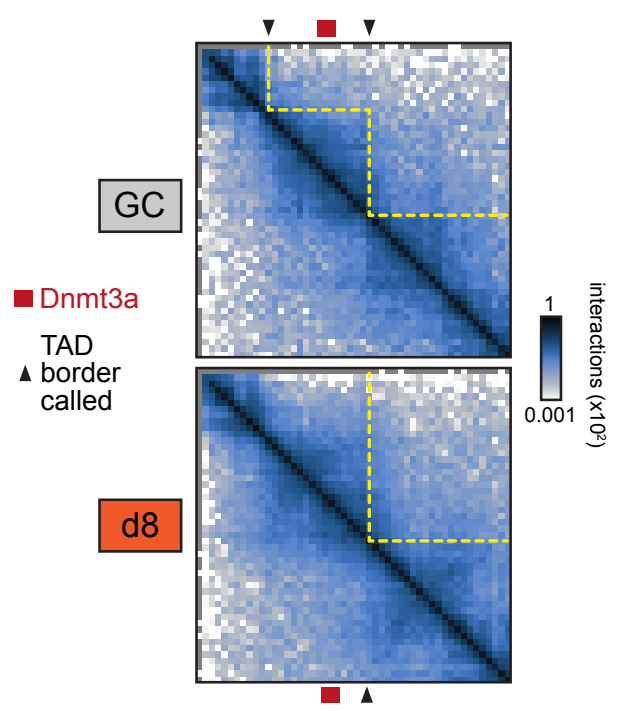

d
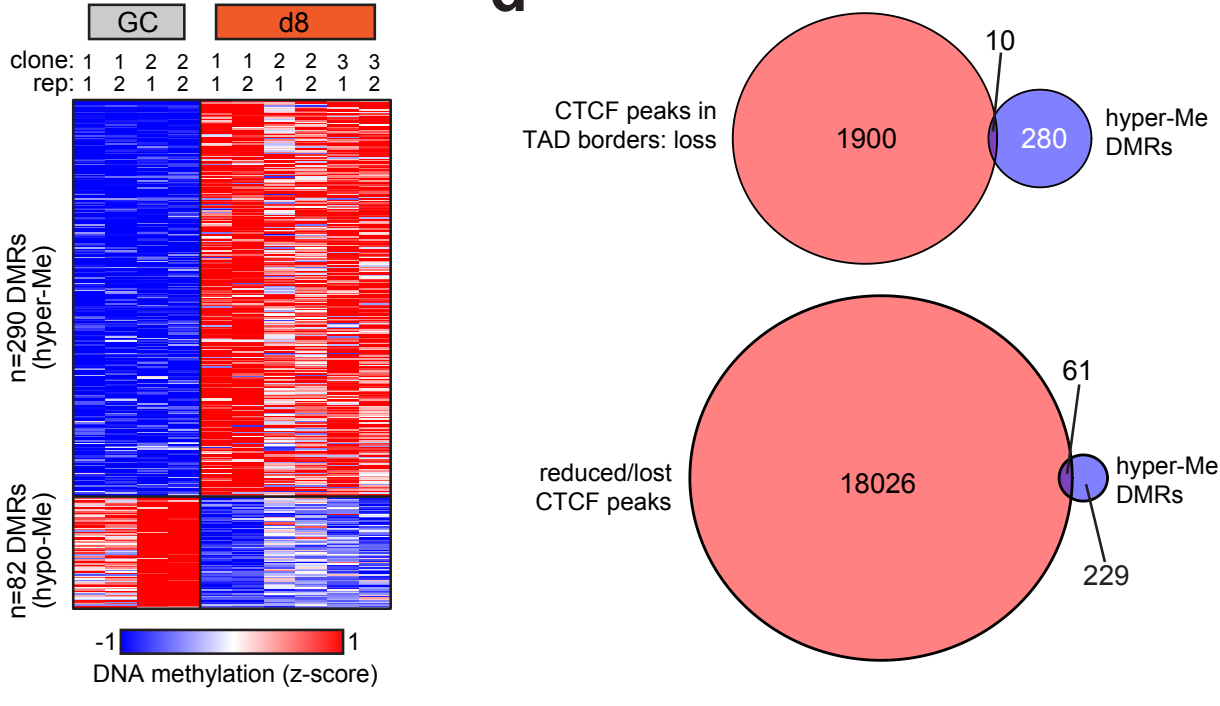

g
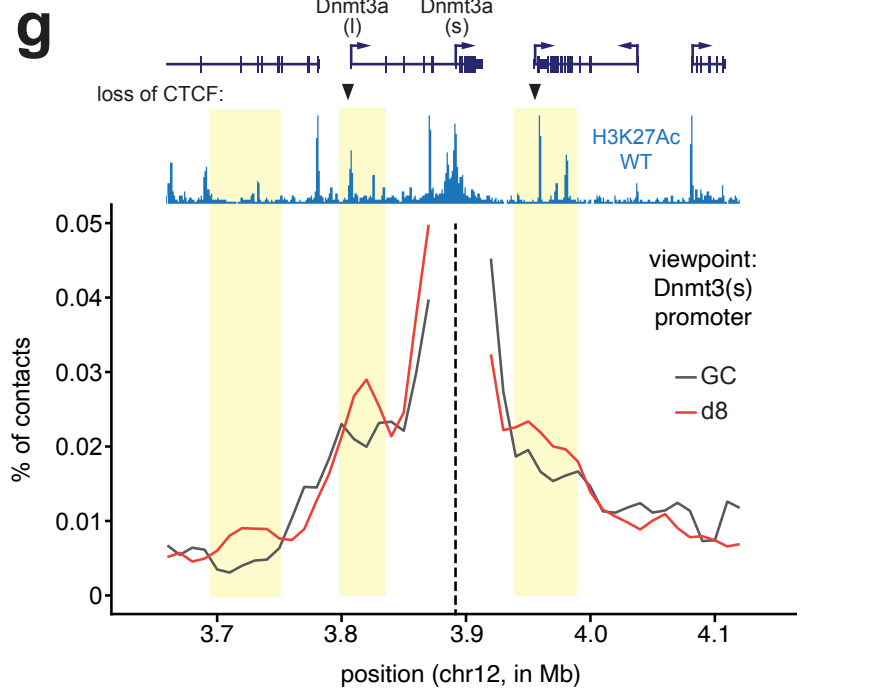

h

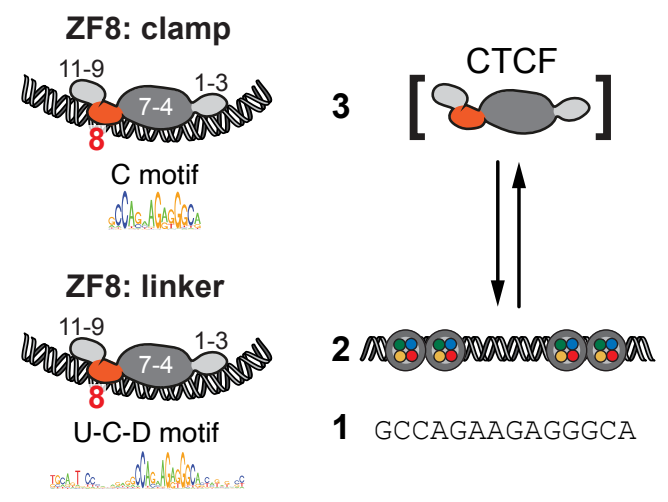




\section{Extended data}

Extended data Fig. 1. Characterization of ES cell lines expressing GFP-CTCF or mutant CTCF proteins.

a) CTCF zinc finger position and sequence. ZFs are based on ${ }^{1}$. Cysteines (C) and histidines

(H) important for zinc coordination are boxed.

b) Designed amino acid deletions in the various CTCF ZF mutant proteins.

c) Results of the ES cell rescue experiment. For each construct that was transfected, the number of colonies analyzed (left column) and of colonies with full Cre-mediated Ctcfdelneo allele deletion (middle column) are shown. Colonies were analyzed by various methods to verify the intended deletion. The final conclusion regarding functional CTCF substitution is depicted in the right hand column. Of note, we only obtained one ES cell line expressing del1. Sequencing revealed that it contained a deletion of one extra amino acid $(\mathrm{K})$ at the $\mathrm{N}$-terminus of the originally intended del1 stretch (which begins with TFQ), and that the D residue situated at the C-terminus of the intended deletion stretch (which ends with HTD) was not deleted but was instead mutated into an E. Both changes are indicated in Fig. 1a.

d) Log2 fold change in CTCF ChIP-Seq signal in del8- versus GFP-CTCF-expressing ES cells.

e) Motif analysis of DNA binding by CTCF ZF mutants compared to GFP-CTCF (top row). Sites with more than 2-fold increased binding were included (number of sites are between brackets). Core (C), upstream (U), and downstream (D) motifs are indicated. Arrows point to $U$ motif nucleotides. These are shifted towards the core in the del8 mutant. Asterisks point to $\mathrm{GC}$ residues appearing in all mutant motifs.

Extended data Fig. 2. Interphase localization and behaviour of GFP-tagged CTCF proteins.

a) Proliferation assay of wild type (WT) ES cells, GFP-CTCF-expressing ES cells, and ES cell lines expressing the indicated GFP-tagged mutant proteins. We seeded 100000 cells at time 0 and replated 100000 cells after 48 hours for two more times. hr: hours. Experiments were done in triplicate, shown are averages \pm SD. The inset shows cell cycle doubling time of GFP-CTCF- and del8-expressing ES cells (GC and d8, respectively) with averages \pm SD.

b) Cell cycle analysis. GFP-CTCF- and del8-expressing ES cells were fixed, stained with propidium iodide (PI), and analyzed by fluorescence activated cell sorting (FACS). PI signal intensity is shown on the horizontal axis. Two FACS experiments were performed, one is shown, with percentages of the different cell cycle fractions indicated. Average values of the cell cycle fractions in the two experiments are shown below the graph. 
c) Intracellular distribution of GFP-tagged del9 (green) in ES cells incubated with SiRHoechst (red). Scale bar : 8 micron.

d) Fluorescence intensities in the indicated ES cell lines, measured by FACS, which reveals mean fluorescence intensity (MFI) per cell. WT: wild type ES cells, GC: GFP-CTCFexpressing ES cells, 1: del1-, 8: del8-, 9: del9-, 10: del10-, 11: del11-expressing ES cells.

e) Fluorescence intensity comparison of GFP-CTCF, del8, and EB3-GFP. To estimate the average number of expressed GFP-CTCF or del8 molecules in ES cell nuclei (see right hand panel for still image of an ES cell colony expressing GFP-CTCF; scale bar : 12 micron), we determined the fluorescence intensity of various dilutions of a purified GFP-tagged protein (EB3-GFP) of known concentration, and generated a calibration curve (lower graph, regression line (red) and function are shown). We next used the same microscope settings to measure GFP-CTCF (GC) and del8 (d8) average fluorescence intensity (FI) per nuclear area in arbitrary units (a.u., left hand graph). We then compared intensities of EB3-GFP to that of GFP-CTCF or del8. Based on this analysis we calculated that GFP-CTCF has an average nuclear concentration of $290 \mathrm{nM}$ and del8 of $190 \mathrm{nM}$.

Extended data Fig. 3. Behaviour of GFP-CTCF- and del8-expressing cell lines.

a) Proliferation assay of a GFP-CTCF-expressing ES cell line (GC), and of three ES cell lines expressing del8 (d8.1, d8.2, d8.3). We seeded 100000 cells at day 0 and counted cells after 1,2 , or 3 days. Experiments were done in triplicate, shown are averages \pm SD. All three del8 lines grow poorly in comparison to the $\mathrm{GC}$ line.

b) Intracellular distribution of GFP-CTCF (GC) and of del8 in three independently isolated ES cell lines expressing (d8.1, d8.2, d8.3). Asterisk depicts a nuclear area of low fluorescence in the GC line, representing the nucleolus. This area is not visible in the del8 lines. Scale bar : 8 micron.

c) Fluorescence intensities in the indicated ES cell lines, measured by confocal microscopy, which reveals average fluorescence intensity (FI) in arbitrary units (a.u.). GC: GFP-CTCFexpressing ES cells ( $n=183$ ), d8: del8-expressing ES cells (d8.1: $n=322$, d8.2: $n=159$, d8.3: $\mathrm{n}=167$ ). The average $\mathrm{FI}$ of GFP-CTCF- and del8-expressing ES cells is similar, indicating similar nuclear concentrations.

d) Fluorescence recovery after photobleaching (FRAP) experiments in ES cells expressing GFP-CTCF (GC) or del8 (d8.1, d8.2, d8.3). Average values of two FRAP experiments are shown (GC: 22 cells measured, d8.1: 32 cells measured, d8.2: 24 cells measured, d8.3: 27 cells measured). For clarity we did not indicate SEMs. Note that $\mathrm{d} 8.2$ is the line used in many 
of the other experiments presented in this manuscript whereas the GC line is a second independently isolated line expressing GFP-CTCF. Recovery of the del8 mutant proteins is very similar in the three lines, in part because the nuclear concentrations (as examined in panel (c)) are similar. Recovery of GFP-CTCF in the second GC line is also very similar to that of GFP-CTCF in the first GC line shown in Fig.2.

\section{Extended data Fig. 4. Prophase localization of GFP-CTCF.}

ES cells expressing GFP-CTCF (green) were incubated with SiR-Hoechst (red) and imaged during mitosis. Still images are shown of ES cells in prophase. Note the depletion of CTCF signal on areas of condensed chromatin, characterized by higher SiR-Hoechst signal. Scale bar : 5 micron.

Extended data Fig. 5. Analysis of 3D genome topology in ES cells expressing GFP-CTCF or the del8 mutant.

a) Principal component analysis (PCA) of the top 1000 genes with highest standard deviation in ES cells expressing del8 (d8) or GFP-CTCF (GC). We performed RNA-Seq on three biological replicate cultures of three independently isolated d8 lines (d8.1, d8.2, d8.3) and two GC lines (GC1, GC2). One RNA-Seq sample of clone d8.3 show poor quality metrics and was therefore excluded.

b) Reproducibility scores (based on spectral decomposition; on a 0 to 1 scale, see Methods for details) across in-situ Hi-C matrices between the indicated pairwise comparisons of conditions. Note high reproducibility scores $(>0.7)$ amongst biological replicates, which significantly drop (<0.4) when correlating in-situ Hi-C datasets from GC and d8-expressing cells.

c) Distance decay curves of chromosome-wide interactions for wildtype and del8 expressing ES cells.

d) Absolute PC1 values of genome-wide A and B compartment regions (100kb bins) that switch compartment when comparing GC and d8-expressing cells.

e) Change in mRNA expression levels as measured by RNA-Seq for genes located in genomic bins that switch compartment when comparing GC- and del8-expressing cells.

f) Absolute PC1 values of genome-wide A and B compartment regions (100kb bins) for untreated and CTCF-depleted cells. Data were obtained from ${ }^{2}$. Pie chart indicated genomic regions $(100 \mathrm{~kb}$ bin size) that switch compartment $(4.1 \%$ of the genome) due to CTCF depletion. 
g) 2D meta-plot quantifying inter-TAD interaction frequencies between superenhancers in untreated and CTCF-depleted cells².

Extended data Fig. 6. Aspects of TAD organization in ES cells expressing GFP-CTCF or del8. a) Percentage of TAD borders that are conserved between GFP-CTCF (GC) and del8 (d8) expressing ES cells. Conservation is further increased with larger window sizes ( $1 \mathrm{bin}=50 \mathrm{~kb}$ ).

b) Boxplot depicting the distribution of TAD sizes in GC- and del8-expressing cells.

c) Log2 fold change (FC) in insulation scores (IS) at TAD borders called in GC-expressing cells. Borders were categorized based on $>20 \%$ increase in IS ('gain'), $>20 \%$ decrease in IS ('loss') or $<20 \%$ change in IS ('stable') in del8-expressing cells (d8) as compared to GCexpressing cells.

d) Spie chart depicting the overlap between $A / B$ compartment junctions and the three TAD border categories specified in panel c. Outward or inward extension of the spie chart components indicates positive or negative enrichment, respectively. Dashed circles indicates a relative enrichment of 1 . Table shows border and overlap statistics.

e) Correlation plot showing for each TAD border called in GC-expressing ES cells how the change in IS as a consequence of ZF8 deletion (GC vs d8) compares to the change in IS measured after $48 \mathrm{~h}$ of acute CTCF depletion (untreated vs. auxin; data obtained from ${ }^{2}$ ).

f) Differential IS at all TAD borders detected in GC cells after ZF8 deletion (left) or CTCF depletion ${ }^{2}$ (right).

g) IS changes (GC-d8 differential values) for TAD borders bound or not bound by CTCF in GC cells.

h) Spie chart depicting the overlap between SMC1 loop anchors (top) or published ES cell loops (bottom) and the three TAD border categories specified in panel c. Outward or inward extension of the spie chart components indicates positive or negative enrichment, respectively. Dashed circles indicates a relative enrichment of 1 .

i) Meta-plot of Hi-C interaction signals for a set of validated ES cell loops ${ }^{3}$ in GC- and d8expressing ES cells.

j) Sex chromosome analysis of ES cells expressing GFP-CTCF (GC) or del8 (d8). The left upper panel displays a PCR on DNA with sex chromosome-specific primers. Controls are genomic DNA from male $(\mathrm{m})$ or female $(\mathrm{f})$ mice. The GC and d8 lines do not contain a $y-$ chromosome. Right hand panels show representative images of a fluorescent in situ hybridization (FISH) on ES cells using a fluorescent $x$-chromosome probe (red). DAPI is shown in green. Based on the FISH experiments we calculated the number of $x$ - 
chromosomes present in GC and d8 ES cells. As shown in the bar plot on the bottom left ( 5 experiments $\pm S D$ ) the number of $x$-chromosomes varies between 1 and 2 .

k) Differential Hi-C interaction map of chromosome X. CTCF peak calls in both wildtype and del8 expressing cells are indicated on the left. Note the strongly increased interaction frequencies across a large domain encompassing the Dxz4 locus in del8 (d8) expressing cells.

I) Violin plot of gene expression output (sum of all RPKM values from genes within a border) by the three TAD border categories in wildtype and del8 expressing cells.

m) CTCF binding site distribution with respect to distance to nearest TSS. Left graph: all CTCF binding sites, right graph: UC-containing sites. The inset shows a more detailed distribution in a $5 \mathrm{~kb}$ window surrounding the TSS.

Extended data Fig. 7. Transcriptome analysis of ES cells expressing the de/8 CTCF mutant. a) Venn diagram ${ }^{4}$ showing the overlap in differentially expressed genes (DEGs) between wildtype (GC) versus del8 (d8) expressing ES cells (this study) or those found when comparing control versus CTCF-depleted (48h auxin-treated) cells ${ }^{2}$. 4-way Venn diagram on the right includes directionality of gene expression changes.

b) Venn diagram showing the overlap in DEGs between wildtype (GC) versus del8 (d8) expressing ES cells (this study) or those found when comparing control versus RNA-binding region deficient CTCF (RBRi) expressing cells 5 . 4-way Venn diagram on the right includes directionality of gene expression changes.

c) Selected biological pathways associated with downregulated (top) or upregulated (bottom) genes in del8-expressing cells.

Extended data Fig. 8. Gene expression and DNA methylation changes in ES cells expressing the del8 CTCF mutant.

a) Immunofluorescence analysis of Dnmt3a levels in ES cells expressing GFP-CTCF (GC) or del8. Left: cells were stained with anti-Dnmt3a antibodies (red). DAPI (blue) was used to visualize the nucleus. We enhanced brightness and contrast in the panels to the same extent to visualize the low Dnmt3a signal in GC cells. This leads to an overexposed image in the del8 panel. Right: quantification of fluorescent Dnmt3a signal reveals significant differences in GC and del8-expressing ES cells (two-sided t-test).

b) Western blot analysis of cell lysates from two ES cell lines expressing GFP-CTCF (GC1, GC2) and three ES cell lines expressing del8 (d8.1, d8.2, d8.3). Western blots were probed 
with antibodies against Dnmt3a (upper) or tubulin (lower). "L" and "S" depict long and short Dnmt3a isoforms, respectively. We quantified Dnmt3a levels relative to the GC2 line by measuring the intensity of Dnmt3a signal over that of tubulin. Data are shown below the lanes. Consistent with RNA-Seq results, all the del8 lines express higher amounts of Dnmt3a as compared to the GC lines.

c) Expression level of Dnmt3b in GFP-CTCF (GC) and del8 (d8) expressing ES cells. Data were taken from the RNA-Seq results shown in Extended Data Table I. ${ }^{* *} \mathrm{P}<0.001$ (Wald test corrected for multiple testing)

d) Immunofluorescence analysis of ES cells expressing GFP-CTCF (GC) or del8. Left: cells were stained with anti-5mC antibodies (red). Right : quantification of fluorescent $5 \mathrm{mC}$ signal reveals significant differences in GC and del8-expressing ES cells (two-sided t-test).

e) Principal component analysis (PCA) of differentially methylated regions (DMRs) showing $>2$ fold change in methylation in ES cells expressing del8 (d8) or GFP-CTCF (GC). We performed MedSeq in duplicate for three independently isolated d8 lines (d8.1, d8.2, d8.3) and two GC lines (GC1, GC2).

f) Genomic localization of differentially methylated regions (DMRs) divided in hypermethylated (top, $n=290$ ) or hypo-methylated (bottom, $n=82$ ) regions in del8-expressing cells as compared to wildtype ES cells.

g) Correlation scatter plot comparing changes in DNA methylation (log2 d8-GC, y-axis) and changes in CTCF ChIP-Seq signal (log2 d8-GC, x-axis) for 87 DMRs (>3 fold change in DNA methylation) that contain a CTCF binding site.

Extended data Movie 1. GFP-CTCF behaviour during mitosis.

ES cells expressing GFP-CTCF were imaged at a resolution of $100 \mathrm{~nm} /$ pixel using a Leica confocal microscope. In total we acquired 424 images at a frame rate of 1 image per 5 seconds. Note that for this particular experiment several independent short time lapse experiments were performed one after the other and at the end movies were stitched together.

Extended data Table 1. Differential gene expression analysis using RNA-Seq data from GFPCTCF and del8-expressing ES cells.

Extended data Table 2. HiC data statistics. 


\section{References}

1. Hashimoto, H., Wang, D., Horton, J.R., Zhang, X., Corces, V.G. \& Cheng, X. Structural Basis for the Versatile and Methylation-Dependent Binding of CTCF to DNA. Mol Cell 66, 711-720 e3 (2017).

2. Nora, E.P., Goloborodko, A., Valton, A.L., Gibcus, J.H., Uebersohn, A., Abdennur, N., Dekker, J., Mirny, L.A. \& Bruneau, B.G. Targeted Degradation of CTCF Decouples Local Insulation of Chromosome Domains from Genomic Compartmentalization. Cell 169, 930-944 e22 (2017).

3. Krijger, P.H., Di Stefano, B., de Wit, E., Limone, F., van Oevelen, C., de Laat, W. \& Graf, T. Cell-of-OriginSpecific 3D Genome Structure Acquired during Somatic Cell Reprogramming. Cell Stem Cell 18, 597-610 (2016).

4. Bardou, P., Mariette, J., Escudie, F., Djemiel, C. \& Klopp, C. jvenn: an interactive Venn diagram viewer. BMC Bioinformatics 15, 293 (2014).

5. Hansen, A.S., Hsieh, T.S., Cattoglio, C., Pustova, I., Saldana-Meyer, R., Reinberg, D., Darzacq, X. \& Tjian, R. Distinct Classes of Chromatin Loops Revealed by Deletion of an RNA-Binding Region in CTCF. Mol Cell (2019). 
a

1

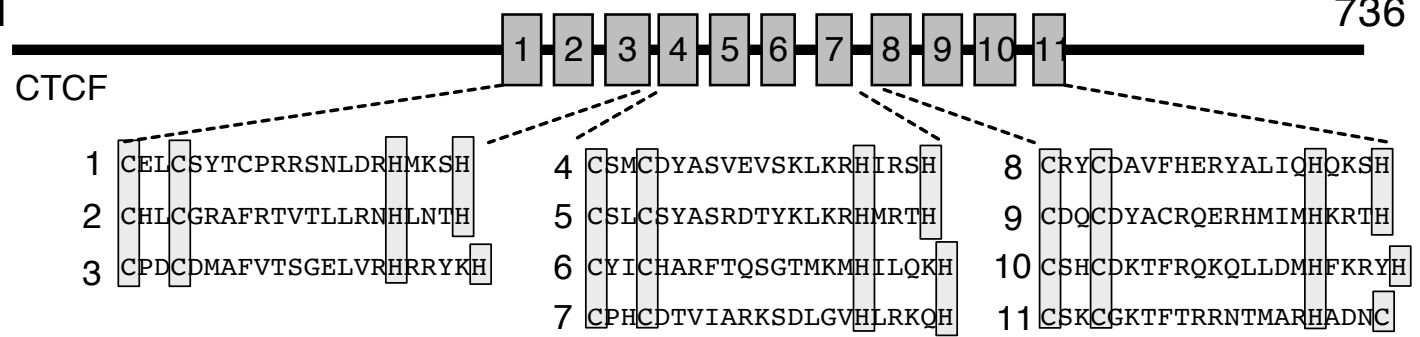

b

C

$\begin{array}{lccc}\text { CTCF construct } & \begin{array}{c}\text { Number of } \\ \text { colonies } \\ \text { analyzed }\end{array} & \begin{array}{c}\text { Number of } \\ \text { colonies with } \\ \text { full deletion }\end{array} & \begin{array}{c}\text { CTCF } \\ \text { substitution }\end{array} \\ \text { GFP-CTCF } & 42 & 40 & \text { Yes } \\ \text { GFP-CTCF-del1 } & 16 & 1 & \text { Yes } \\ \text { GFP-CTCF-del2 } & 4 & 0 & \text { No } \\ \text { GFP-CTCF-del3 } & 9 & 0 & \text { No } \\ \text { GFP-CTCF-del4 } & 6 & 0 & \text { No } \\ \text { GFP-CTCF-del5 } & 7 & 0 & \text { No } \\ \text { GFP-CTCF-del6 } & 5 & 0 & \text { No } \\ \text { GFP-CTCF-del7 } & 5 & 2 & \text { No } \\ \text { GFP-CTCF-del8 } & 11 & 9 & \text { Yes } \\ \text { GFP-CTCF-del9 } & 6 & 5 & \text { Yes } \\ \text { GFP-CTCF-del10 } & 12 & 10 & \text { Yes } \\ \text { GFP-CTCF-del11 } & 8 & 6 & \text { Yes }\end{array}$

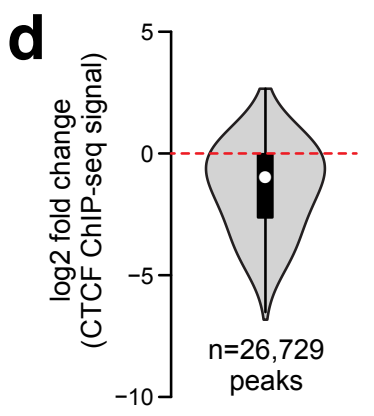

e
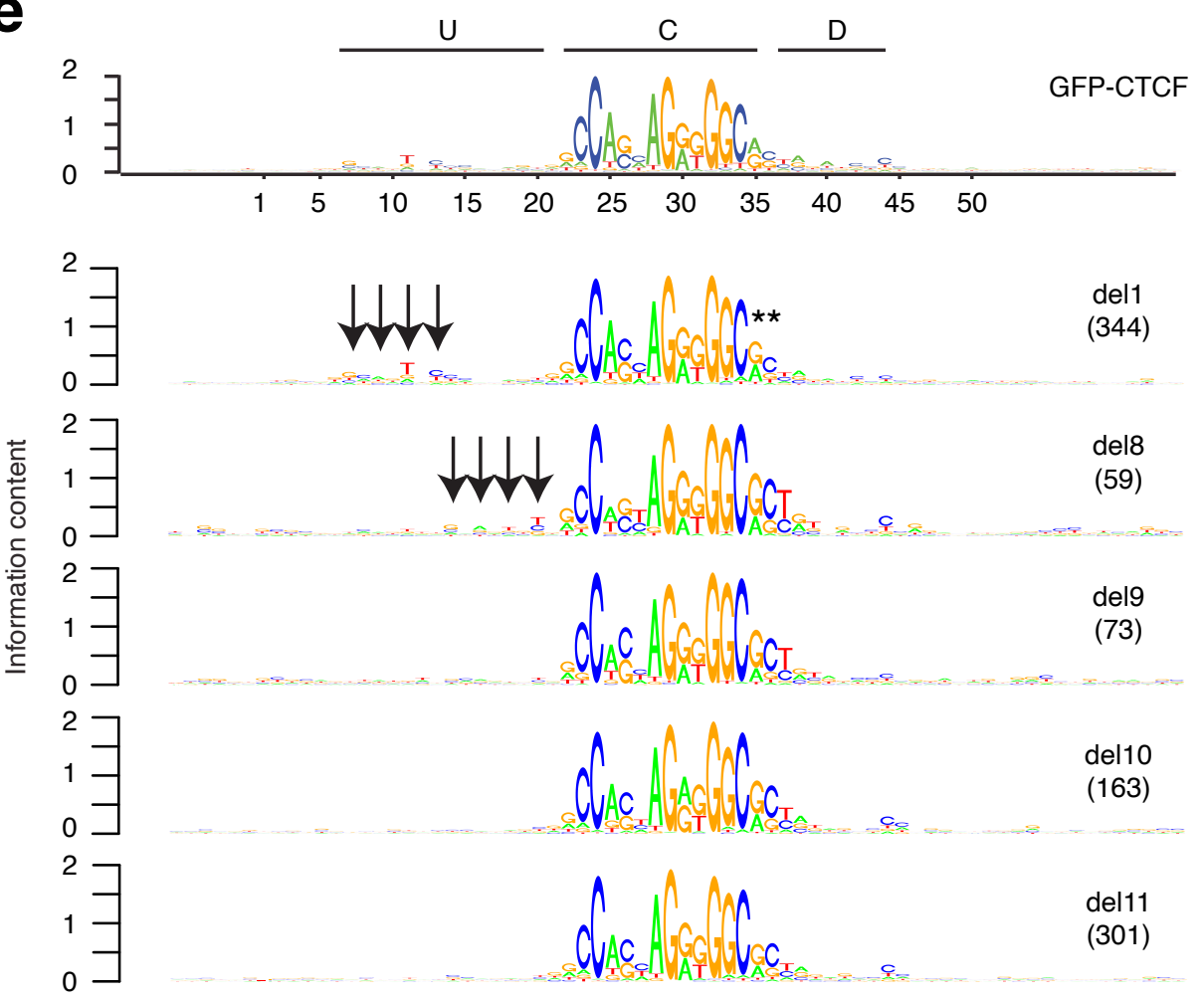
a

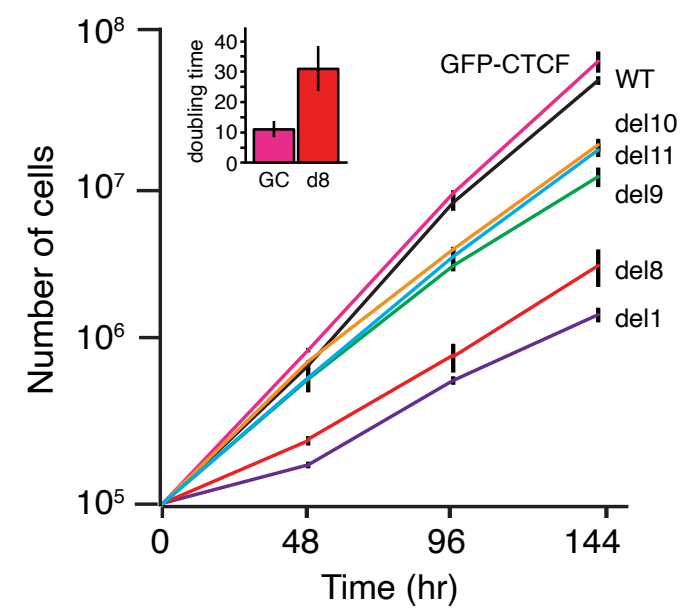

b
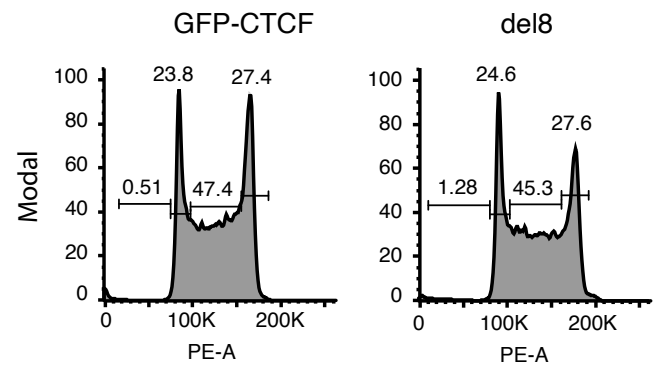

\begin{tabular}{rcccc} 
& apoptotic & G1 & S-phase & G2/m \\
\hline GFP-CTCF & 0.54 & 24.4 & 45 & 29.1 \\
del8 & 1.5 & 25.0 & 47 & 25.3
\end{tabular}

C

GFP
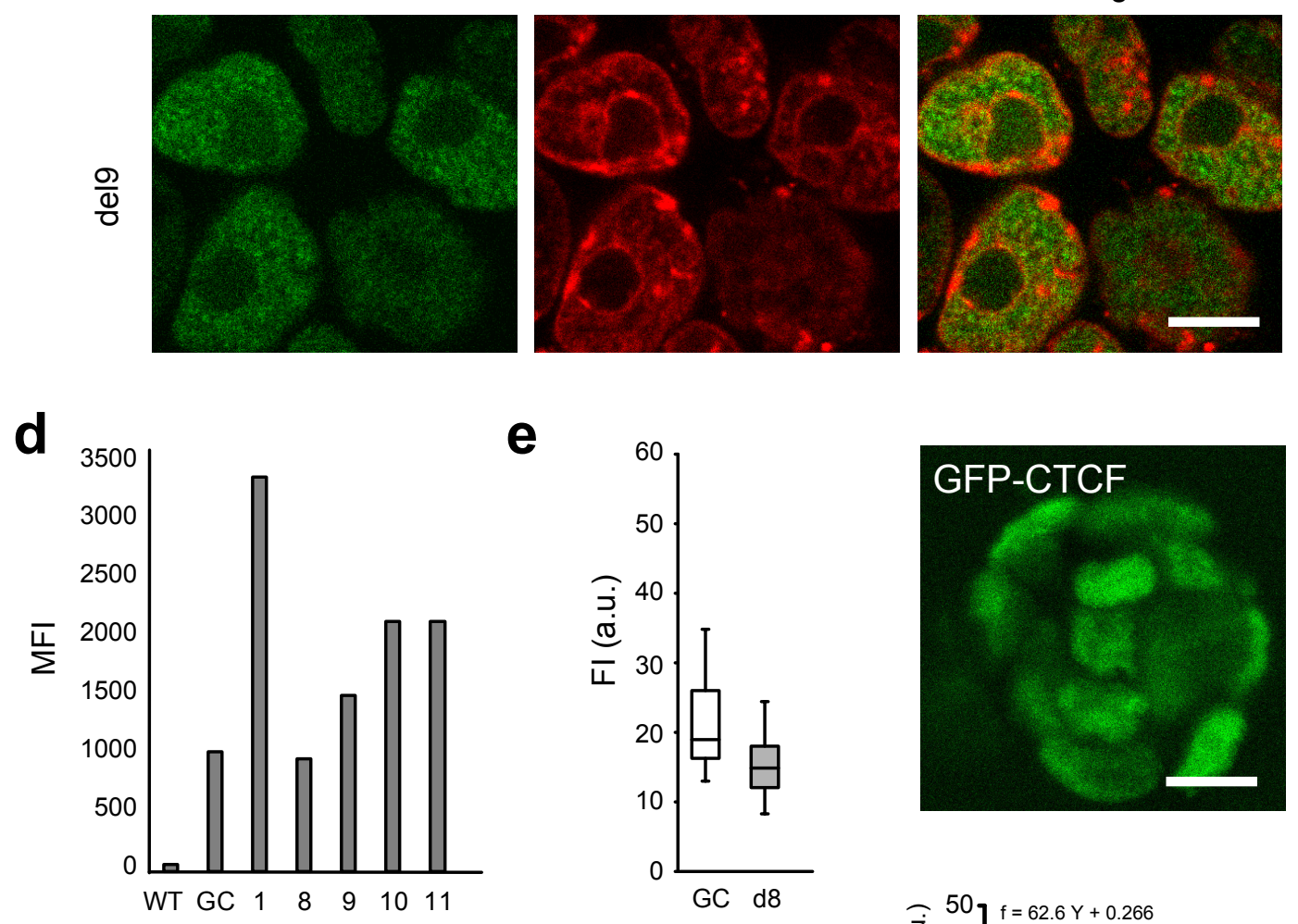

e

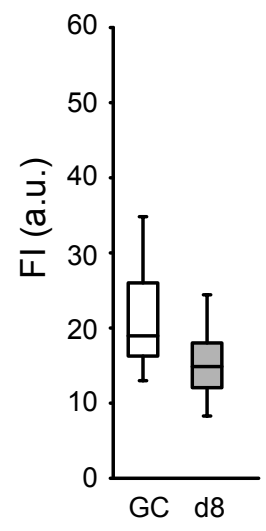

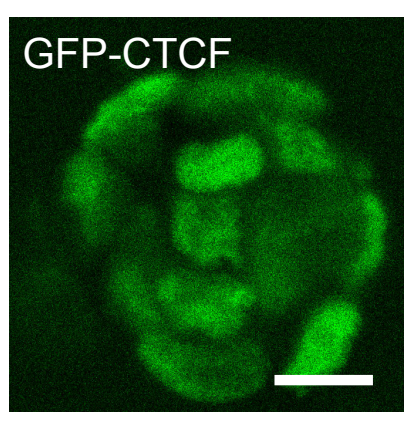

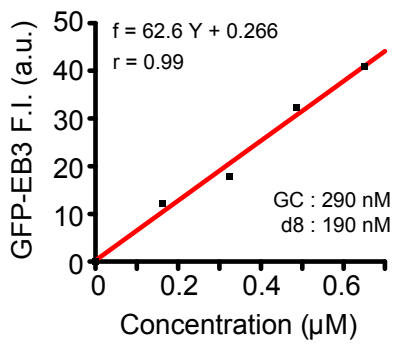


a

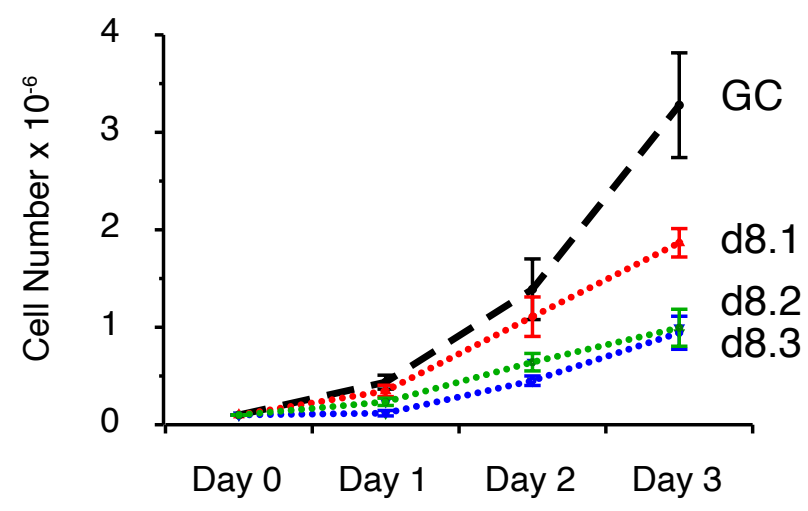

b
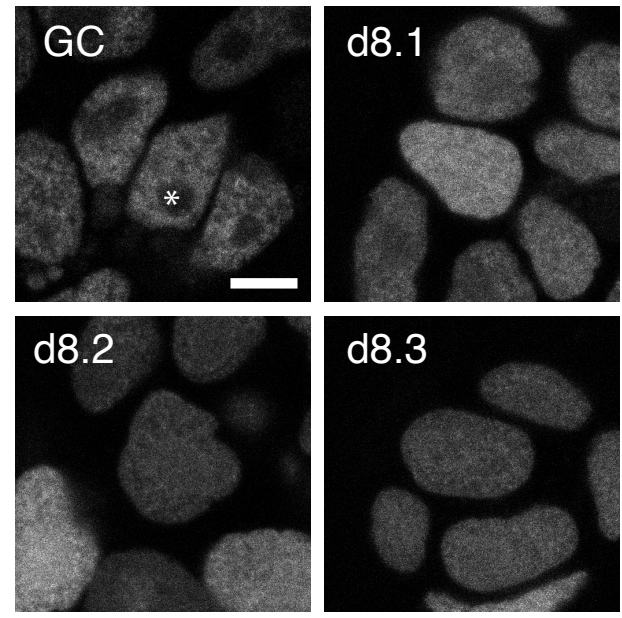

C
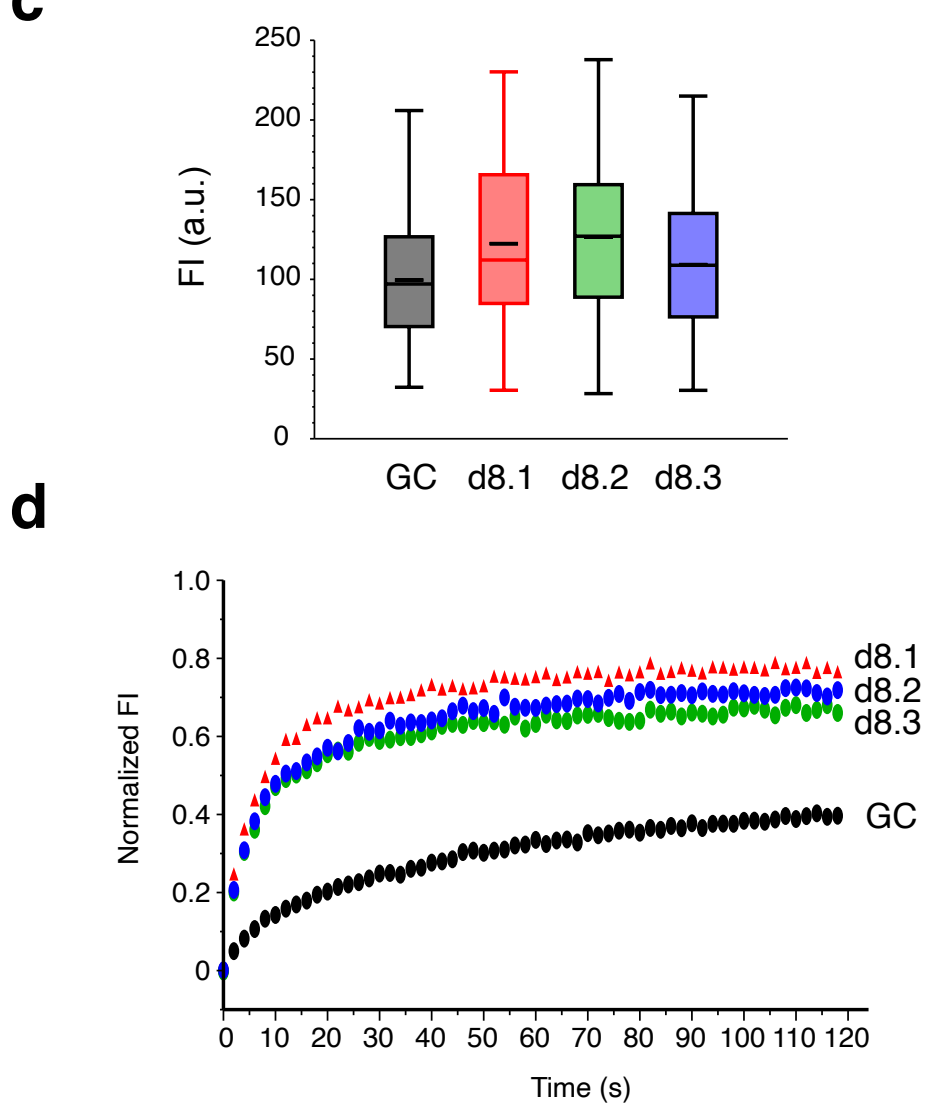

Extended data Figure 3 - Soochit 


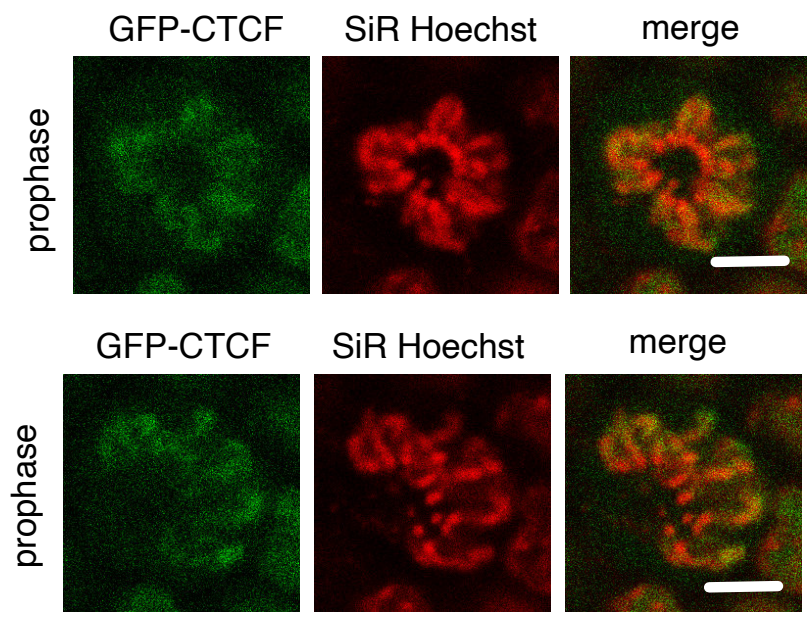


a

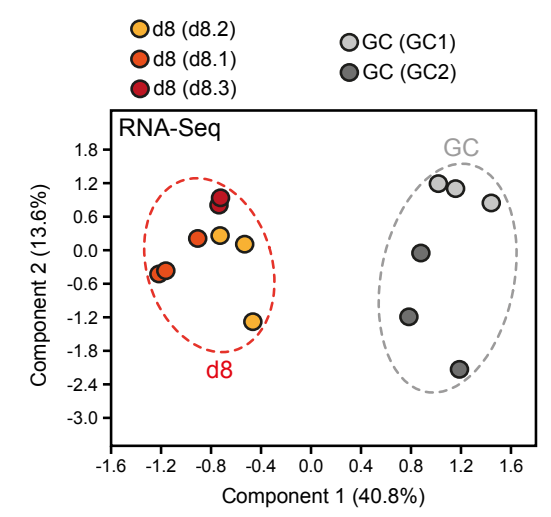

b

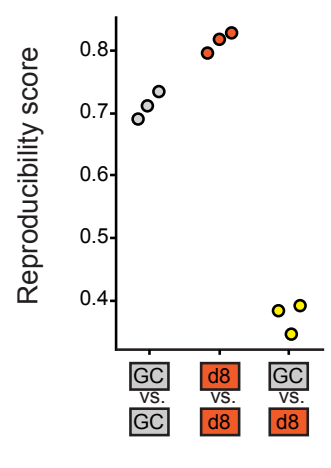

C

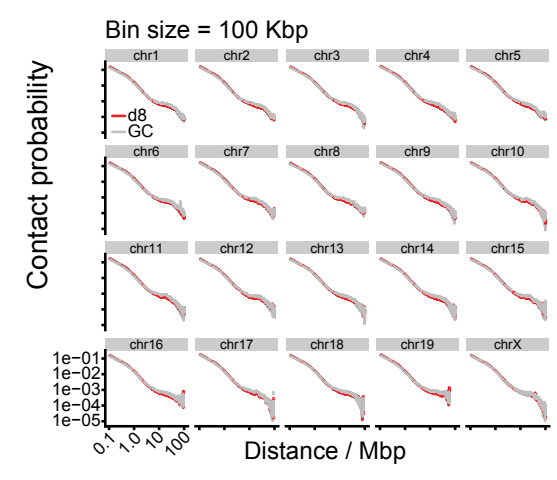

d

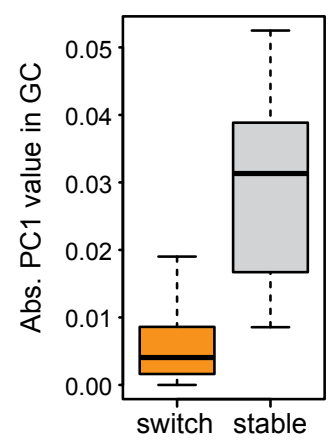

e

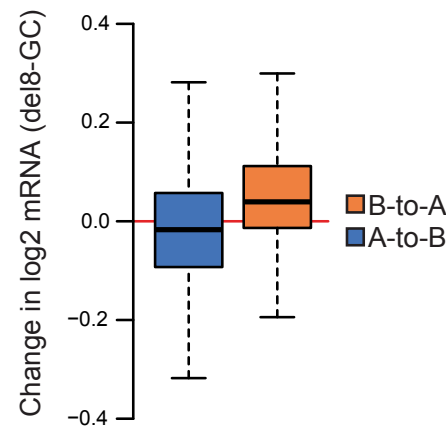

f

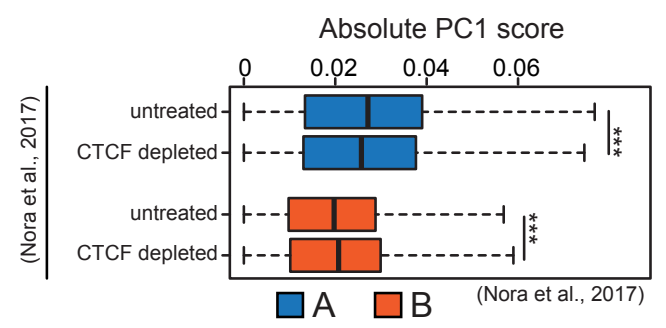

switching bins

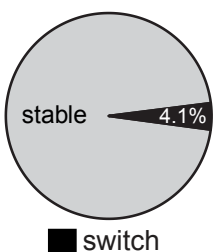

(Nora et al., 2017) g Long range SE interactions $(5-10 \mathrm{Mb})$

untreated CTCF depleted

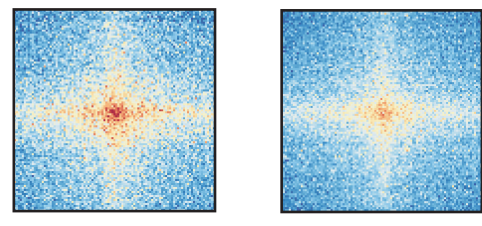

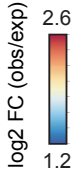

(Nora et al., 2017) 
a

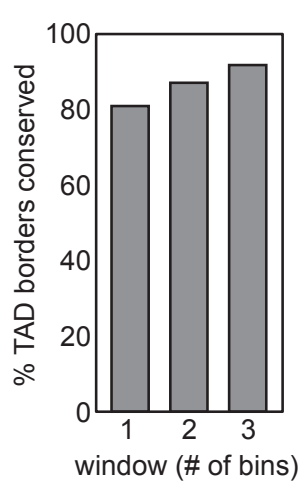

e

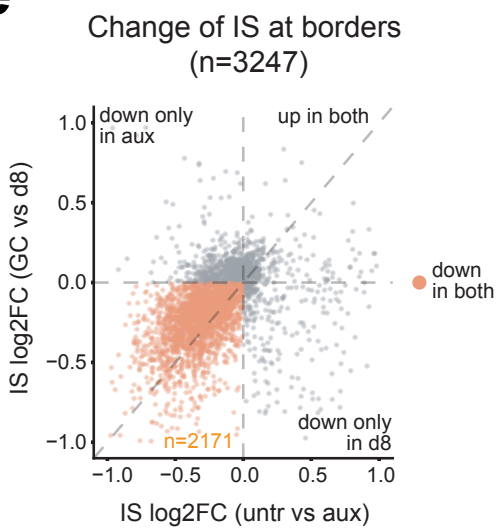

i

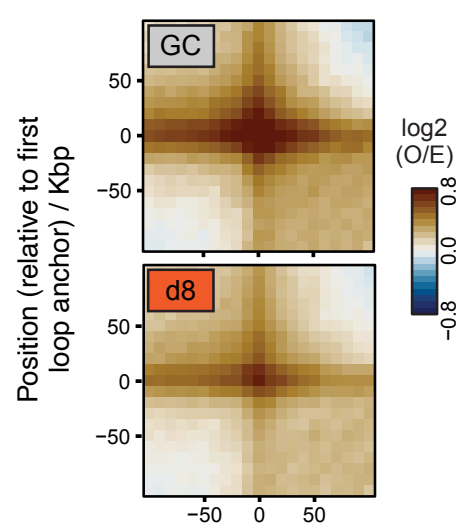

I

expression at borders

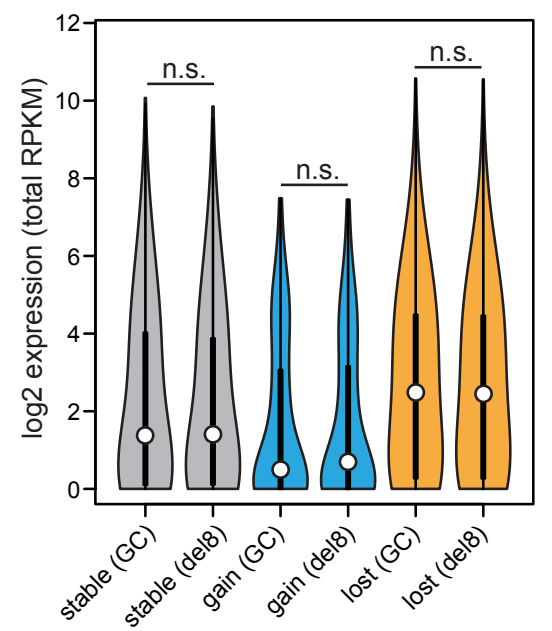

C

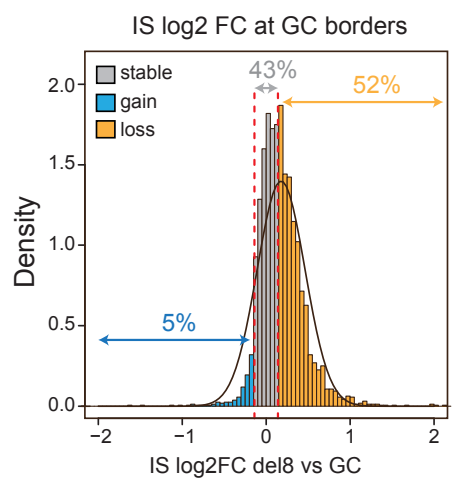

g TAD borders
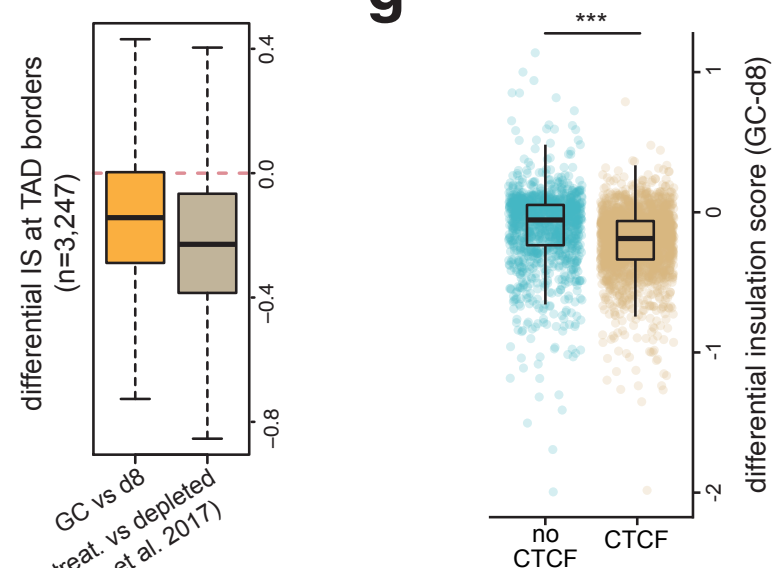

d

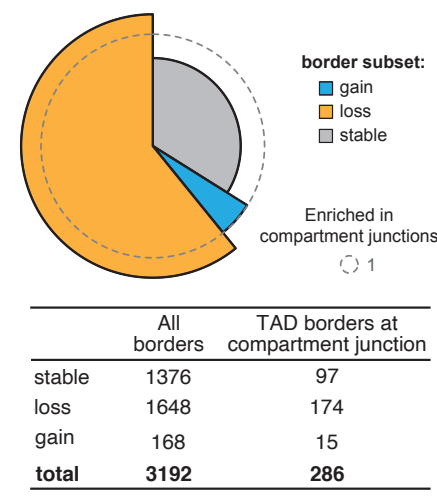

h

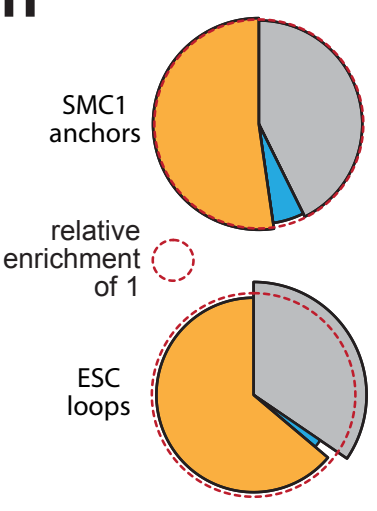

j

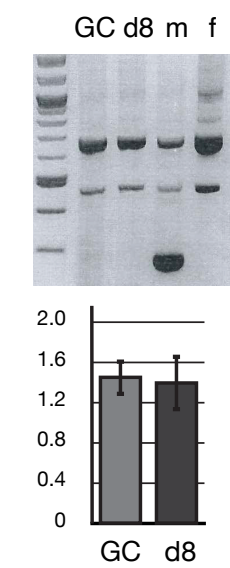

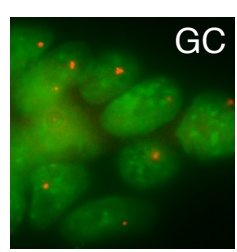

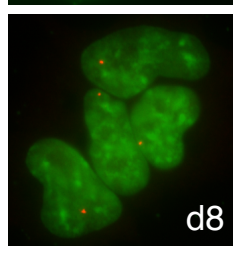

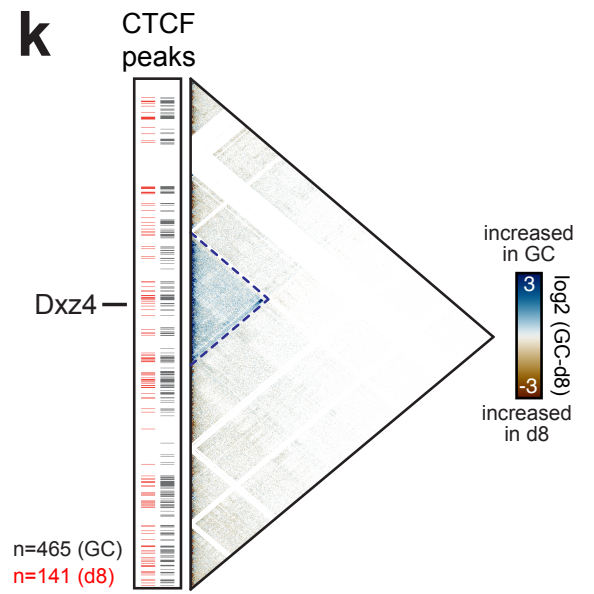

m

All CTCF

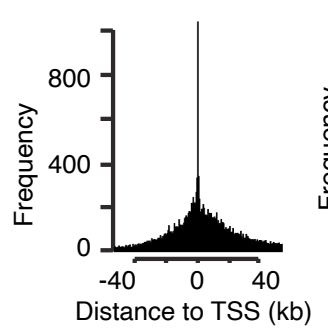

CTCF UC

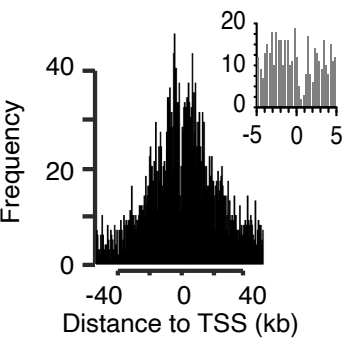




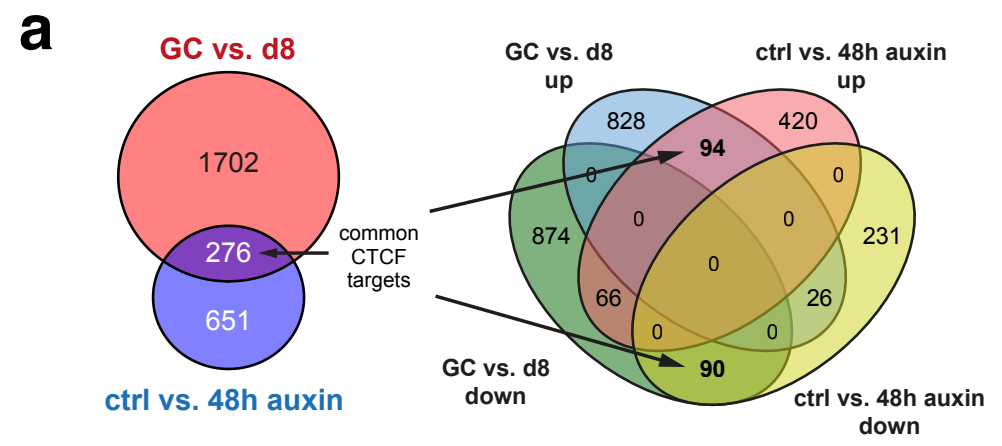

b

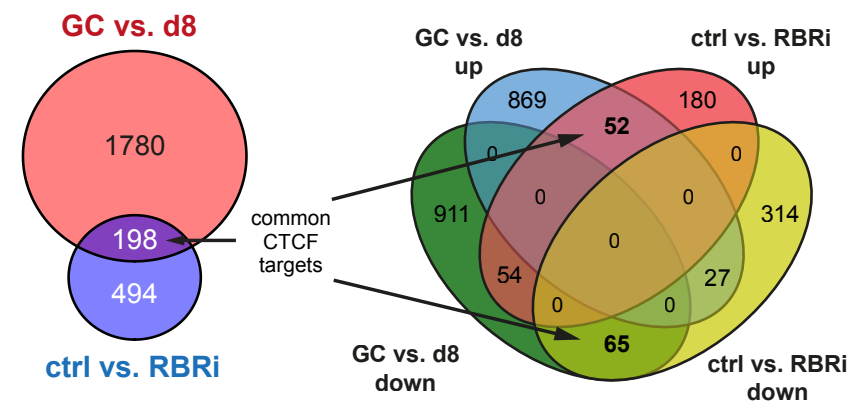

C
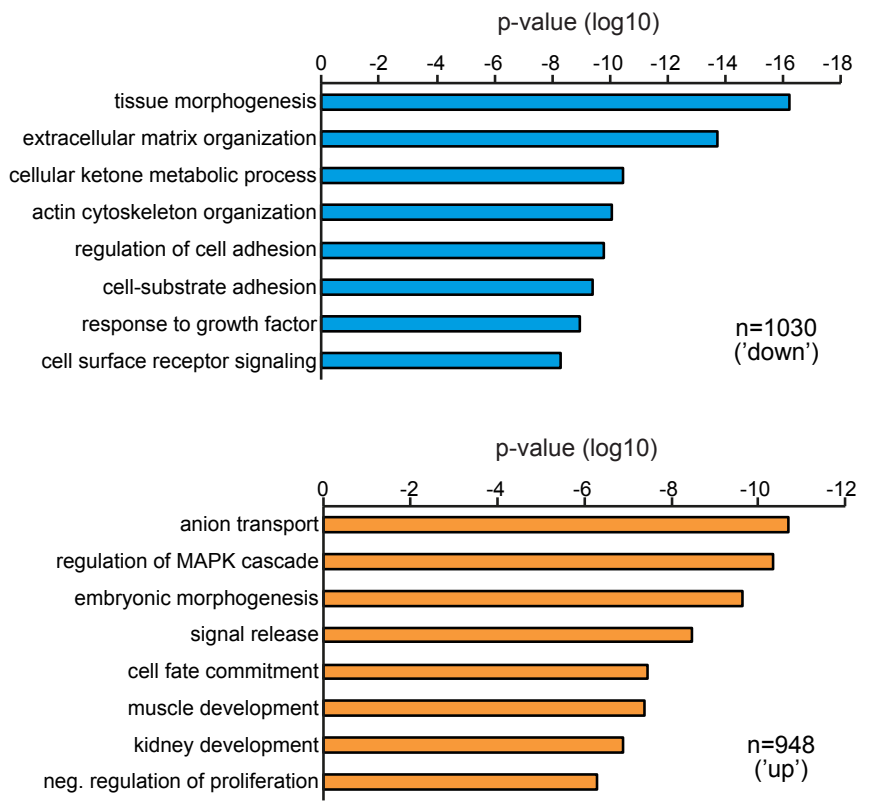
a
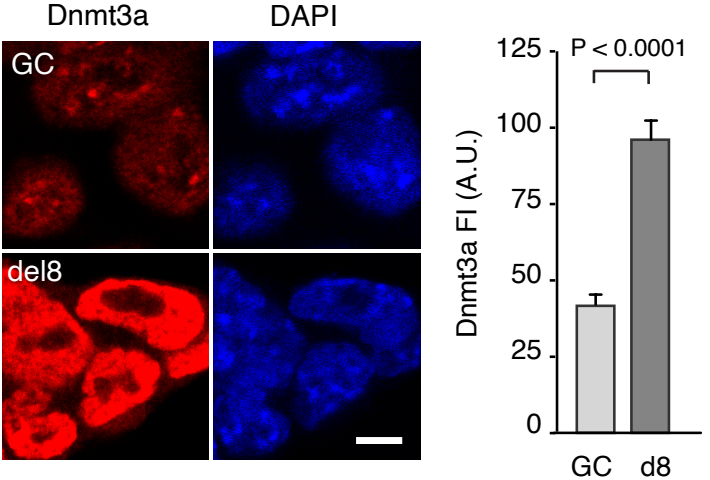

C

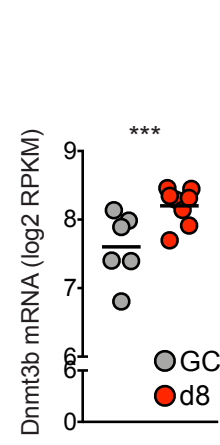

d $5 \mathrm{mC}$

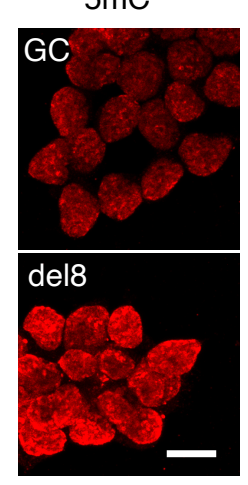

b

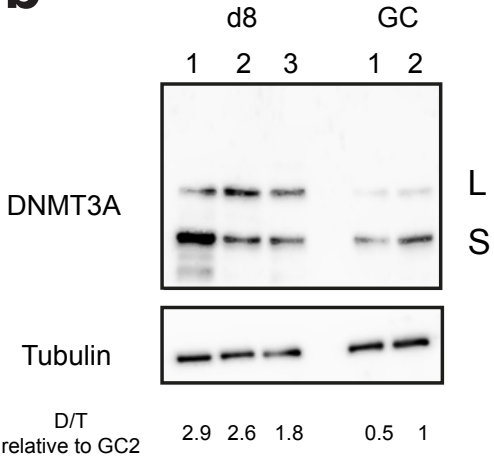

e

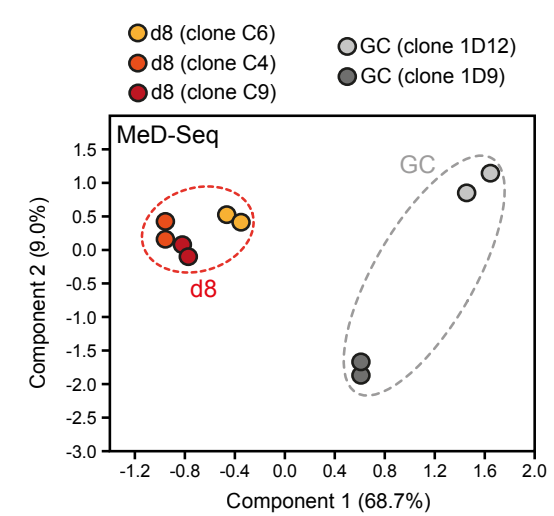

f

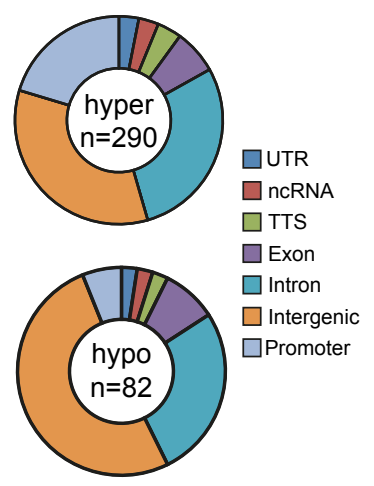

g

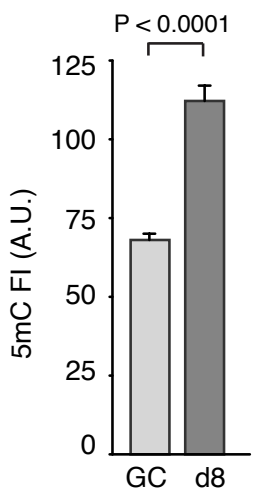

$r=-0.0006$ $\mathrm{P}=0.95$

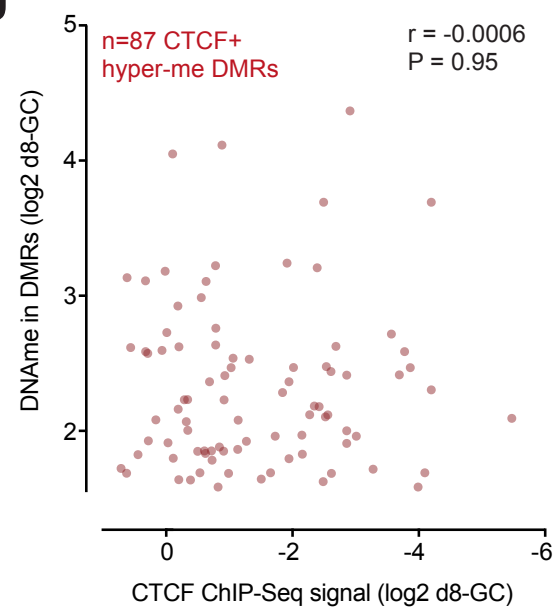




\section{Online Methods}

Generation of GFP-CTCF-expressing ES cells

To inactivate a given ZF, one can either choose to mutate critical residues (e.g. His, Cys) in a ZF, which are important for Zinc coordination, or delete the ZF. The advantage of the first approach is that, in a multi-ZF protein, the spacing of ZFs is maintained. The disadvantage is that unfolded protein domains with unpredictable properties are generated. We believe this disadvantage outweighs the advantage, which is why we chose to delete individual ZFs in CTCF. We generated lentiviral constructs expressing wild type GFP-CTCF or the different GFP-CTCF-ZF mutant proteins in combination with IRES-driven Cre recombinase ${ }^{1}$. CTCF expression was driven from a CAG promoter (CMV early enhancer/chicken $\beta$ actin). Ctcflox mice have been described previously ${ }^{2}$. We isolated Ctcflox/lox ES cells from homozygous mice and transiently treated these cells with Cre-recombinase to delete the neomycin resistance cassette; this yielded so-called Ctcf ${ }^{\text {delneo/delneo }}$ ES cells. These ES cells were infected with lentiviral particles in suspension for 4 hours and then plated. G418 selection was started one day after infection. Neomycin-resistant, GFP-positive clones were picked, expanded as described ${ }^{1}$, and analyzed by various assays to assess in detail the rescue by CTCF-ZF deletion mutants. All ES cell lines used in this study are available upon request.

\section{Characterization of ES cells}

ES cells were grown on plastic dishes coated with $0.2 \%$ gelatin (Merck) in the presence of ES cell medium containing Dulbecco's Modified Eagle Medium (DMEM) (Lonza), 15\% heat-inactivated fetal calf serum (FCS), Non Essential Amino Acids (Lonza), $100 \mathrm{U} \mathrm{\textrm {ml } ^ { - 1 }}$ penicillin and $100 \mathrm{mg} \mathrm{ml}^{-1}$ streptamycin, $0.1 \mathrm{mM} \beta-$ mercaptoethanol (Sigma) and $1000 \mathrm{U} / \mathrm{ml}$ leukaemia inhibitory factor (LIF). ES cells were cultured at $37^{\circ} \mathrm{C}$ with $5 \% \quad \mathrm{CO}_{2}$ levels. To measure $\mathrm{ES}$ cell growth and proliferation, 100,000 ES cells were plated in a 6-well dish. After 48 hours ES cells were harvested, counted and 100,000 cells were re-plated, which was repeated 3 times (144 hr). Alternatively, 100,000 ES cells were plated in a 6-well dish and cells were counted after 1,2 , and 3 days.

Cellular analysis using flow cytometry 
To measure fluorescence intensities in the ES cell lines expressing GFP-CTCF (wild type or mutant) we harvested ES cells and analyzed them by fluorescence activated cell sorting (FACS) as previously described ${ }^{1}$. FACS experiments were also carried out to analyze cell cycle phase distributions of GFP-CTCF and del8-expressing cell lines. For this analysis cells were collected, washed once in PBS, resuspended gently in 0.8 $\mathrm{ml}$ of PBS and transferred to a FACS tube. Ice-cold methanol $(2.2 \mathrm{ml})$ was added while vortexing to fix the cells. Cells were incubated for one hour at $4^{\circ} \mathrm{C}$ or at $-20^{\circ} \mathrm{C}$ for longer. After fixation, cells were washed once with PBS and resuspended in $0.5 \mathrm{ml} \mathrm{PI-}$ Buffer per 1 million cells (50ug/ml propidium iodide (PI), $10 \mathrm{mM}$ Tris- $\mathrm{HCl}$ pH 7.5, $5 \mathrm{mM}$ $\mathrm{MgCl}_{2}, 200 \mathrm{ug} / \mathrm{ml}$ RNase A). Tubes were incubated for 20 to $30 \mathrm{~min}$ at $37^{\circ} \mathrm{C}$ in order to digest RNA. Stainings were quantified on a BD LSR-Il flowcytometer and analyzed using FACSDiva software.

\section{Standard molecular biology methods}

Genomic DNA was isolated by incubating cells at $55^{\circ} \mathrm{C}$ on a rocking platform in buffer containing $50 \mathrm{mM}$ Tris- $\mathrm{HCl} \mathrm{pH}$ 8.0, 5 mM EDTA pH 8.0 and 0.5\% SDS, $0.3 \mathrm{mg} / \mathrm{ml}$ proteinase $\mathrm{K}$ and $0.01 \mathrm{mg} / \mathrm{ml}$ RNase. This incubation was followed by $\mathrm{NaCl}$ addition (to $1.2 \mathrm{M}$ ) and centrifugation. Isopropanol and $70 \%$ ethanol were used for DNA precipitation and washing, respectively, and pellets were dissolved in $10 \mathrm{mM}$ Tris and $1 \mathrm{mM}$ EDTA. Genomic DNA was either used for PCR (primers available on request), or for Southern blot analysis. In the latter case DNA was digested with Hind III and loaded on an agarose gel for size fractionation. Samples were blotted onto a Hybond $\mathrm{N}+$ membrane (Cytiva) and hybridized with radioactive probes using $\alpha^{32} \mathrm{P}$ dATP. Signals were detected with a Phosphor Imager.

PCR for sex chromosome analysis in GFP-CTCF- and del8-expressing ES cells was conducted after extraction of genomic DNA on the UBE-X and UBE-Y genes using the following primers ${ }^{3}$ :

Fwd: 5'- TGGTCTGGACCCAAACGCTGTCCACA - 3';

Rev: 5'-GGCAGCAGCCATCACATAATCCAGATG- 3'.

Total RNA isolation was performed with Trizol-chloroform extraction. After Trizol addition samples were incubated for 5 minutes at $30^{\circ} \mathrm{C}$. Chloroform was added and the aqueous phase was transferred after centrifugation. $100 \%$ ethanol was added and RNA was isolated using the RNeasy Mini Kit (Qiagen). 
Nuclear protein extracts from $10 \mathrm{~cm}^{2}$ cell culture dishes were generated by resuspending ES cells in $500 \mu \mathrm{l}$ buffer A (10 mM Hepes, $1.5 \mathrm{mM} \mathrm{MgCl}, 10 \mathrm{mM} \mathrm{KCl}$, and protease inhibitors (Complete, Roche)) followed by 10 minutes incubation on ice. Samples were centrifuged and nuclei were lysed by adding $250 \mu \mathrm{l}$ buffer C ( $420 \mathrm{mM}$ $\mathrm{NaCl}, 1.5 \mathrm{mM} \mathrm{MgCl}$, $200 \mathrm{mM}$ EDTA, $20 \mathrm{mM}$ Hepes hOH, 21.75\% glycerol and protease inhibitor) followed by 20 minutes incubation on ice. The supernatant was used for further assays.

For western blot analysis nuclear protein extracts were loaded on a SDS-PAGE gel. After electrophoresis proteins were transferred to PVDF membranes (Millipore) via semi-dry blotting (Bio-Rad, trans-blot SD, semi-dry transfer cell). Membranes were blocked in PBS containing 0.05\% Tween-20 and either 2\% BSA, or in 5\% Milk (Sigma). Antibody incubations were done in the same buffer. The following antibodies were used: GFP (rabbit home made 1:1000), CTCF (rat, KT70, Absea, 1:1000), UBF (1:1000), Dnmt3a (Abcam, ab13888, 1:1000), and tubulin (mouse, Thermo Fisher, $1: 1000)$.

\section{Standard fluorescence-based microscopy experiments}

ES cells expressing wild type or mutant CTCF were grown on glass coverslips until small- to medium-sized colonies appeared. All live imaging was done in normal culture medium. For fluorescence time lapse imaging, cells were examined on a Leica TSP5 confocal microscope as described previously, using different image acquisition times ${ }^{4}$. In some experiments SiR Hoechst ${ }^{5}$ was added approximately 30 minutes prior to the imaging experiment, in a concentration of $100 \mathrm{nM}$, as recommended by the supplier (Spirochrome).

For immunofluorescence experiments in fixed cells we used two fixation protocols. For Dnmt3a visualization cells were fixed in $4 \%$ paraformaldehyde in PBS for 20 minutes at room temperature, permeabilized in $0.15 \%$ Triton X-100 in PBS, blocked in $1 \%$ BSA in PBS $/ 0.05 \%$ Tween 20 , and stained with antibodies as described 6 . For $5 \mathrm{mC}$ visualization cells were fixed in methanol containing $1 \mathrm{mM}$ EGTA $\left(-20{ }^{\circ} \mathrm{C}\right.$ for 20 minutes), permeabilized in $0.15 \%$ Triton $\mathrm{X}-100$ in PBS, treated with $4 \mathrm{M}$ $\mathrm{HCl}$ for $10 \mathrm{~min}$, washed twice with water and then three times with PBS, blocked in $1 \%$ BSA in PBS/0.05\% Tween20 and incubated with antibodies. For these studies we used anti-Dnmt3a (Abcam, ab13888, 1:100) or anti-5mC (Diagenode, C15200081, 
1:500) antibodies. Coverslips with stained cells were examined using a Leica TSP5 confocal microscope.

To calculate the concentration of GFP-CTCF in ES cell nuclei we measured the fluorescence intensity of a known fluorescent protein, i.e. GFP-EB3, which was expressed as a His-tagged protein in bacteria and purified using Ni-NTA beads. We applied different dilutions of this protein to small glass sample chambers, assembled from clean microscope slides and coverslips, and pre-incubated with k-casein to block the glass surface, as described ${ }^{7}$. To generate a standard curve we measured GFPEB3 fluorescence inside the chamber. Measurements were performed in triplicate. Using the exact same microscope settings, we then measured fluorescence intensity of GFP-CTCF and of del8 in ES cells.

To estimate the volume of an ES cell nucleus we assumed an ellipsoid shape of 10 by 5 micron $^{8}$. This yields a volume of 1 picolitre. We also measured length (at the longest point) and width (at the widest point) of z-section images of 42 nuclei taken from fluorescence recovery after photobleaching experiments (see below). This yielded values of $6.2 \pm 1.2$ micron (longest point) and $3.2 \pm 0.94$ micron (widest point). Assuming an ellipsoid nucleus, this yielded a volume of approximately 250 femtolitres, four times smaller than published ${ }^{8}$. Assuming such volumes, our data suggest that 50,000-200,000 molecules of GFP-CTCF are present within an ES cell nucleus, similar to the amount of GFP-CTCF expressed from a knock-in allele ${ }^{9}$.

\section{Fluorescence recovery after photobleaching (FRAP)}

Fluorescence recovery after photobleaching (FRAP) experiments in interphase cells were performed in ES cells expressing GFP-tagged wild type or mutant CTCF proteins, on a Leica TSP5 confocal microscope, essentially as described ${ }^{4}$. In each ES cell colony we bleached nuclei, using circular regions of interest (ROIs) with a diameter of 3 micron. We measured fluorescence recovery in the bleached region, compensating for fluctuations in fluorescence intensity in the same nuclei as well as in non-bleached cells. For the interphase cell experiments, we performed two types of FRAP experiment. In the first (depicted in Fig.2b and Extended data Fig.3d ) we measured recovery for two minutes with image acquisition times of two seconds. In the second FRAP experiment (not shown) we measured the recovery of selected GFP. CTCF proteins for 10 minutes with image acquisition times of 5 seconds. We obtained similar data with the two approaches. 
FRAP experiments in mitotic cells were performed on a Nikon Eclipse Ti-E microscope fitted with a Spinning Disc unit (CSU-X1, Yokogawa), using a Nikon 60x oil objective. The microscope was equipped with a Nikon perfect focus system and QuantEM 512SC EMCCD camera (Photometrics). Images were projected onto the CCD chip of the camera at a magnification of $112 \mathrm{~nm} /$ pixel. A stage top heater (INUG2E-ZILCS, Tokai Hit) and lens heater were used to maintain cells at $37^{\circ} \mathrm{C}$ during imaging. Acquisition was performed using Metamorph 7.5 (Molecular Devices). Focus was maintained throughout imaging using the perfect focus system. Photobleaching was performed using the iLAS FRAP module (Roper Scientific) integrated into Metamorph, with the $491 \mathrm{~nm}$ laser set at 100\%. A circular ROI was selected and 30 repetitions of the laser were used to bleach the region for a fixed time of $270 \mathrm{~ms}$. Images were acquired with the laser set at $39 \%($ gain $=800)$ at 1 frame per 5 seconds (exposure time $500 \mathrm{~ms}$ ). A few images were acquired before the bleach and acquistion was continued for 3-5 minutes post-bleach.

\section{Monte Carlo simulations}

For the model-based analysis of the FRAP data, raw FRAP curves were normalized to pre-bleach values and the best fitting curve (by ordinary least squares) was picked from a large set of computer simulated FRAP curves in which three parameters representing mobility properties were varied: diffusion rate (ranging from 0.04 to 25 $\mu \mathrm{m}^{2} / \mathrm{s}$ ), stable immobile fraction (ranging from 0-90\%), and average time spent in stable immobile state (ranging from 0.1 to $300 \mathrm{~s}$ ). The Monte Carlo computer simulations used to generate FRAP curves for fitting were based on a model ${ }^{10}$ that simulates diffusion of molecules and binding to immobile elements (representing chromatin), in an ellipsoidal volume (representing the cell nucleus). Laser bleaching was simulated based on experimentally derived three-dimensional laser intensity profiles, which were used to determine the probability for each molecule to get bleached considering their 3D position. The size of the ellipsoid representing the cell nucleus was based on the experimentally derived size of nuclei and the precise used FRAP protocol was mimicked, including the time intervals between monitoring of the bleached area during image acquisition after bleaching. The simulation of the FRAP curve was then run using discrete time steps. Diffusion was simulated at each new time step $t+\Delta t$ by deriving the new positions $\left(x_{t+\Delta t}, y_{t+\Delta t}, z_{t+\Delta t}\right)$ of all mobile molecules 
from their current positions $\left(x_{t}, y_{t}, z_{t}\right)$ by $x_{t+\Delta t}=x_{t}+G\left(r_{1}\right), y_{t+\Delta t}=y_{t}+G\left(r_{2}\right)$, and $z_{t+\Delta t}=z_{t}+G\left(r_{3}\right)$, where $r_{i}$ is a random number $\left(0 \leq r_{i} \leq 1\right)$ chosen from a uniform distribution, and $G\left(r_{i}\right)$ is an inversed cumulative Gaussian distribution with $\mu=0$ and $\sigma^{2}=2 D \Delta t$, where $D$ is the diffusion coefficient (note that the coefficient was set at 0.5 $\mu \mathrm{m}^{2} / \mathrm{s}$ ). Immobilization was derived from simple binding kinetics described by the effective on- and off-rates: $k_{o n} / k_{\text {off }}=F_{i m m} /\left(1-F_{i m m}\right)$, where $F_{i m m}$ is the relative number of immobile molecules (note that $k_{\text {on }}$ and $k_{\text {off }}$ were varied between 0.001 and 0.1 ). The probability for each particle to become immobilized (representing binding to chromatin) per unit time $(\Delta t)$ is then defined as $P_{\text {immobilise }}=k_{\text {on }}=k_{\text {off }} \cdot F_{i m m} /\left(1-F_{i m m}\right)$, where $k_{\text {off }}=1 / T_{\text {imm }}$, and $T_{\text {imm }}$ is the characteristic binding time. The probability to be released is given by $P_{\text {mobilise }}=k_{\text {off }}=1 / T_{i m m}$. Simulated FRAP curves were generated by counting the number of unbleached molecules in the bleached region after simulations of diffusion and binding during that time step.

\section{Fluorescence correlation spectroscopy (FCS)}

Fluorescence correlation spectroscopy (FCS) experiments were performed on a Zeiss LSM780 with a Plan Aprochromat 1.4NA 63x oil immersion lens, with $488 \mathrm{~nm}$ excitation laser and a 499-551 nm emission filter, on a GaSP array detector. Individual experiments were performed for 5 iterations of 10 seconds per cell at the same location. For every group at least 10 cells were measured. Because static bound protein does not give an autocorrelation, FCS is blind to static proteins. However, these proteins do bleach in the first iteration, showing up as a very slow moving molecule. For this reason the first iteration of every measurement was not taken along in the further analysis. The FCS volume was calibrated with a solution of Rhodamine $6 G$ to be able to convert the number of molecules in the FCS spot to a concentration. When cells are moving too much the FCS spot traverses different areas of the cell, or even ends up outside the cell, resulting in a disappearance of the autocorrelation. In such cases results were also removed. Data was globally analysed with the FFS data processor (SSTC, Belarus, http://www.sstcenter.com/) Next to the blinking GFP fraction two free diffusion coefficients were calculated. When no second coefficient was found only one coefficient was used.

Fluorescent in situ hybridization (FISH) 
WT and mutant ES cells were grown on slides coated with $0.2 \%$ gelatine. Once the right confluence was reached, ES cells were fixed with 3\% PFA (Sigma Aldrich P6148) in PBS $1 \mathrm{X}$ for $10 \mathrm{~min}$ at $4^{\circ} \mathrm{C}$. Cells were then permeabilized using $0.5 \%$ triton (Sigma Aldrich T8787) in PBS $1 \mathrm{X}$ for 6 min at $4^{\circ} \mathrm{C}$. Slides were stored in Ethanol $70 \%$ at $4^{\circ} \mathrm{C}$ until use in fluorescent in situ hybridizations (FISH) experiments.

The Xist Fosmid probe WI1-2363H9 was ordered from Chori BACPAC and maxi preparation was done using the BAC extraction kit from Macherey-Nagel (740436.25). The Xist Probe was directly labelled using nick translation kit (Abbott molecular 07J00-001) according to the manufacturer recommendation. Fluorescent dUTPs were purchased from Abbott molecular (Green :02N32-050, Red :02N34-050). FISH probe was further prepared by mixing $5 \mu$ of labelled probe, $3 \mu$ mouse Cot- 1 DNA (ThermoFisher 18440016), $1 \mu \mathrm{l}$ of salmon sperm DNA (ThermoFisher 15632011), sterile $\mathrm{H} 2 \mathrm{O}$ up to $50 \mu \mathrm{l}, 5 \mu \mathrm{l}$ Sodium Acetate $3 \mathrm{M} \mathrm{pH}=5.2$, and $125 \mu \mathrm{l}$ Ethanol $100 \%$ (Sigma Aldrich 24103 ) and precipitating the probe mix at $-20^{\circ} \mathrm{C}$ for $20 \mathrm{~min}$. Probe mix was then centrifuged at max speed for $15 \mathrm{~min}$ at $4^{\circ} \mathrm{C}$, and the pellet washed with ethanol $70 \%$. Pellet was air-dried and resuspended in $7 \mu$ of formamide $100 \%$ and denatured for $10 \mathrm{~min}$ at $75^{\circ} \mathrm{C}$ under agitation (900 rpm). Subsequently, $7 \mu$ of cold $2 \mathrm{X}$ hybridization buffer (4X SSC, $20 \%$ dextran sulfate, $2 \mathrm{mg} / \mathrm{ml} B S A$ ) were added to the denatured probe and the resultant mix was put on ice. In the mean time slides with ES cells were washed $3 X$ in PBS $1 X$, and then treated with RNAse A at $0.1 \mu \mathrm{g} / \mu \mathrm{l}$ ) in PBS 1X (ThermoFisher EN0531) for $30 \mathrm{~min}$ at $37^{\circ} \mathrm{C}$ in a humid chamber. Slides were rinsed in PBS 1X 3 times, then dehydrated in consecutive ethanol washes $(70 \%, 90 \%$ then $100 \%$ ), and air-dried at RT. Next, the probe was added to the slide and covered with a coverslip. Slides were denatured on a heating plate (Adamas SW85 slidewarmer) for $5 \mathrm{~min}$ at $75^{\circ} \mathrm{C}$. The plate was switched off and the slide left to cool down for $30 \mathrm{~min}$. Then the slide was put into a 50\%formamide/2X SSC chamber and incubated at $37^{\circ} \mathrm{C}$ overnight. The next day slides were washed in $50 \%$ formamide/2X SSC $(\mathrm{pH}=7.2-7.4)$ two times at $42^{\circ} \mathrm{C}$, one time in $2 X$ SCC at $42^{\circ} \mathrm{C}$, and then mounted with vectashild+DAPI (Vectorlabs $\mathrm{H}-1200$ ) and kept at $4^{\circ} \mathrm{C}$ protected from light. Slides were imaged using a fluorescent microscope (Axio Imager M2). Fluorescent spots were counted using Fiji (ImageJ) ${ }^{11}$.

RNA-sequencing and alignment. 
RNA sequencing (RNA-Seq) was performed using the Illumina HiSeq platform. Briefly, RNA-Seq samples were prepared with the Illumina TruSeq Stranded mRNA Library Prep Kit. The resulting DNA libraries were sequenced according to the Illumina TruSeq Rapid v2 protocol on an Illumina HiSeq2500 sequencer, generating single-end reads of $50 \mathrm{bp}$. Reads were aligned to the mouse genome (mm10 build) using HISAT2 ${ }^{12}$. Sample scaling and statistical analysis were performed using the $\mathrm{R}$ package $D E S e q 2^{13}$; genes with an adjusted $\mathrm{P}<0.05$ (Wald test) were considered differentially expressed. Standard RPKM values (or log2 transformed) were used as an absolute measure of gene expression. Genes with average RPKM $<1$ in both experimental groups compared were considered not expressed and excluded from downstream analysis. Pathway enrichment analysis on differentially expressed genes was performed using Metascape ${ }^{14}$. Published RNA-Seq fastq files were downloaded from the Gene Expression Omnibus (GSE123636 ( ref $\left.^{15}\right)$,GSE98671 (ref $\left.{ }^{16}\right)$ ) and aligned as described above using the Octopus-toolkit ${ }^{17}$.

\section{Chromatin immunoprecipitation followed by high-throughput sequencing}

Chromatin immunoprecipitation (ChIP) was performed as described ${ }^{6}$ using homemade polyclonal rabbit CTCF antibodies and rabbit pre-immune serum as control. Briefly, $40-80^{*} 10^{6}$ cells were harvested and cross-linked with $1 \%$ formaldehyde (Sigma) for 10 minutes at room temperature. Reactions were quenched with glycine (Sigma). Cells were treated first with cell lysis buffer (10 mM Tris pH 8.0, $10 \mathrm{mM} \mathrm{NaCl}$, $0.2 \%$ NP-40 (Sigma), Protease Inhibitor) and then with nuclear lysis buffer (50 mM Tris $\mathrm{pH}$ 8.0, $10 \mathrm{mM}$ EDTA, 1\% SDS, Protease Inhibitor). Sonication was performed with a bioruptor (Diagnode) to yield DNA fragments up to $800 \mathrm{bp}$. We performed CTCF immunoprecipitations with home-made polyclonal rabbit CTCF antibodies (N2.2, made using previously described protocols ${ }^{2}$ ) and used rabbit pre-immune serum as control.

A ChIP DNA library was prepared according to the Illumina protocol (www.illumina.com). Briefly, $10 \mathrm{ng}$ of end-repaired ChIPped DNA was ligated to adapters, size selected on gel ( $200 \pm 25$ bp range), and PCR amplified using Phusion polymerase as follows: $30 \mathrm{sec}$ at $98^{\circ} \mathrm{C}, 18 \mathrm{cycles}$ of $\left(10 \mathrm{sec}\right.$ at $98^{\circ} \mathrm{C}, 30 \mathrm{sec}$ at $65^{\circ} \mathrm{C}$, $30 \mathrm{sec}$ at $72^{\circ} \mathrm{C}$ ), $5 \mathrm{~min}$ at $72^{\circ} \mathrm{C}$ final extension. Cluster generation was performed using the Illumina Cluster Reagents preparation. The library was sequenced on the Illumina HiSeq2000 systems to generate 36 bp reads and a $7 \mathrm{bp}$ index read. 


\section{Computational analysis of ChIP-Seq datasets}

Samples were de-multiplexed and aligned against the mouse reference genome using NARWHAL and Bowtie alignment software ${ }^{18}$. In the first set of computational analysis of CTCF ChIP-Seq data, reads were aligned to the $\mathrm{mm} 9$ (and later $\mathrm{mm} 10$ ) reference genome using Bowtie $2^{19}$. After read extension, coverage vectors were produced and converted to bigwig format using an in-house processing pipeline. Visualization of coverage vectors was done with the IGV genome browser. Peaks were identified using PeakRanger with standard settings ${ }^{20}$. Here, peaks were identified based on read dense regions defined by $p$-value $<0.0001$. The negative (mock) control was used to subtract the background (FDR $<0.05$ and $>3$ fold enrichment between ChIP and mock). In order to obtain a robust set of peaks we used the presence of the core CTCF motif as criteria ${ }^{21}$. We used the top 1000 sites from each dataset and constructed a position specific scoring matrix (PSSM). This PSSM was used to perform a genomewide prediction of CTCF motifs across the complete mouse genome using the Patser tool $^{22}$ with a Patser score $>7$. Peak intervals detected with the GFP-CTCF dataset that overlapped with at least one instance of a core motif were used for subsequent analysis (22,216 sites).

In general, all downstream handling of reads, genomic intervals, features and related operations as well as sequence manipulation was done in $R$ using standard $R$ as well as specific R/BioConductor packages (mostly GenomicRanges, GenomicFeatures, ShortRead, rtracklayer). For the 22,216 CTCF binding regions found in the first computational analysis we calculated the number of reads for the individual zinc finger deletions as well as for the GFP-CTCF dataset. DESeq ${ }^{23}$ was used for proper normalization of the data as well as to determine the fold change between the individual zinc finger mutants and the GFP-CTCF dataset. Fold changes were log2-transformed and those sites with an absolute log2 (fold change) larger than 2 in at least one zinc finger mutant were further subjected to cluster analysis using kmeans in order to identify co-regulated CTCF binding sites.

To analyze the ZF-mediated recognition by CTCF all binding sites with a log2 (fold change) $<-2$ for an individual zinc finger deletion were identified and the corresponding sequences were extracted and collected in a $70 \mathrm{bp}$ window (using the BioConductor package BSgenome.Mmusculus.UCSC.mm9). Sequences were summarized as a weblogo using the $\mathrm{R}$ package webLogo. To analyze the distribution 
of CTCF over genes, the mm9 genome was partitioned into regions based on RefSeq annotations, TSS: $+/-1 \mathrm{~kb}$ around RefSeq TSSs, TSS upstream: -1 to $-10 \mathrm{~kb}$, exons, introns, TES: $+/-1 \mathrm{~kb}$, rest: intergenic. All intervals of a given feature class were taken together and the ratio relative to the complete genome size was calculated to obtain the genomic background distribution. RefSeq annotations for $\mathrm{mm} 9$ were used to calculate the distance for each of the $\sim 22,000$ CTCF core motifs to the next transcriptional start sites. Data is plotted as a histogram within a window of $+/-40 \mathrm{~kb}$ (or $50 \mathrm{~kb}$ ) around the TSS.

CTCF sites containing core and upstream motif (UC) were identified using the top 200 reduced sites identified in the GFP-CTCF-d9 dataset to construct a position specific scoring matrix (PSSM). This matrix was used to identify potential motif instances across the complete mm9 genome using Patser. Under the applied setting we identified about 1 million instances of the upstream and core motifs. Next, we identified the 22,216 CTCF core motifs from the UC sites and found 2718 cases were the upstream motif was located at a position $-18 \mathrm{bp}$ relative to the CTCF core motif. We determined the log2 (fold change) binding change for a given zinc finger mutant compared to all GFP-CTCF for those sites having an upstream motif at position -18 bp.

To analyze chromatin composition near UC sites we generated average cumulative plots, showing the binding of histones, histone modification marks and chromatin factors in the context of all CTCF sites and UC-containing CTCF sites. Data were downloaded from the Gene Expression Omnibus in short read archive format (sra): H3 (GSE23830), H3.3 (GSE16893), H3K27Ac (GSE24164) and H3K4me3 (GSE24165), H3K27me3 (GSE15519), H3K36me3 (GSE12241) and H3K9me3 (GSE12241), RNA Polll (ser5P, GSE20485) and SMC1 (GSE22557), OCT4 (GSE11431), and CTCFL (GSE34094). FASTQ data were extracted using SRA tools version 2.18. Reads were aligned to the $\mathrm{mm} 9$ genome as described above. After read extension coverage vectors were produced. These were used to collect binding data in a +/- $20 \mathrm{~kb}$ window with a $200 \mathrm{bp}$ step size around the CTCF binding sites.

For CTCF ChIP-Seq data integration with $\mathrm{Hi}-\mathrm{C}$ (see below), we parsed bam files to HOMER software ${ }^{24}$. CTCF peaks were assigned to their nearest gene using the annotatePeaks script. Overlapping and non-overlapping regions/peaks between two bed files were identified using HOMER mergePeaks (-d given option), requiring a minimal overlap of $1 \mathrm{bp}$. Histograms of ChIP-Sequencing signal were generated using 
the annotatePeaks script (-hist mode). Published ATAC-Seq ${ }^{25}$ and MNAse-Seq ${ }^{26}$ data were downloaded and aligned as described above using the Octopus-toolkit to generate bam files for downstream analysis with HOMER.

Methylated DNA sequencing (MeD-seq) sample preparation

LpnPI (New England Biolabs) digestions were carried out on DNA samples according to the manufacturer's protocol. Reactions contained $50 \mathrm{ng}$ in a $10 \mu \mathrm{L}$ volume and digestion took place overnight in the absence of enzyme activators. Digests of genomic DNA with LpnPI resulted in snippets of $32 \mathrm{bp}$ around the fully-methylated recognition site that contains $\mathrm{CpG}$. The DNA concentration was determined by the Quant-iT ${ }^{\mathrm{TM}}$ High-Sensitivity assay (Life Technologies; Q33120) and $50 \mathrm{ng}$ ds DNA was prepared using the ThruPlex DNA-seq 96D kit (Rubicon Genomics cat\#R400407). Stem-loop adapters were blunt end ligated to repaired input DNA and amplified ( $4+10$ cycles) to include dual indexed barcodes using a high fidelity polymerase to yield an indexed Illumina NGS library. Twenty microliters of amplified end product was purified on a Pippin HT system with 3\% agarose gel cassettes (Sage Science). Multiplexed samples were sequenced on Illumina HiSeq2500 systems for single read of 50 base pairs according to manufacturer's instructions. Dual indexed samples were demultiplexed using bcl2fastq software (Illumina).

\section{$M e D$-seq data processing}

Data processing was carried out using specifically created scripts in Python version 2.7.5. Raw fastq files were subjected to Illumina adaptor trimming and reads were filtered based on LpnPI restriction site occurrence between 13-17 bp from either 5' or 3 ' end of the read. Reads that passed the filter were mapped to mm10 using bowtie2.1.0. Multiple and unique mapped reads were used to assign read count scores to each individual $\mathrm{LpnPI}$ site in the mm10 genome. BAM files were generated using SAMtools for visualization. Gene and $\mathrm{CpG}$ island annotations were downloaded from UCSC (MM10). Genome wide individual LpnPI site scores were used to generate read count scores for the following annotated regions: transcription start site (TSS) (1 kb before and $1 \mathrm{~kb}$ after), CpG islands and genebody (1 kb after TSS till TES).

$M e D$-seq data analysis 
Data analysis was carried out in Python 2.7.5. DMR detection was performed between two datasets containing the regions of interest (TSS, genebody or $\mathrm{CpG}$ islands) using the Chi-Squared test on read counts. Significance was called by either Bonferroni or FDR using the Benjamini-Hochberg procedure. Differently methylated regions were used for unsupervised hierarchical clustering, the Z-score of the read counts was used for normalization and is also shown in the heatmaps. In addition a genome wide sliding window was used to detect sequentially differentially methylated LpnPI sites. Statistical significance was called between LpnPI sites in predetermined groups using the Chi-squared test. Neighboring significantly called LpnPI sites were binned and reported, DMR threshold was set at a minimum of ten LpnPI sites, a minimum size of $100 \mathrm{bp}$ and a 2 -fold change in read counts.

\section{In-situ Hi-C}

In-situ Hi-C libraries were generated as previously described ${ }^{25,27}$. Two-to-three million ES cells were used as starting material for chromatin digestion using $100 \mathrm{U}$ Mbol (New England Biolabs) for 2 hours, followed by addition of another $100 \mathrm{U}$ (2 hour incubation) and a final $100 \mathrm{U}$ before overnight incubation. After digestion, nuclei were pelleted, resuspended in fresh $1 \times$ NEB2 buffer and restriction fill-in with bio-dATP. Ligation was performed overnight at $24^{\circ} \mathrm{C}$ using 10,000 cohesive end units per reaction; 5) decrosslinked and purified DNA was sonicated to an average size of 300-400 bp using a Bioruptor Pico (Diagenode; 7 cycles of $20 \mathrm{~s}$ on and $60 \mathrm{~s}$ off); 6) DNA fragment size selection was only performed after final library amplification; 7) library preparation was performed with the NEBNext DNA Library Prep Kit (New England Biolabs) using $3 \mu \mathrm{L}$ NEBNext adaptor in the ligation step; 8) libraries were amplified for 8-12 cycles using Herculase II Fusion DNA Polymerase (Agilent) and purified/size-selected using Agencourt AMPure XP beads (>200 bp). Hi-C Library quality was assessed lowcoverage sequencing, after which every biological replicate $(n=3)$ was sequenced at high-coverage on an Illumina NextSeq500. Data from biological replicates were pooled for downstream analysis. We sequenced 370 million reads in total to obtain 100 million valid interactions per condition.

Computational analysis of $\mathrm{Hi}-\mathrm{C}$ datasets.

$\mathrm{Hi}-\mathrm{C}$ data analysis was conducted as previously described ${ }^{25}$. Read-pairs were mapped to the reference genome $(\mathrm{mm} 10)$ using TADbit ${ }^{28}$. The TADbit filtering module 
was used to remove non-informative contacts (e.g. self-circles, dangling-ends and duplicates). Low quality bins (those presenting low contacts numbers) were removed as implemented in TADbit's "filter_columns" routine. A single round of ICE normalization ${ }^{29}$ ("vanilla" normalization ${ }^{27}$ ) was performed and contact matrices were corrected to achieve an average content of one interaction per cell. Reproducibility scores were calculated using the spectral decomposition method from the HiC-spector package $^{30}$.

Identification of subnuclear compartments and topologically associated domains (TADs).

To segment the genome into $\mathrm{A} / \mathrm{B}$ compartments, normalized $\mathrm{Hi}-\mathrm{C}$ matrices at $100 \mathrm{~kb}$ resolution were corrected for decay as previously published, grouping diagonals when signal-to-noise was below 0.0511. Corrected matrices were the split into chromosomal matrices and transformed into correlation matrices using the Pearson product-moment correlation. The first component of a PCA (PC1) on each of these matrices was used as a quantitative measure of compartmentalization and AT content was used to assign negative and positive PC1 categories to the correct compartments. If necessary, the sign of the PC1 (which is randomly assigned) was inverted so that positive PC1 values corresponded to A compartment regions and vice versa for the B compartment.

Normalized contact matrices at $50 \mathrm{~kb}$ resolution were used to define TADs, using a previously described method with default parameters ${ }^{31}$. First, for each bin, an insulation score was obtained based on the number of contacts between bins on each side of a given bin, and borders were called searching for minima within the insulation score.

Meta-analysis of borders, loops and interactions between super enhancers (SE) To assess whether particular parts of the $\mathrm{Hi}-\mathrm{C}$ interaction matrices had common structural features, we performed meta-analyses by merging individual sub-matrices into an average meta-matrix in a similar fashion as previously published. Three types of meta-analysis were performed. First, to study the behavior of TAD borders all TADs of sizes ranging from 0.5 to $1.5 \mathrm{Mb}$ were selected. Then we defined a flanking region of $1 \mathrm{Mb}$ around the border and gathered the observed and expected (by distance decay) matrix counts. Setting up their relative position to the corresponding border, the matrices were stacked to obtain a meta-contact matrix around TAD borders for 
each condition. This information was summarized by comparing the average $\log _{2}$ fold change of contact enrichment between bins inside and outside TADs. Second, using $5 \mathrm{~kb}$ resolution contact maps normalized for sequencing depth and genomic biases using One $\mathrm{D}^{32}$, we investigated the dynamics of a previously identified set of chromatin loops in primary $\mathrm{B}$ cells and $\mathrm{PSCs}^{33}$ by extracting interaction counts $100 \mathrm{~kb}$ up and downstream of loop anchor regions. Meta-loop matrices were then calculated by averaging individually subtracted loop matrices into a single one per group. Third, we studied whether SE are likely to find each other more than expected within a genomic distance ranging from 5 to $10 \mathrm{Mb}$. $\mathrm{Hi}-\mathrm{C}$ matrices were generated at $10-\mathrm{kb}$ resolution using HiCExplorer as described ${ }^{34}$ and long range interactions (5-10 Mb) between SE were computed using the HiCExplorer tool hicAggregateContacts.

\section{De novo loop calling}

$\mathrm{Hi}-\mathrm{C}$ matrices in cool format were used to call loops with Mustache software ${ }^{35}$ at 10 $\mathrm{kb}$ resolution and a P-value threshold of 0.1 . We used coolpup.py ${ }^{36}$ to pile-up normalized $\mathrm{Hi}-\mathrm{C}$ signals at loops identified and generate meta-plots.

\section{Virtual 4C analysis}

$\mathrm{Hi}-\mathrm{C}$ matrices for virtual 4C profiles were further smoothed using a focal (moving window) average of one bin. The profiles were generated from these normalized matrices and correspond to histogram representation of the lines of the matrices containing the baits (therefore expressed as counts per hundred of normalized reads within the region depicted). 


\section{References}

1. Sleutels, F. et al. The male germ cell gene regulator CTCFL is functionally different from CTCF and binds CTCF-like consensus sites in a nucleosome composition-dependent manner. Epigenetics Chromatin 5, 8 (2012).

2. Heath, H. et al. CTCF regulates cell cycle progression of alphabeta T cells in the thymus. Embo J 27, 2839-50 (2008).

3. Chuma, S. \& Nakatsuji, N. Autonomous transition into meiosis of mouse fetal germ cells in vitro and its inhibition by gp130-mediated signaling. Dev Biol 229, 468-79 (2001).

4. Dragestein, K.A. et al. Dynamic behavior of GFP-CLIP-170 reveals fast protein turnover on microtubule plus ends. J Cell Biol 180, 729-37 (2008).

5. Lukinavicius, G. et al. SiR-Hoechst is a far-red DNA stain for live-cell nanoscopy. Nat Commun 6, 8497 (2015).

6. van de Nobelen, S. et al. CTCF regulates the local epigenetic state of ribosomal DNA repeats. Epigenetics Chromatin 3, 19 (2010).

7. Leslie, K. \& Galjart, N. Going solo: measuring the motions of microtubules with an in vitro assay for TIRF microscopy. Methods Cell Biol 115, 109-24 (2013).

8. Pagliara, S. et al. Auxetic nuclei in embryonic stem cells exiting pluripotency. Nat Mater 13, 638-644 (2014).

9. Cattoglio, C. et al. Determining cellular CTCF and cohesin abundances to constrain 3D genome models. BioRxiv (2018).

10. Geverts, B., van Royen, M.E. \& Houtsmuller, A.B. Analysis of biomolecular dynamics by FRAP and computer simulation. Methods Mol Biol 1251, 109-33 (2015).

11. Schneider, C.A., Rasband, W.S. \& Eliceiri, K.W. NIH Image to ImageJ: 25 years of image analysis. Nat Methods 9, 671-5 (2012).

12. Kim, D., Langmead, B. \& Salzberg, S.L. HISAT: a fast spliced aligner with low memory requirements. Nat Methods 12, 357-60 (2015).

13. Love, M.I., Huber, W. \& Anders, S. Moderated estimation of fold change and dispersion for RNA-seq data with DESeq2. Genome Biol 15, 550 (2014).

14. Zhou, Y. et al. Metascape provides a biologist-oriented resource for the analysis of systems-level datasets. Nat Commun 10, 1523 (2019).

15. Hansen, A.S. et al. Distinct Classes of Chromatin Loops Revealed by Deletion of an RNA-Binding Region in CTCF. Mol Cell (2019).

16. Nora, E.P. et al. Targeted Degradation of CTCF Decouples Local Insulation of Chromosome Domains from Genomic Compartmentalization. Cell 169, 930-944 e22 (2017).

17. Kim, T., Seo, H.D., Hennighausen, L., Lee, D. \& Kang, K. Octopus-toolkit: a workflow to automate mining of public epigenomic and transcriptomic next-generation sequencing data. Nucleic Acids Res 46, e53 (2018).

18. Brouwer, R.W., van den Hout, M.C., Grosveld, F.G. \& van ljcken, W.F. NARWHAL, a primary analysis pipeline for NGS data. Bioinformatics 28, 284-5 (2012).

19. Langmead, B. \& Salzberg, S.L. Fast gapped-read alignment with Bowtie 2. Nat Methods 9, 357-9 (2012).

20. Feng, X., Grossman, R. \& Stein, L. PeakRanger: a cloud-enabled peak caller for ChIPseq data. BMC Bioinformatics 12, 139 (2011). 
21. Kim, T.H. et al. Analysis of the vertebrate insulator protein CTCF-binding sites in the human genome. Cell 128, 1231-45 (2007).

22. Thomas-Chollier, M. et al. RSAT: regulatory sequence analysis tools. Nucleic Acids Res 36, W119-27 (2008).

23. Anders, S. \& Huber, W. Differential expression analysis for sequence count data. Genome Biol 11, R106 (2010).

24. Heinz, S. et al. Simple combinations of lineage-determining transcription factors prime cis-regulatory elements required for macrophage and B cell identities. Mol Cell 38, 576-89 (2010).

25. Stadhouders, R. et al. Transcription factors orchestrate dynamic interplay between genome topology and gene regulation during cell reprogramming. Nat Genet 50, 238249 (2018).

26. Teif, V.B. et al. Genome-wide nucleosome positioning during embryonic stem cell development. Nat Struct Mol Biol 19, 1185-92 (2012).

27. Rao, S.S. et al. A 3D map of the human genome at kilobase resolution reveals principles of chromatin looping. Cell 159, 1665-80 (2014).

28. Serra, F. et al. Automatic analysis and 3D-modelling of Hi-C data using TADbit reveals structural features of the fly chromatin colors. PLoS Comput Biol 13, e1005665 (2017).

29. Imakaev, M. et al. Iterative correction of $\mathrm{Hi}-\mathrm{C}$ data reveals hallmarks of chromosome organization. Nat Methods 9, 999-1003 (2012).

30. Yan, K.K., Yardimci, G.G., Yan, C., Noble, W.S. \& Gerstein, M. HiC-spector: a matrix library for spectral and reproducibility analysis of Hi-C contact maps. Bioinformatics 33, 2199-2201 (2017).

31. Crane, E. et al. Condensin-driven remodelling of $\mathrm{X}$ chromosome topology during dosage compensation. Nature 523, 240-4 (2015).

32. Vidal, E. et al. OneD: increasing reproducibility of Hi-C samples with abnormal karyotypes. Nucleic Acids Res 46, e49 (2018).

33. Krijger, P.H. et al. Cell-of-Origin-Specific 3D Genome Structure Acquired during Somatic Cell Reprogramming. Cell Stem Cell 18, 597-610 (2016).

34. Ramirez, F. et al. High-resolution TADs reveal DNA sequences underlying genome organization in flies. Nat Commun 9, 189 (2018).

35. Roayaei Ardakany, A., Gezer, H.T., Lonardi, S. \& Ay, F. Mustache: multi-scale detection of chromatin loops from $\mathrm{Hi}-\mathrm{C}$ and Micro-C maps using scale-space representation. Genome Biol 21, 256 (2020).

36. Flyamer, I.M., Illingworth, R.S. \& Bickmore, W.A. Coolpup.py: versatile pile-up analysis of Hi-C data. Bioinformatics 36, 2980-2985 (2020). 